\title{
Photocathode Research for Electron Accelerators $\mathrm{PhD}$ Dissertation
}

\author{
James McCarter' \\ Department of Materials Science and Engineering \\ School of Engineering and Applied Science \\ University of Virginia, Charlottesville, VA 22903, USA
}

1jlm2ar@virginia.edu 


\section{Photocathode Research for Electron Accelerators}

A Dissertation
Presented to
the faculty of the School of Engineering and Applied Science
University of Virginia
in partial fulfillment
of the requirements for the degree
Doctor of Philosophy
by
James McCarter
December


APPROVAL SHEET

The dissertation

is submitted in partial fulfillment of the requirements

for the degree of

Doctor of Philosophy

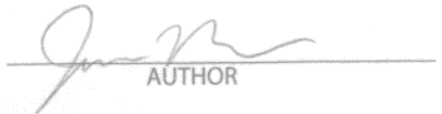

The dissertation has been read and approved by the examining committee:

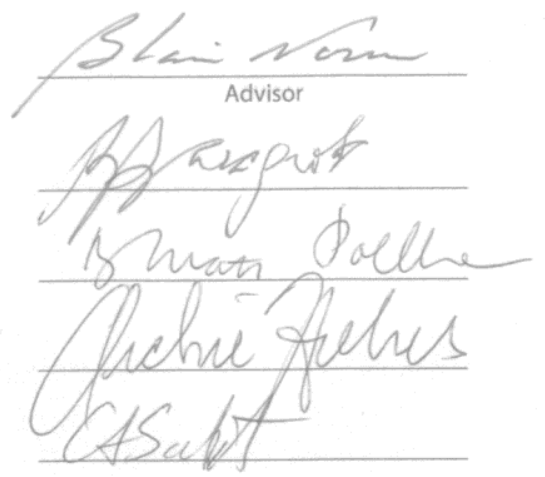

Accepted for the School of Engineering and Applied Science:

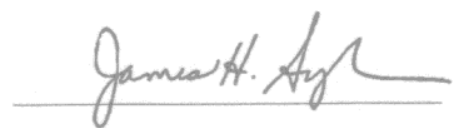

Dean, School of Engineering and Applied Science

December 


\section{Contents}

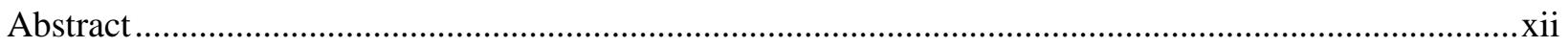

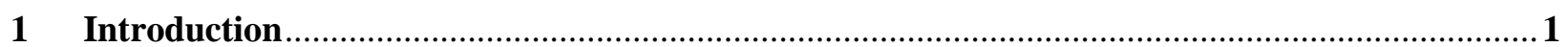

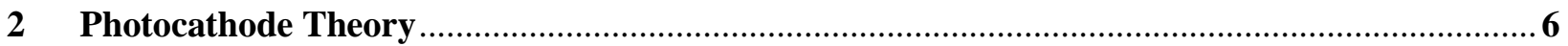

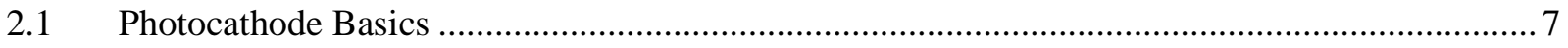

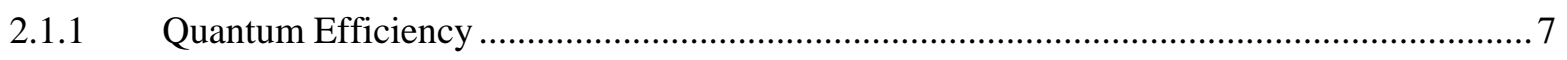

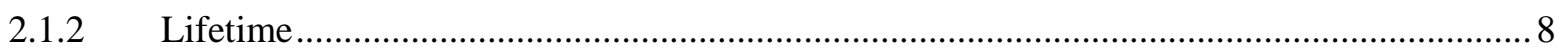

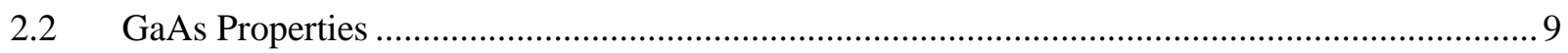

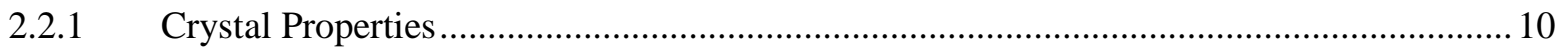

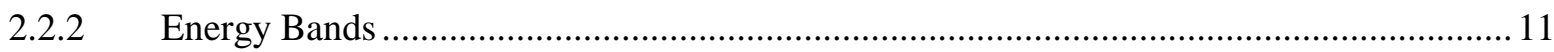

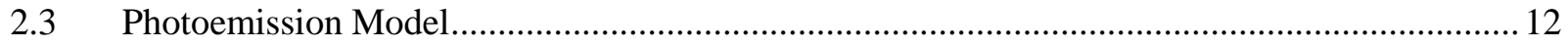

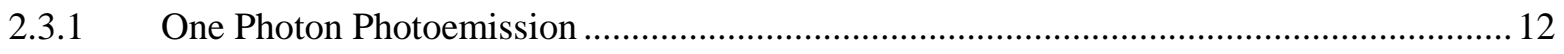

2.3.2 Two Photon Photoemission ........................................................................................ 15

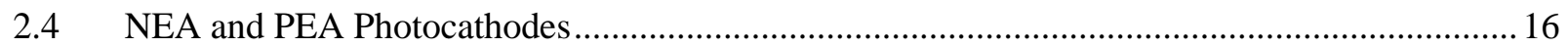

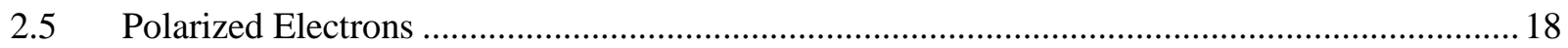

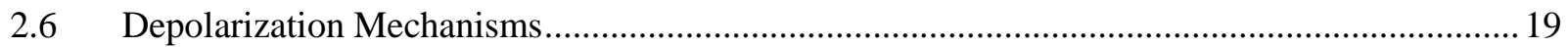

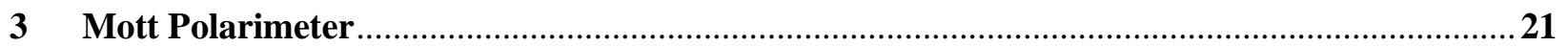

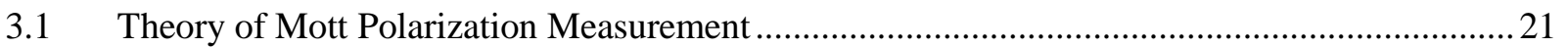

3.2 MicroMott Polarimeter Design and Techniques ....................................................................2 24

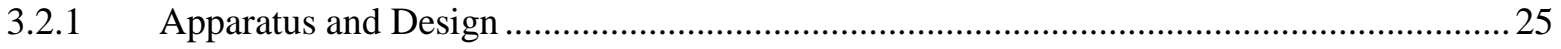

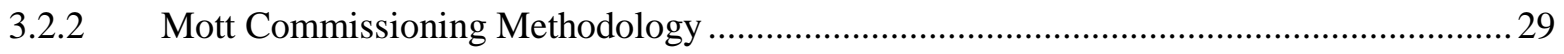

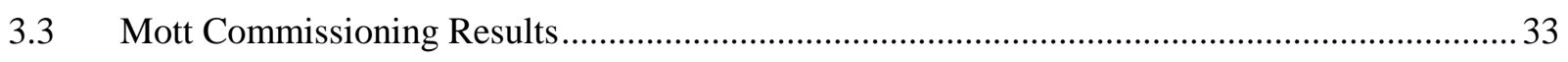

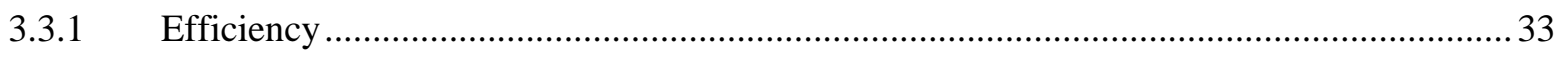

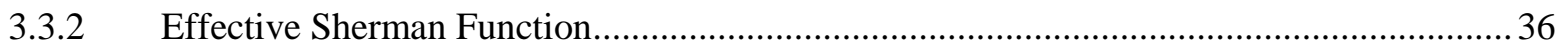

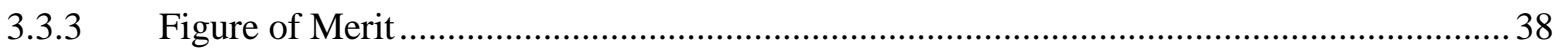

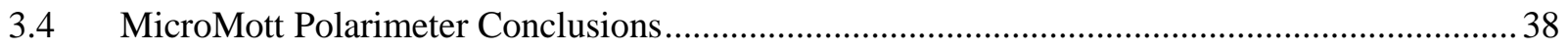

4 GaAs: Polarization from Two-Photon Photoemission............................................................. 40

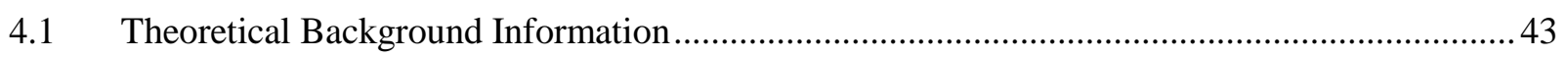


4.1.1 Generation of Photoelectrons by One- and Two-Photon Absorption ............................... 43

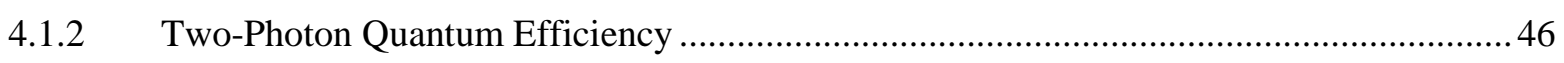

4.1.3 Electron Polarization via One- and Two- Photon Absorption ......................................... 48

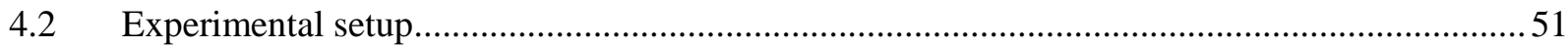

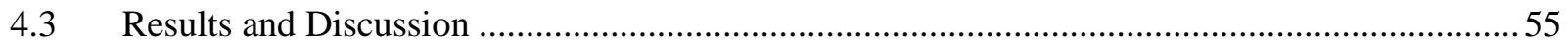

4.3.1 One- and Two-Photon Quantum Efficiency …..............................................................5

4.3.2 Electron Polarization from Thick $(625 \mu \mathrm{m})$ Unstrained GaAs .......................................60

4.3.3 Electron Polarization from Thin Unstrained GaAs .......................................................... 62

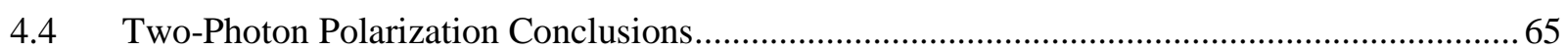

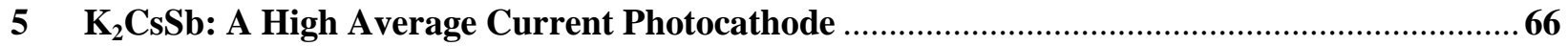

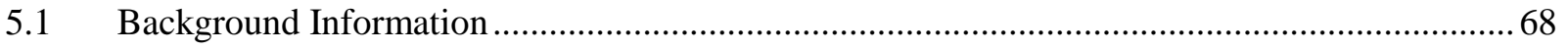

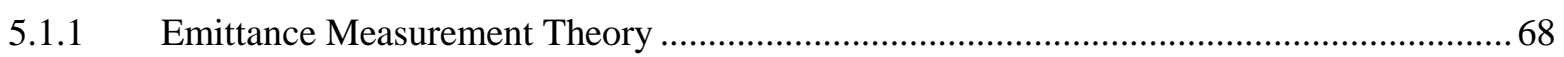

5.1.2 GaAs Photocathode QE Decay Mechanisms ..................................................................... 72

$5.2 \quad \mathrm{~K}_{2} \mathrm{CsSb}$ Photocathode Experimental Methodology .............................................................. 75

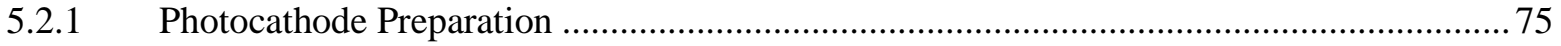

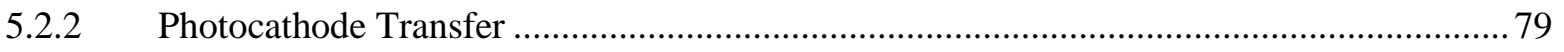

5.2.3 Jefferson Lab Photogun Testing Equipment ................................................................ 82

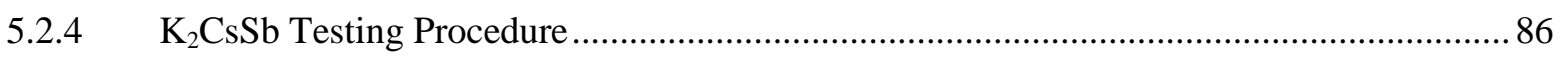

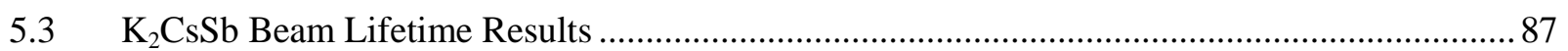

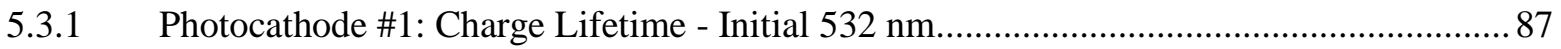

5.3.2 Photocathode \#1: Charge Lifetime - $440 \mathrm{~nm}$ and Repeated $532 \mathrm{~nm}$.............................90

5.3.3 Photocathode \#1: Laser Heating ……........................................................................... 94

5.3.4 Photocathode \#2: Ion Bombardment - Biased and Grounded Anode ................................95

5.3.5 Photocathode \#2: Laser Heating …............................................................................ 98

5.3.6 Photocathode \#2: Emittance ................................................................................... 99

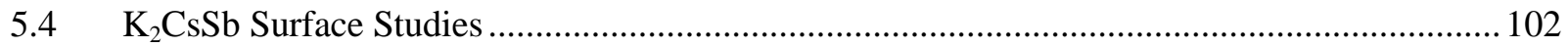

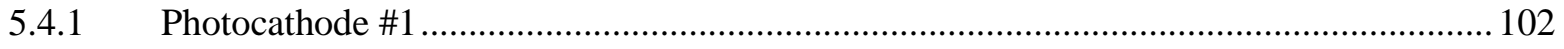

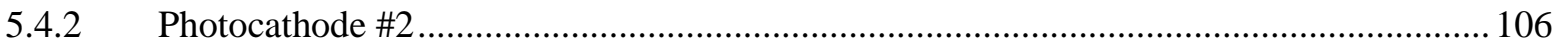

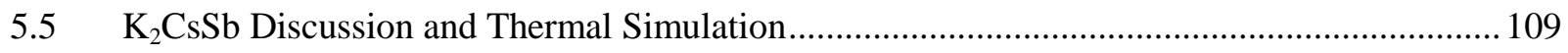

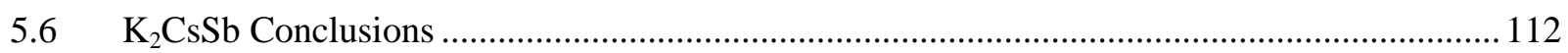

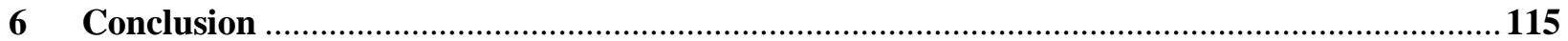

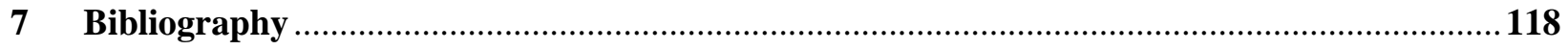




\section{List of Tables}

Table 3.1: Comparison of various mini-Mott and micro-Mott designs at $20 \mathrm{kV}$ target bias

Table 4.1: Photoemitted electron polarization taken for one- and two-photon absorption for three different samples of GaAs. .

Table 4.2: GaAs material properties as related to one- and two- photon polarized emission. .64

Table 5.1: Maximum simulated temperature at the illuminated location for a given absorbed laser power for a $\mathrm{K}_{2} \mathrm{CsSb}$, stainless steel, and aluminum puck, as well as a $\mathrm{K}_{2} \mathrm{CsSb}$, molybdenum puck. ...... 112 


\section{List of Figures}

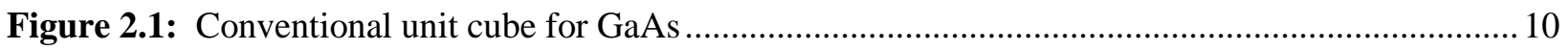

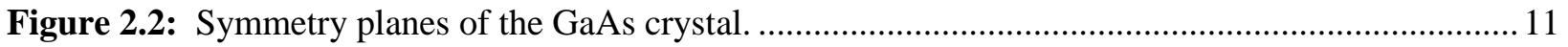

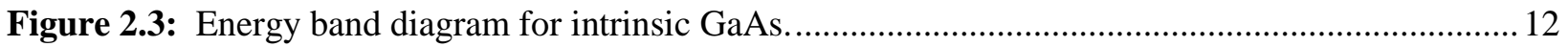

Figure 2.4: Spicer's Three-Step Photoemission Process …................................................................. 13

Figure 2.5: Optical absorption coefficients for some NEA semiconductors.......................................... 14

Figure 2.6: Formation of a NEA surface. p-doping GaAs lower the semiconductor vacuum level via band

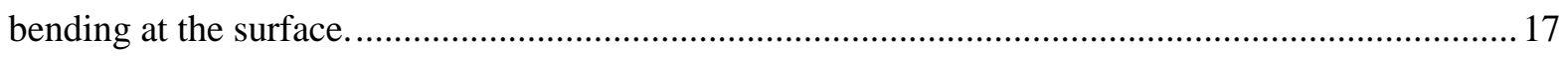

Figure 2.7: Photoemission from PEA and NEA GaAs compared in three different spaces ..................... 18

Figure 2.8: Optical transitions in GaAs with circularly polarized light ............................................... 19

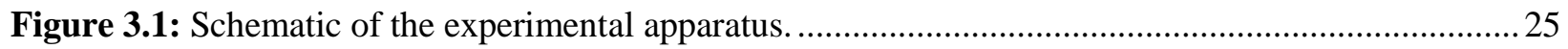

Figure 3.2: SIMION model of beam transport through the polarized electron source ............................22

Figure 3.3: a) Scale cross section drawing of polarimeter ...................................................................29

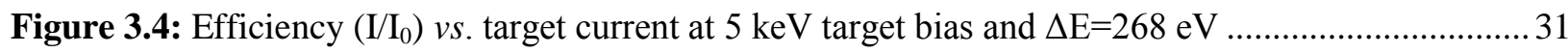

Figure 3.5: Asymmetry and CEM count rate as a function of discriminator threshold ........................... 32

Figure 3.6: Mott efficiency and CEM count rate as a function of Mott target current. ........................... 33

Figure 3.7: Variation as a function of target bias of the a) efficiency, $I / I_{0}$ for $\Delta E=268 \mathrm{eV}$; b) effective Sherman function for $\Delta \mathrm{E}=268 \mathrm{eV}(\circ)$ and extrapolated to $\Delta \mathrm{E}=0 \mathrm{eV}(\bullet)$; and c) the figure of merit, $\eta$, for $\Delta \mathrm{E}=268 \mathrm{eV}$. 34

Figure 3.8: Variation, as a function $\Delta \mathrm{E}$, of a) efficiency, $\mathrm{I} / \mathrm{I}_{\mathrm{o}}$; b) effective Sherman function, $\mathrm{S}_{\text {eff }}$, with weighted linear fit for extrapolation to $\Delta \mathrm{E}=0 \mathrm{eV}$; and c) figure of merit, $\eta$. Target bias of $20 \mathrm{kV}(\bullet)$;

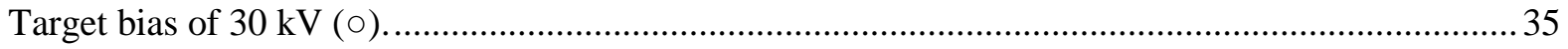

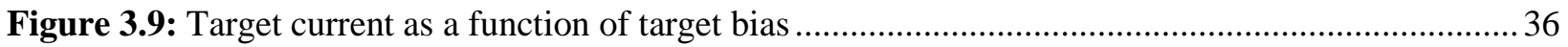


Figure 3.10: Measured electron polarization vs. target bias. .38

Figure 4.1: Various means to populate the conduction band of GaAs with circularly-polarized light..... 41

Figure 4.2: Schematic of the 1560nm light source .52

Figure 4.3: Representative QE vs. input power .57

Figure 4.4: Representative QE vs. peak power. .58

Figure 4.5: QE vs. peak intensity of the laser beam .59

Figure 4.6: Coefficient $\boldsymbol{\beta}$ of two-photon absorption as a function of the laser peak intensity.... 60

Figure 4.7: Polarization of the photoelectron beam from bulk GaAs at $778 \mathrm{~nm}$ and at $1560 \mathrm{~nm}$ as a function of the orientation of the quarter wave plate.

Figure 5.1: Diagram of ion backbombardment of a photocathode .73

Figure 5.2: QE map of a GaAs photocathode, showing characteristic QE decay due to ion bombardment over a period of many weeks.

Figure 5.3: Two views of the BNL deposition system used for fabrication of $\mathrm{K}_{2} \mathrm{CsSb}$ photocathodes..... 76

Figure 5.4: The transfer arm of the $\mathrm{BNL} \mathrm{K}_{2} \mathrm{CsSb}$ deposition system holding the stainless steel and aluminum puck. .77

Figure 5.5: Spectral response of the $\mathrm{K}_{2} \mathrm{CsSb}$ photocathodes created at BNL ...................................... 79

Figure 5.6: Schematic of the vacuum suitcase...... 80

Figure 5.7: The JLab vacuum suitcase attached to the BNL deposition chamber. 81

Figure 5.8: Photo of the photocathode puck transfer between the BNL deposition chamber and the JLab suitcase..

Figure 5.9: Schematic and photo of the photogun and beamline at JLAB's ITS..... 83

Figure 5.10: Photograph of the photogun and preparation chamber of the ITS, with the transfer suitcase attached. 84

Figure 5.11: Schematic of the DC high voltage photogun at JLab, with inverted ceramic insulator......... 85

Figure 5.12: QE maps of $\mathrm{K}_{2} \mathrm{CsSb}$ photocathode \#1 using $532 \mathrm{~nm}$ light. .89 
Figure 5.13: Charge lifetime of $\mathrm{K}_{2} \mathrm{CsSb}$ photocathode \#1 and GaAs:Cs versus the radial position of the laser spot relative to the electrostatic center of the cathode. .89

Figure 5.14: Photos of the beamline flange and its mu-metal shielding. 91

Figure 5.15: QE scans following the vacuum event 91

Figure 5.16: $Q E$ evolution during beam delivery from $\mathrm{K}_{2} \mathrm{CsSb}$ photocathode \#1 using $440 \mathrm{~nm}$ light and $532 \mathrm{~nm}$ light. 93

Figure 5.17: Beam current versus time using $\mathrm{K}_{2} \mathrm{CsSb}$ photocathode \#1 and $532 \mathrm{~nm}$ light with laser spot diameter: $500 \mu \mathrm{m}$ FWHM and $850 \mu \mathrm{m}$ FWHM .93

Figure 5.18: QE difference scans of $\mathrm{K}_{2} \mathrm{CsSb}$ photocathode \#1 using $440 \mathrm{~nm}$ laser at $200 \mathrm{~mW}$ for $12 \mathrm{hrs}$ and $532 \mathrm{~nm}$ laser at $700 \mathrm{~mW}$ for $2 \mathrm{hrs}$. .95

Figure 5.19: GaAs photocathode charge lifetimes for alternately biased and grounded anode. .97

Figure 5.20: The $\mathrm{QE}$ at the electrostatic center of $\mathrm{K}_{2} \mathrm{CsSb}$ photocathode $\# 2$ versus time at $10 \mathrm{~mA}$ average current and with the anode biased and grounded 97

Figure 5.20: $Q E$ evolution at $1 \mathrm{~mA}$ and $10 \mathrm{~mA}$ equivalent laser power. .99

Figure 5.21: Example of beam widths measured by a three-wire harp scanned across the beam path and Gaussian beam widths in the $\mathrm{x}$ direction for different focusing solenoid field strengths, for beam extracted from the electrostatic center of photocathode \#2. 100

Figure 5.22: QE scan of photocathode \#1 prior to transfer to the SEM and photograph of the entire photocathode showing the locations evaluated with SEM and EDS...... 103

Figure 5.23: Photographs of photocathode \#1 after removal from the SEM, showing the central damaged area and the transition away from the damage area..... 104

Figure 5.24: (a) Surface of photocathode \#1 at 400x magnification and (b) 3000x magnification. 105

Figure 5.25: Chemical assay relative elemental species contribution by percent of total, as taken by EDS from SEM 400x images of locations 1-4 from photocathode \#1. 105

Figure 5.26: QE scan and photograph of photocathode \#2 prior to transfer to the SEM. 106 
Figure 5.27: $\mathrm{SEM}$ images at 3000x magnification using an $8 \mathrm{keV}$ electron beam at photocathode locations that had been illuminated with $2 \mathrm{~W}$ laser light, in one case while extracting beam at $10 \mathrm{~mA}$ using a laser spot $850 \mu \mathrm{m}$ FWHM and the other case heating the photocathode with a laser spot 500 $\mu \mathrm{m}$ FWHM without generating beam. 108

Figure 5.28: Chemical assay relative elemental species contribution by percent of total, as taken by EDS from SEM 400x from photocathode \#2. 108

Figure 5.29: Schematic view of the elements used in the ANSYS 14.0 thermal analysis and close-up cross sectional view of the photocathode and the stainless steel/aluminum puck with $1.3 \mathrm{~W}$ of laser light distributed over a $0.5 \mathrm{~mm}$ diameter region. 110 


\section{Acknowledgements}

I would like to thank my advisors Blaine Norum and Matt Poelker for their guidance and support. Thanks to Timothy Gay, Marcy Stutzman, Riad Suleiman and Russell Mammei for teaching me so much about the field and passing on at least a small part of their vast knowledge. Thanks to Andrei Afanasev and Ara Kechiantz for their theoretical expertise. Thank you to Phil Adderley, Jim Clark, John Hanskneckt, and Steve Covert for all of their technical expertise. Also, thanks to Vickie Thomas, Kimberly FitzhughHiggins, Dawn Shifflet, and Tammie Shifflet for invaluable administrative support. Thanks to my parents

for giving me the tools to succeed. Finally, thanks to my lovely wife Sara for her incredible patience, understanding, and love. I could not have done this without you. 


\section{Abstract}

This dissertation presents studies of two different types of photocathodes used at electron accelerators: $\mathrm{GaAs}$ and $\mathrm{K}_{2} \mathrm{CsSb}$. The spin polarization of photoelectrons extracted from GaAs photocathodes was evaluated using traditional one-photon absorption and two-photon absorption, to determine the validity of speculation that two-photon absorption would provide significantly higher polarization. To accomplish this study, a novel compact, retarding-field Mott polarimeter was designed, built and commissioned. The second type of photocathode, $\mathrm{K}_{2} \mathrm{CsSb}$, was manufactured at Brookhaven National Laboratory and transported to Jefferson Lab in an ultrahigh vacuum "suitcase" and installed within a DC high voltage gun. Charge lifetime measurements were made using a $\mathrm{K}_{2} \mathrm{CsSb}$ photocathode and compared to those of GaAs under identical operating conditions. The surface morphology of the used $\mathrm{K}_{2} \mathrm{CsSb}$ was also studied.

Many important atomic, nuclear and high energy physics experiments rely on using highly spin-polarized electron beams obtained via photoemission from GaAs photocathodes. Remarkably, even after 30 years of GaAs photogun operation, there are still some lingering questions associated with the beam polarization values being lower than expected. Bulk GaAs can theoretically provide $50 \%$ polarization but typically provides $\sim 35 \%$. Reduced polarization is attributed to a number of proposed depolarization mechanisms. Recently, it was proposed that two-photon absorption could be exploited to selectively populate the conduction band with electrons of just one spin state providing $100 \%$ polarization. These claims were refuted by others on theoretical grounds. In this thesis, for the first time, two-photon photoemission was demonstrated with beam delivered to a novel polarimeter constructed for this experiment. Polarization was found to be comparable to that obtained with conventional one-photon absorption, and to converge to the same value with a reduction in thickness of the active layer, thereby providing additional experimental insight to the polarized photoemission process an depolarization mechanisms in GaAs.

Many proposed accelerators (light sources, energy recovery linacs and electron cooling machines) require extremely high average beam current $(\sim 100 \mathrm{~mA})$. The highest average current accelerator today, the FEL at JLab, can operate at up to $10 \mathrm{~mA}$ and relies on photoemission from GaAs, even though spin polarization is not required. GaAs is an extremely delicate material, prone to failure and requiring ultrahigh vacuum conditions. In the early $1990 \mathrm{~s}$, the multi-alkali photocathode $\mathrm{K}_{2} \mathrm{CsSb}$ was used to demonstrate $32 \mathrm{~mA}$ average current inside an RF gun with poor vacuum characteristics. This experiment lasted just minutes and was terminated shortly after the high current milestone was met. Based on this early work, many people believe $\mathrm{K}_{2} \mathrm{CsSb}$ represents a much better photocathode choice for unpolarized accelerator applications, providing high current for long periods of time and under less stringent operating conditions, but a detailed comparative study using the same apparatus has not been done. To address the high current reliability issue, two $\mathrm{K}_{2} \mathrm{CsSb}$ photocathodes were manufactured at Brookhaven National Lab and transported to Jefferson Lab via an ultrahigh vacuum transport chamber. They were installed within a $200 \mathrm{kV}$ DC high voltage photogun. Systematic charge lifetime measurements were performed using both photocathode materials under identical conditions. The performance of $\mathrm{K}_{2} \mathrm{CsSb}$ was shown to be highly dependent on extracted current. By combining this decay behavior with surface science measurements of

the used photocathodes, it was shown that heat quickly disrupts the capability of the $\mathrm{K}_{2} \mathrm{CsSb}$ photocathode. 


\section{Introduction}

Electron beams are accelerated to GeV energy at the Continuous Electron Beam Accelerating Facility (CEBAF) at Thomas Jefferson National Laboratory Facility (JLab), and are used to probe hadronic structure. Many experiments require a spin-polarized electron beam, where the spin axes of the electrons are aligned in a preferential direction. By flipping the direction of the electron spin, experimenters measure asymmetries in the output of decay products of electron collisions with targets of many varieties. The production of these decay events is dependent on the luminosity of the electron beam at the interaction point and is proportional to $\mathrm{IP}^{2}$, where $\mathrm{I}$ is the electron beam current, and $\mathrm{P}$ is its average longitudinal polarization, which is defined as:

$$
P=\frac{N_{+}-N_{-}}{N_{+}+N_{-}}
$$

where $\mathrm{N}_{+}$and $\mathrm{N}_{-}$are the number of electrons that, when measured with regard to the beam axis, have spins of $+\hbar / 2$ and $-\hbar / 2$, respectively [1.1]. To meet the demands of the scheduled physics program, the photoguns used to generate the electron beam must provide high current, high polarization and exhibit high reliability. Beamtime is oversubscribed at CEBAF, and improvements in the quality of the photogun could alleviate this problem.

The Center for Injectors and Sources (CIS) at JLab is tasked with supplying the accelerator and the experimental Halls with the highest quality electron beam possible, and to this end, actively works to improve electron sources. CIS operates test stands that allow for study of electron gun components, including sources, in a setting similar to the actual CEBAF injector. One apparatus is a low energy electron source attached to a micro-Mott polarimeter, which allows for the measurement of polarization 
CHAPTER 1: INTRODUCTION

of electron beams extracted from different materials. Another apparatus consists of a $100 \mathrm{kV}$ DC high voltage gun attached to an ultrahigh vacuum beamline. These test stands, and a $\mathrm{K}_{2} \mathrm{CsSb}$ deposition chamber at BNL, where used to conduct the research of this dissertation and are described below.

GaAs, and other semiconductors in the III-V family, make good candidates for the photocathodes, as they have well defined energy band structures and they can obtain high photoemission yields [1.2]. The yield of the photocathode is otherwise known as the quantum efficiency (QE), and is defined as the number of electrons emitted per incident photon. The QE of any photocathode is dependent on the wavelength of incident light as well as on the surface state of the material. GaAs is treated to have a negative-electron-affinity (NEA) level at its surface while in use as a photocathode. In order to create polarized electron beam, the photocathode is illuminated with circularly polarized light of photon energy equal to the semiconductor band gap. There is an inherent trade off in increasing the figure of merit, $\mathrm{IP}^{2}$, of an accelerator by changing the wavelength of illumination. Shorter wavelengths (relative to the band gap) increase the QE of a cathode while simultaneously decreasing the polarization of the electron beam.

Polarized electron beams are created via photoemission of electrons from particular spin states in GaAs. By illuminating the photocathode with circularly polarized light, only electromagnetic transitions between the conduction band and valence band of GaAs which conserve angular momentum are allowed, in which $\Delta \mathrm{m}=+/-1$. For unstrained, bulk GaAs crystal, the highest theoretical polarization is $50 \%$, with values of $35 \%$ common experimentally. The polarization is limited by a degeneracy in the heavy hole $(\mathrm{HH})$ and light hole $(\mathrm{LH})$ states at the $\Gamma$ point (momentum vector $\mathbf{k}=0$ ) of the GaAs valence band (Figure 2.3) $[1.3][1.4]$. In order to increase the electron beam polarization, past efforts have focused on eliminating this degeneracy by breaking the crystal symmetry. A $50 \mathrm{meV}$ splitting of the bands can be obtained by growing a thin layer of GaAs on $\mathrm{GaAs}_{1-\mathrm{x}} \mathrm{P}_{\mathrm{x}}$, which introduces a compressive biaxial strain, and a typical polarization of $75 \%$. Current state of the art in GaAs cathodes involves so called "superlattice" GaAs, which is created of many alternating thin layers of GaAs and GaAsP, and which provides $85 \%$ polarization [1.5][1.6][1.7][1.8]. 
CHAPTER 1: INTRODUCTION

High polarization photocathodes are very expensive compared to bulk GaAs. A less expensive way to create highly polarized beams could bring interesting physics into small and university labs, and as such, an alternative way of creating electron beams from superlattice GaAs is desired. Using very simple quantum mechanical selection rules, Matsuyama proposed that the absorption of two photons, each with half the band gap energy and circularly polarized with the same helicity, would only allow the promotion of one electron within the degenerate bands of bulk GaAs into the conduction band Figure 4.1 [1.9]. This dissertation is the first published experiment to measure the polarization of a photoemitted electron beam produced via this two photon process, which was measured using a new style of retarding field microMott polarimeter commissioned in anticipation of this experiment.

Initially, two-photon absorption did not provide high polarization; rather polarization was lower than produced via one photon absorption. The electron beam polarization was measured for several different thickness of bulk GaAs using both one and two photon absorption processes. For one photon absorption, polarization was always less than $50 \%$ and the polarization increased while using thinner material, as expected [1.10]. For two photon absorption, polarization from bulk GaAs was approximately half that due to one photon polarization. Polarization improved using thinner photocathode material, with the polarization due to two photon absorption approaching that due to one photon, although it was always less than that obtained via one photon absorption. It is reasonable to attribute reduced polarization to production of electron beam from deeper within the material, as the farther the electrons travel, the more they are known to depolarize. This result builds on past experimental work designed to better understand depolarization mechanisms [1.10].

As important as GaAs is to the nuclear physics accelerator community, it does have several disadvantages. For accelerators that do not require unpolarized electrons, the problems of GaAs, namely it's highly stringent vacuum and handling requirements, low charge lifetimes, and long electron pulse lengths, necessitate the investigation of other photocathode materials. Of recent interest are the alkaliantimonide photocathodes, and in particular, $\mathrm{K}_{2} \mathrm{CsSb}$. Because these cathodes are not treated to create a 
NEA surface, it is thought that they will be more robust under poor vacuum conditions and in the presence of ion damage than GaAs. A robust QE under modest vacuum requirements would make $\mathrm{K}_{2} \mathrm{CsSb}$ an ideal source for high current accelerator applications that do not require polarized electrons, such as light sources, energy recovery linacs, and electron cooling machines.

Together with Brookhaven National Laboratory (BNL), a thin film $\mathrm{K}_{2} \mathrm{CsSb}$ photocathode was manufactured and installed into the test photogun at JLab. To confirm the resistance to ion damage and vacuum conditions, charge lifetime measurements of $\mathrm{K}_{2} \mathrm{CsSb}$ were compared to GaAs under identical conditions. Preliminary results showed that the charge lifetimes were approximately the same as with GaAs. Further investigation indicated improved lifetimes, which were dependent on the amount of laser intensity incident on the photocathode. Additional studies indicated that the heating of the photocathode surface caused by the incident laser could either positively or negatively impact the charge lifetime of $\mathrm{K}_{2} \mathrm{CsSb}$, depending on the intensity.

The primary purpose of this dissertation is to investigate properties of semiconductors that are used as photocathodes in electron accelerators. By understanding phenomenon that result in depolarization from maximum theoretical polarization and the loss of QE from a photocathode, it is hoped that new techniques and materials can be developed that will yield higher polarization and higher beam intensity. The dissertation will describe a load locked low energy electron source that was used with the calibration and commissioning of the attached new style compact micro-Mott polarimeter. This polarimeter was used to study the polarization of electron beams generated from bulk GaAs via both one and two photon absorption as it related to the maximum depth of electron excitation. A theoretical model of the depolarization effects of the electron transport to the surface will also be detailed. Also described will be the test stand, both the photogun and beamline, used for studying $\mathrm{K}_{2} \mathrm{CsSb}$ as well as how its charge lifetime compares to that of GaAs. The charge lifetime of $\mathrm{K}_{2} \mathrm{CsSb}$ was observed to be highly dependent on the amount of laser heating of the substrate. Studies of surface morphology, as well as a 
CHAPTER 1: INTRODUCTION

thermal model, will be presented that further explain the effects of charge lifetime varying with the amount of laser heating. 


\section{Photocathode Theory}

Electron beams have important roles in fields ranging from basic science research to defense. These beams are common not only in scientific endeavors, electron microscopy, and medical devices, they are also used in consumer goods such as microwave ovens and cathode ray tube televisions; electron beams are truly used universally. Of interest to Jefferson Lab, and in particular the CIS, is the generation of electron beams for the study of nuclear physics. There are currently two major ways of generating the electron beams used in accelerators, and while thermionic emission is currently the most common for generating electrons, JLab uses photoemission.

Photoemission has many key advantages over thermionic emission, as related to the nuclear physics needs of JLab. Electron beams created via photoemission can consist of short, picosecond length pulse bunches of electrons, which can be emitted at high repetition rates and high duty factors, which is a beam structure unobtainable with thermionic sources and needed for nuclear physics. JLab currently uses a semiconductor material for its photocathode, GaAs, which is the current photocathode of choice for polarized electron beams. This chapter will discuss the theoretical underpinnings of semiconductor photocathodes, as well as commonly used figures of merit and operational parameters for photocathodes. Section 2.1 will quickly cover the basics of photocathodes, while Section 2.2 discusses the production and emission of electrons in GaAs. 


\subsection{Photocathode Basics}

\subsubsection{Quantum Efficiency}

To begin a discussion of photocathodes, a few figures of merit must first be discussed, in order to give the different types of photocathodes additional context. The first, and possibly the most important, figure of merit is known as the quantum efficiency $(\mathrm{QE})$, which, at its simplest, is simply the fraction of photons incident on the photocathode that result in a photoemitted electron. This definition assumes that at a maximum only one electron can be excited per photon, which is valid assumption for low energy photons, such as those near the band-gap of semiconductor. QE is a useful metric as it includes many characteristics of photocathode in one number: optical characteristics, such as reflectance and absorption: electron transport characteristics, such as scattering effects and mean free path: and surface energy barrier characteristics, such as the work function for metals and electron affinity for semiconductors [2.1]. As $\mathrm{QE}$ is essentially a ratio of photons to electrons, it is taken to be unitless. To calculate the QE, several measurements must be made, and then related using physical constants, using the formula

$$
\mathrm{QE}=\frac{\mathrm{hcI}_{\lambda}}{\mathrm{q} \lambda \mathrm{P}_{\lambda}}
$$

which uses Planck's constant, the speed of light, and the electron charge combined with the measured photocurrent, $\mathrm{I}_{\lambda}$, optical power, $\mathrm{P}_{\lambda}$, and wavelength of light used, $\lambda$. For one-photon effects, $\mathrm{QE}$ will generally be constant with regards to light intensity, as more light will produce more photocurrent, with the ratio defined by the $\mathrm{QE}$. The relationship between optical power and photocurrent will change at very high levels of either, as nonlinear effects are introduced. Because photocathodes are used to generate electron beams, a high QE is desired, and in practice QE's range from $0.01 \%$ in metals in the UV, to $8 \%$

for $\mathrm{K}_{2} \mathrm{CsSb}$ in the green, to $15 \%$ and higher for GaAs in the green. For the case of polarized beams, QE directly impacts the figure of merit, $\mathrm{IP}^{2}$, as a high $\mathrm{QE}$ allows for more current with any given laser power. 
CHAPTER 2: PHOTOCATHODE THEORY

\subsubsection{Lifetime}

While a high QE generally makes for a better photocathode, as it can deliver more beam with less lower power, it is not the only important metric for photocathodes. Another relevant feature of any photocathode used to supply beam to an accelerator is the amount of beam it can deliver before it no longer works. A photocathode with very high $\mathrm{QE}$ is of little use if the $\mathrm{QE}$ decays to $0 \%$ before any useful science can be done. A metric often used to describe the length of service of a photocathode is known as the lifetime, and there are two lifetimes often quoted. The first lifetime is known as dark lifetime and refers to the length of time a photocathode can sit with no exposure to light in a given vacuum environment before its QE falls to a value of 1/e of its original value. The dark lifetime gives a good indication as to the sensitivity of the surface of the cathode to chemical reactions due to residual gases in the vacuum chamber. Also of importance is operational lifetime, and for the purposes of this dissertation, operational lifetime will be defined as charge lifetime, which is the amount of charge that can be extracted from the photocathode before its QE falls to a value of 1/e of its original value. Referencing the operational lifetime to extracted charge instead of to actual time generating beam allows for cross comparison between electron sources that deliver very different amounts of current at different duty factors and repetition rates.

The charge lifetime of modern GaAs photoguns is thought to be limited by the process of ion bombardment [2.2]. In ion bombardment, residual gases in the photogun are ionized by the extracted electron beam, and these ions are then accelerated backward towards the photocathode. The actual mechanism by which these incident ions degrade the QE of a NEA GaAs photocathode is unknown, but it is known that they can penetrate the surface of the cathode [2.3]. The ions could damage the crystalline structure and reduce the electron diffusion length, which according to Spicer's model (Section 2.3) reduces the QE, or they could change the photocathode energy structure. The ions could also serve to 
CHAPTER 2: PHOTOCATHODE THEORY

sputter away the chemicals that form the NEA surface, which would increase the electron affinity of the surface.

No matter the mechanism of QE loss, the experience of the CIS group and other electron gun groups is that the charge lifetime can be increased by improving the vacuum in the photogun, which then reduces the amount and thus the probability that any given gas species will become an impinging ion on the photocathode surface. Typical GaAs photogun are run in the mid $10^{-12}$ Torr pressure regime, which requires time, money, and experience to achieve. Also of note is the lack of successful use of GaAs in warm RF photoguns due to its extremely strict vacuum requirements.

Lifetimes, and some of the factors that influence them, will be discussed more fully in Chapter 5. In brief, a longer lifetime is desirable, as it extends the useful operational time before a photocathode must be replaced or refreshed, which are usually time consuming processes that lead to downtime of scientific programs.

\subsection{GaAs Properties}

GaAs is of the III-V family of direct band-gap semiconductors. These materials make for ideal photocathodes, as they have a high photon absorption coefficient, long electron diffusion length, and, with minimal surface preparation, they can create a negative electron affinity (NEA) surface state, which enhances their photoemission yield [1.2]. In NEA materials, electrons excited to the surface state escape readily into the vacuum, as the vacuum level is below that of the conduction band of the material. GaAs has the highest direct band-gap of the III-V semiconductors, which makes it highly suited to the production of electron beams. A high band-gap reduces the dark current of a photogun [2.2], and it also creates a larger NEA level, as the conduction band is further separated from the treated surface, which

enhances photoemission further [2.5]. This section discusses the relevant properties of GaAs semiconductors as they relate to crystal structure, energy bands, surface states, and polarization. 


\subsubsection{Crystal Properties}

The crystal lattice of GaAs has zincblende symmetry, in which every atom lies at the center of a tetrahedron, with four other atoms at each corner [2.7]. Two electrons with opposite spin bind thee two nearest neighbors in the crystal. This zincblende lattice is considered an interpenetrating face centered cube, also known as a face centered cube with atoms inside. Figure 2.1 shows a conventional unit cube for GaAs and Figure 2.2 [2.8] shows the lattice structure of the three most basic symmetry planes, with the (100) plane used in the photocathodes of the CIS. It should be noted that at very high temperature, the (100) plane can reconstruct to the (110) plane [2.9]. Because of this reconstruction, care must be taken during the preparation of GaAs for use as a photocathode as process involves surface cleaning via heat.

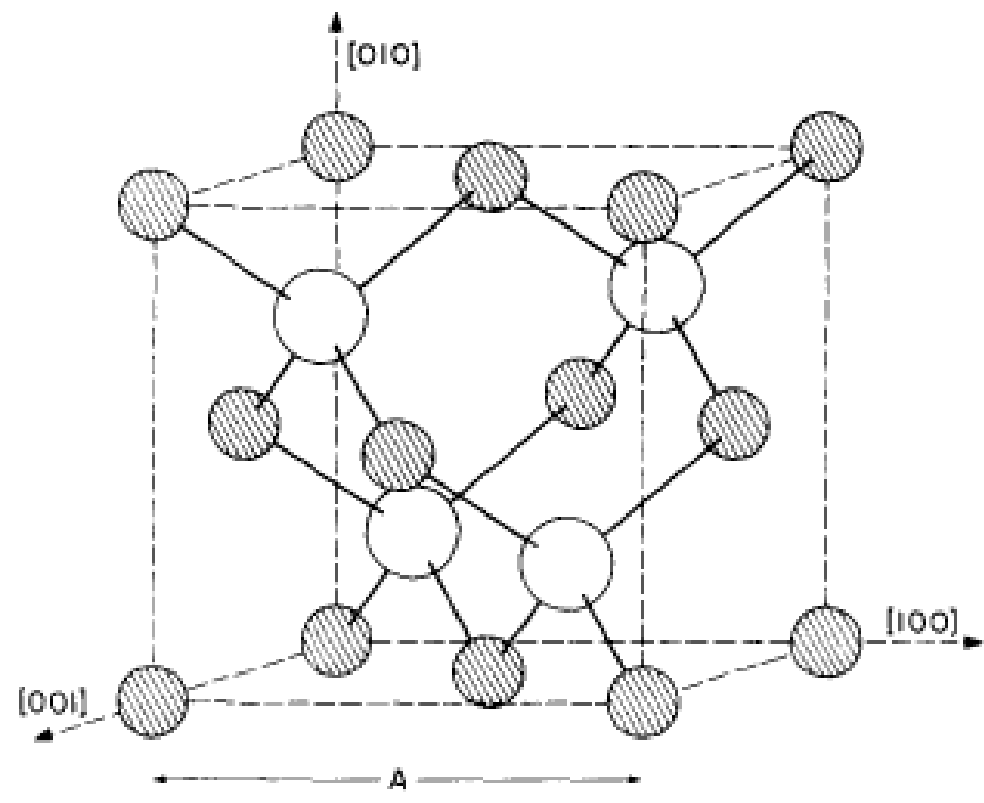

Figure 2.1: Conventional unit cube for GaAs, from Ref. [2.5] 

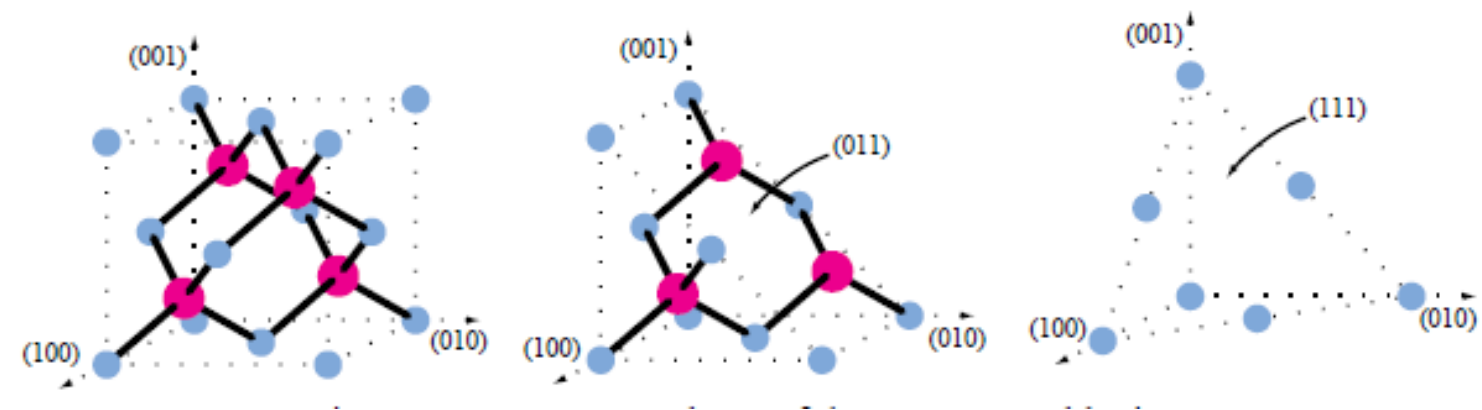

Figure 2.2: Symmetry planes of the GaAs crystal, from Ref. [2.8].

\subsubsection{Energy Bands}

By using a periodic potential that represents the lattice structure, the Schrödinger equation can be solved to give the energy-momentum relationship of an electron in a periodic crystal. For GaAs, along with most semiconductors, the solution to this equation gives two sets of energy bands, known as the conduction and valence bands, which represent upper and lower energy levels, respectively. These bands are separated by a region known as the band-gap, in which no states are allowed to exist. The band structure of GaAs is shown in Figure 2.3. The upper valance bands have angular momentum symmetry $\mathrm{L}=1$, and with spin included the bands split due to the spin-orbit interaction breaking the symmetry between the $\mathrm{p}_{3 / 2}$ and $\mathrm{p}_{1 / 2}$ bands. At the $\Gamma$ point, the top two valance bands, known as the heavy-hole and light-hole bands, are degenerate. Also located at the $\Gamma$ point is the minimum of the conduction band, which has only spin degeneracy. Because the bottom of the conduction band matches the top of valence band at $\mathbf{k}=0, \mathrm{GaAs}$ falls in the category of direct transition semiconductors, with a band-gap of around $1.42 \mathrm{eV}$ at the $\Gamma$ point. [2.5][2.7][2.8]. 


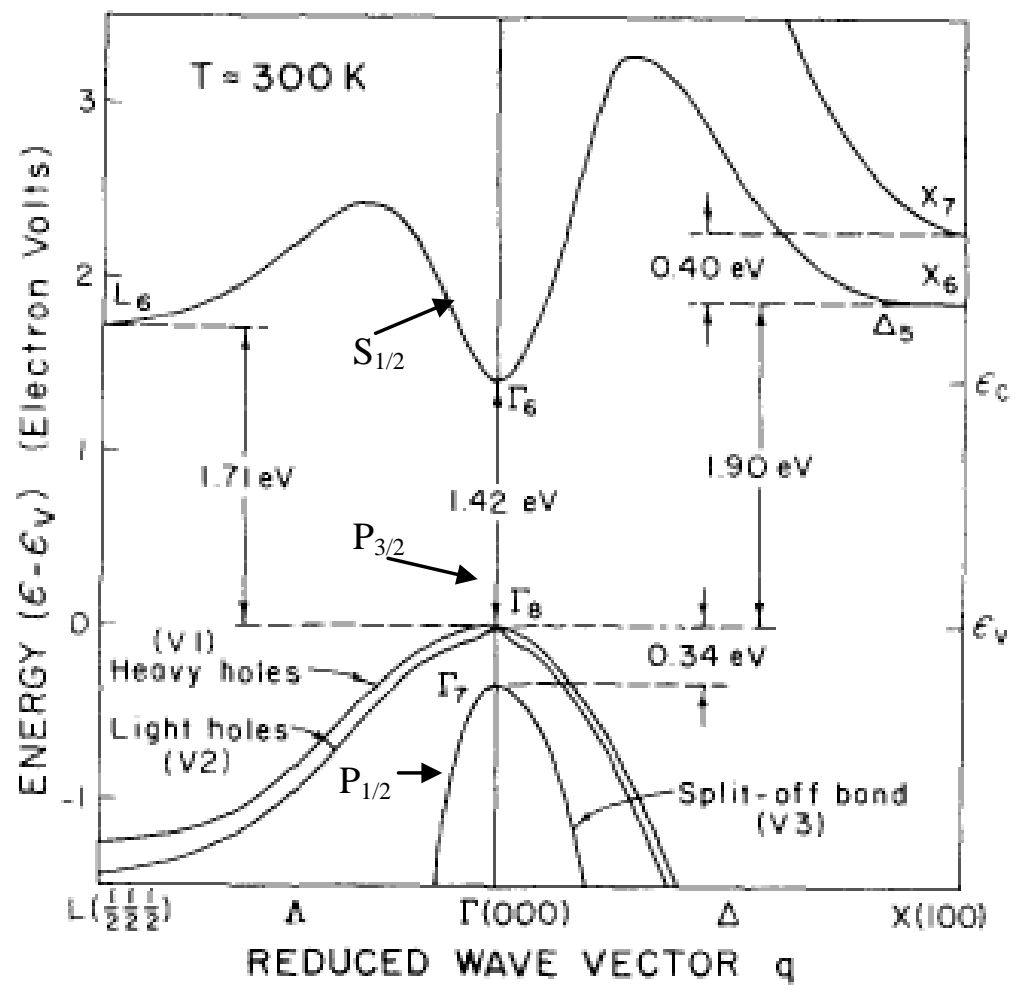

Figure 2.3: Energy band diagram for intrinsic GaAs. Only shown are the uppermost part of the valence band system and the lowest sets of conduction band minima. Energy gaps are shown as appropriate for room temperature, from Ref. [2.10].

\subsection{Photoemission Model}

\subsubsection{One Photon Photoemission}

A simple Three-Step Model of photoemission in semiconductors was first successfully explained by William Spicer [1.2]. As the name implies, this model proposes that photoemission consists of three main steps: the photoexcitation of electrons into the conduction band, the transport of these electrons to the surface, and the emission of electrons into the vacuum. The three steps of photoemission are shown in Figure 2.4. Only electrons with energies greater than the vacuum level can escape the surface, and the 
energy distribution of the photoexcited electrons produced inside of the material will decrease as electrons reach the surface.

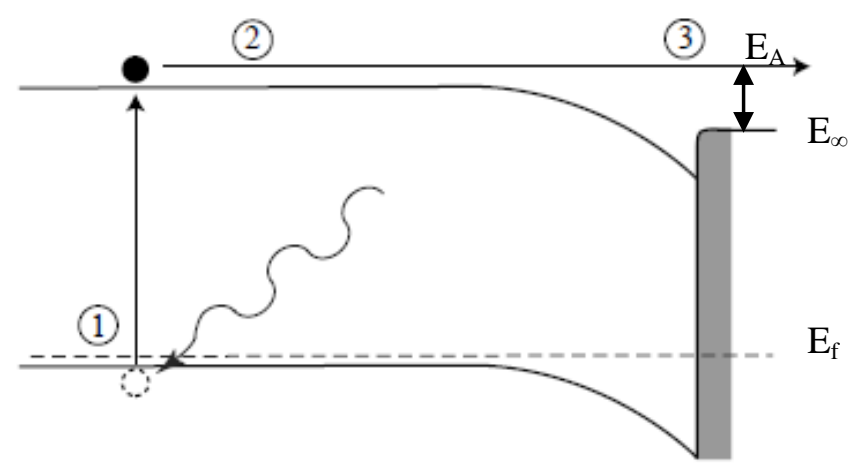

Figure 2.4: Spicer's Three-Step Photoemission Process: 1 - photoexcitation of valence electrons into the conduction band (creation of electron -hole pair), 2 -transport of electrons to the surface, 3 - emission of electrons into the vacuum.

The first step of Spicer's model requires the absorption of light by the electron. The higher the optical absorption coefficient, the more likely any given photon will excite an electron, and as such good electron emitters require high coefficients. Direct band gap semiconductors, such as GaAs, have high absorption coefficients, and as shown by Figure 2.5, the threshold of photoemission is marked by a knee shape at the band gap energy, which for GaAs occurs near $1.42 \mathrm{eV}$, or $872 \mathrm{~nm}$. 


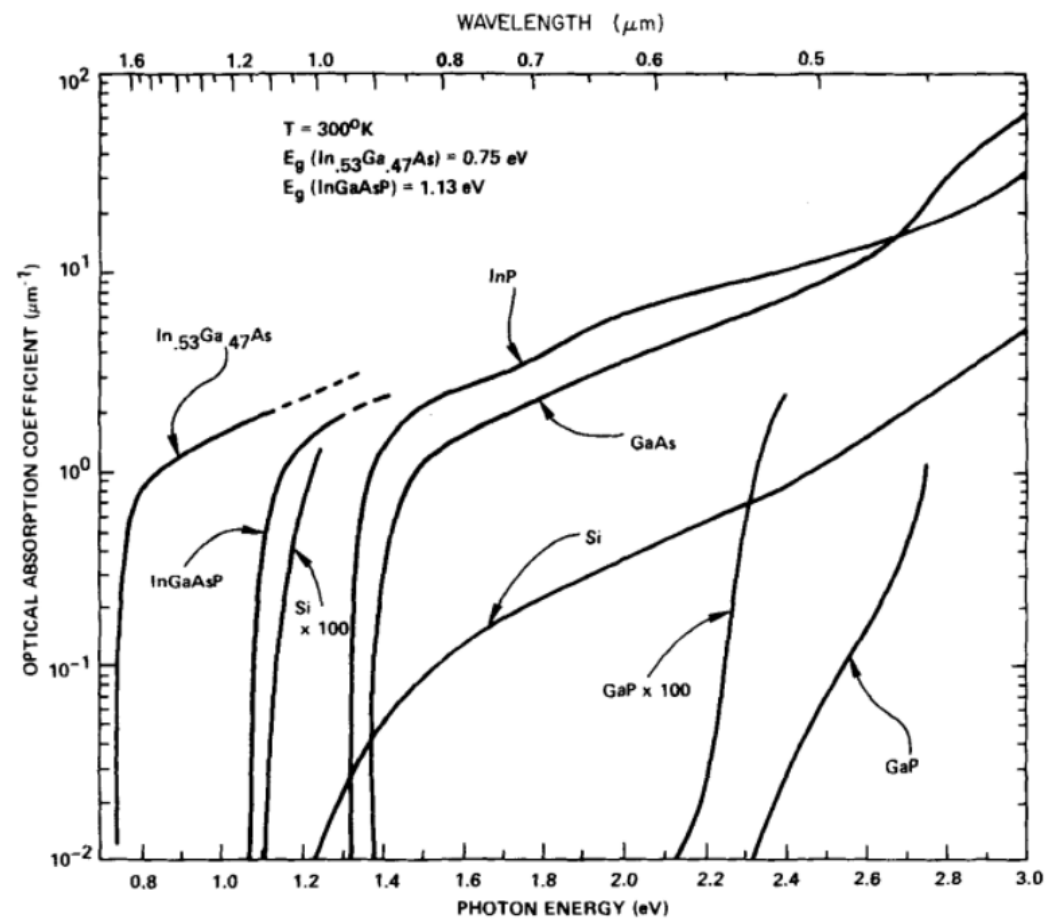

Figure 2.5: Optical absorption coefficients for some NEA semiconductors from Ref. [2.11]. The pronounced knees mark the photoemission threshold at the band-gap energy. This band-gap energy is at $1.42 \mathrm{eV}$ for GaAs.

The transport of photoexcited electrons to the surface is the process that significantly differentiates the photoemission of metals and semiconductors. In metals, the conduction band minimum is below the Fermi level, which creates many occupied conduction band states. Photoexcited electrons will scatter more quickly, thus losing too much energy to be emitted into vacuum. However, the conduction band of semiconductors is mostly empty, allowing for minimal electron scatter; the band gaps of some semiconductors are even high enough to suppress almost all thermionic excitation to the conduction band.

Spicer's model also indicates that a good photoemitter will have a low electron affinity, defined as the potential difference between the vacuum level and the bottom of the conduction band, as this low affinity will increase the probability of electron emission. Good photocathodes have high work functions, defined as the potential difference between the vacuum level and the Fermi level. A high work function creates a potential barrier that hinders the emission of electrons excited by purely thermal energy. 
CHAPTER 2: PHOTOCATHODE THEORY

As mentioned previously, the effectiveness of a photocathode is often quantified by measuring its QE, which is the ratio of emitted electrons to incident photons. Assuming a uniform half-infinite semiconductor, Spicer's theory can predict the QE of a semiconductor, and simplifies to:

$$
Q E=(1-R) \frac{\frac{\alpha_{P E}}{\alpha} P_{E}}{1+\frac{l_{a}}{L}}
$$

Here $\mathrm{R}$ is the reflectivity of the surface of the material, $\alpha_{\mathrm{PE}}$ is the absorption coefficient for electrons excited above the vacuum level, $\alpha$ is the absorption coefficient for the semiconductor, $\mathrm{P}_{\mathrm{E}}$ is the escape probability of electrons reaching the surface, $l_{\mathrm{a}}$ is the photon absorption length $(=1 / \alpha)$, and $\mathrm{L}$ is the diffusion length. The fraction $\alpha_{\mathrm{PE}} / \alpha$ just gives the ratio of electrons excited above the vacuum level, while $l_{d} / \mathrm{L}$ gives the ratio of absorption length to diffusion length. These variables are all dependent on incident light wavelength, temperature, and doping concentration. Of particular note is that the QE is inversely proportional to $1+l_{\mathrm{a}} / \mathrm{L}$, which indicates that electrons excited deep inside of a material will likely not be photoemitted.

\subsubsection{Two Photon Photoemission}

While Spicer's model is primarily for one photon absorption processes, it can be modified to accommodate a non-linear two photon process. Two photon absorption can only occur in crystals that lack inversion symmetry, such as GaAs (the potential changes when As and Ga are exchanged in the crystal lattice) [2.12]. The cross-section for this process is small because there is only a small likelihood that two photons with energy less than the band gap can simultaneously excite the same electron from the valence to conduction band. Changing the absorption coefficients of Spicer's model to clearly indicate 
CHAPTER 2: PHOTOCATHODE THEORY

that the absorption requires the simultaneous absorption of two photons in both space and time indicates that the QE will be dependent on the intensity of the incident light. The linear QE dependence of the two photon process on laser intensity is then unlike the QE dependence of the one photon process on intensity, which is a constant. After an electron is excited to the conduction band via two-photon absorption, the rest of the photoemission process will be identical to that of one-photon absorption; the electron transport and surface escape mechanisms will not differentiate between electrons excited via one- or two- photon absorption. Two-photon absorption will be discussed in greater detail in Chapter 4.

\subsection{NEA and PEA Photocathodes}

The escape probability of an electron from a photocathode into vacuum can be greatly enhanced by creating a NEA surface on a photocathode. NEA will occur when the vacuum level lies below the conduction band minimum. Because GaAs, and III-V type semiconductors in general, has the capability to create the NEA state, it is an extremely efficient photocathodes. The surface is prepared by creating a surface dipole by adding monolayer quantities of an alkali metal, such as Cs, which donates its electron to the semiconductor and leaves a positive charge on the surface, and lowers the affinity to nearly zero [2.13]. The vacuum level can be lowered even further by adding an electronegative element, such as F, to the surface. For this technique to work, a heavily p-typed doped crystal is needed, as the doping pins the Fermi level near the valence band maximum and the bands then bend downward in energy near the surface. To produce this NEA effect, a UHV environment is required to avoid sub monolayer contamination of the surface. Figure 2.6 shows the formation of the NEA surface using GaAs and monolayers of $\mathrm{Cs}$ and $\mathrm{F}$. 


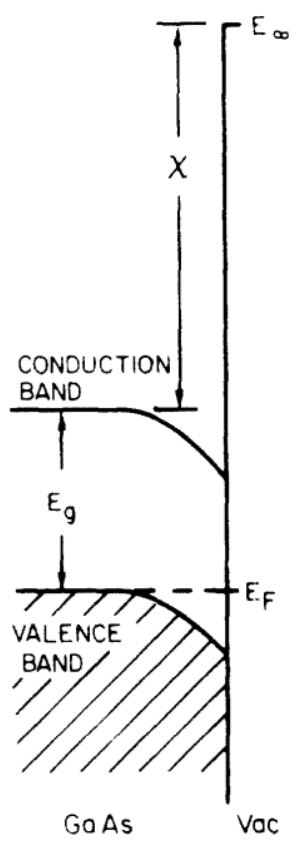

(a)

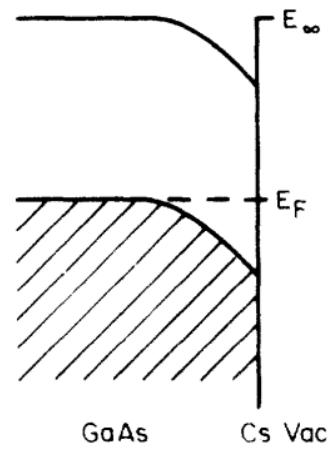

(b)

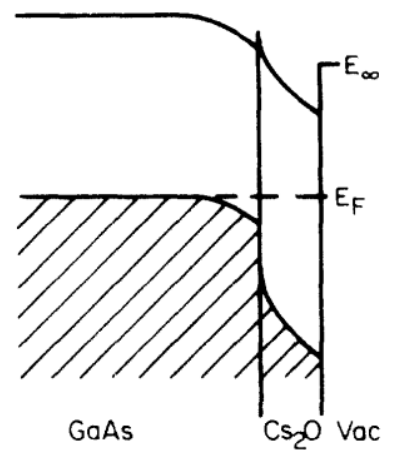

(c)

Figure 2.6: Formation of a NEA surface. p-doping GaAs lower the semiconductor vacuum level via band bending at the surface. Addition of a $\mathrm{Cs}$ and $\mathrm{O}$ (or other oxidant) monolayer lower the vacuum level below the conduction band minimum, from Ref. [2.13].

In contrast to NEA surfaces are positive electron affinity surfaces (PEA), which as their name implies, have a positive electron affinity. While electrons in NEA cathodes are accelerated as they pass through the Cs-F surface, electrons in PEA surfaces at the conduction band minimum cannot escape into vacuum. Because electrons must have energy higher than the conduction band minimum, the threshold photon energy is raised for a PEA cathode. In addition, to escape the PEA surface, the electrons must come from an escape cone, in order to ensure that they have sufficient momentum normal to the surface [2.13]. These effects are shown in Figure 2.7. While some natural PEA photocathodes, such as GaAs, can be turned into NEA photocathodes via the addition of surface dipoles, it is important to note that not all photocathodes with PEA can be turned into NEA photocathodes. 


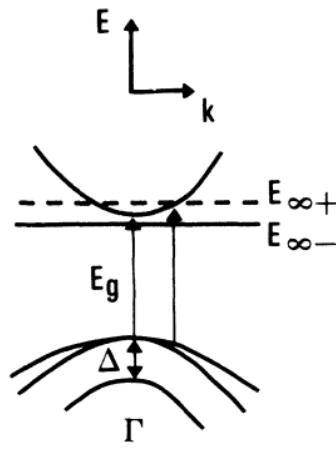

(a)
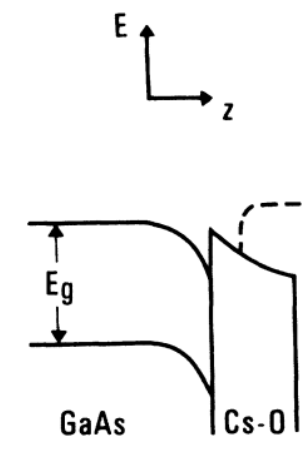

(b)

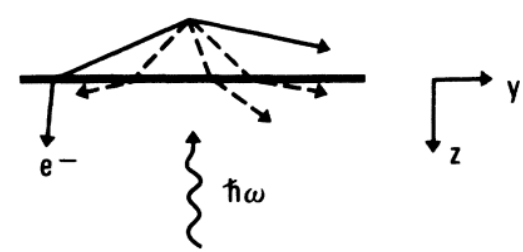

(c)

Figure 2.7: Photoemission from PEA and NEA GaAs compared in three different spaces: (a) $E$ - vs $k$, (b) $E$ vs $z$, and (c) $z$ vs $y$. The dashed line corresponding to the PEA case represents the vacuum level Eœ+ in (a), and the surface barrier in (b). The higher levels in the PEA case result in the electron emission being confined to an escape cone in the material (c) and the electrons losing energy at the surface. In contrast, in the NEA case the electron emission is not confined to a cone in the material and the electrons gain energy during emission. From Ref. [2.13].

\subsection{Polarized Electrons}

The orderly band structure of GaAs allows the promotion of electrons in a specific spin state to the conduction band, and thus allows the creation of polarized electron beam. Illuminating GaAs with circularly polarized light at the band gap energy, as shown in Figure 2.8 leads to an electron beam polarization of $50 \%$. The relative transition probabilities of 3 to 1 for the ${ }^{2} \mathrm{P}_{3 / 2}$ states of $\mathrm{m}_{\mathrm{j}}=3 / 2$ and $1 / 2$ respectively arise from computing the matrix elements of transitions probabilities between the states as caused by the circular light. The 3 to 1 ratio is what yields the inherent maximum polarization of

$$
P=\frac{3-1}{3+1}=50 \%
$$


The polarization of bulk GaAs is limited to $50 \%$ because of the heavy hole - light hole degeneracy in the ${ }^{2} \mathrm{P}_{3 / 2}$ at $\mathbf{k}=0$. Because the figure of merit for polarized sources goes as $\mathrm{IP}^{2}$, much work has gone into increasing the $50 \%$ polarization from bulk GaAs. Current techniques of increasing the polarization from GaAs, which involve introducing a strain in the GaAs crystal lattice, as well as a newly proposed method using two-photon absorption, will be discussed more thoroughly in Chapter 4.

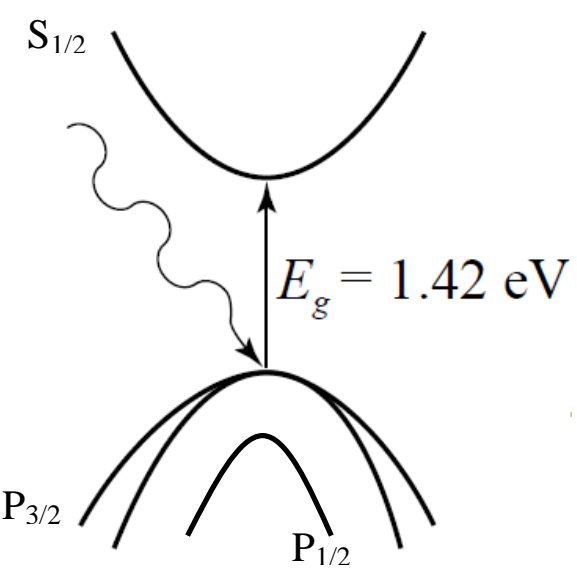

Conduction band

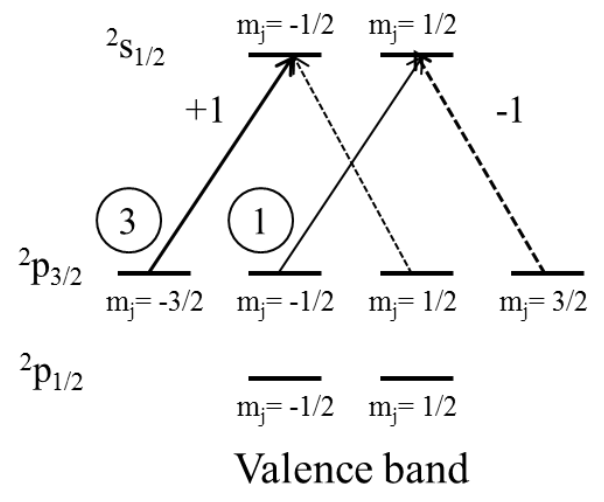

Figure 2.8: Optical transitions in GaAs with circularly polarized light from the ${ }^{2} \mathrm{P}_{3 / 2}$ valence band to the ${ }^{2} \mathrm{~S}_{1 / 2}$ conduction band. The circled values indicate transition probabilities. Maximum polarization from un-strained bulk GaAs would be $50 \%$ for one photon excitation due to degeneracy between the heavy hole - light hole states.

\subsection{Depolarization Mechanisms}

For semiconductors with medium to high doping levels, such as the GaAs used in the following experiments, there are two major depolarization mechanisms which occur in the bulk material at near room temperatures. These two mechanisms are the exchange interaction between electrons and holes, which known is the BAP process (after G.L. Bir, A.G.Aronov, and G.E. Picus), and the narrowing of the magnetic resonance in spin-orbit split-off conduction bands, which is known as the DP process (after M.I. Dyakonov and V.I.Perel). 
CHAPTER 2: PHOTOCATHODE THEORY

For room temperatures, the DP process is the dominant depolarization mechanism for doping levels much less the levels of the GaAs used in the following work. The DP process mechanism arises in crystals which lack inversion symmetry (such as GaAs). This asymmetry breaks the conduction band degeneracy for $\mathbf{k} \neq 0$ for any direction besides (100) and (111). This splitting of the conduction band is akin to an internal magnetic field, which is then dependent on $\mathbf{k}$. Typical values for spin relaxation rates for this process are between $4 \times 10^{9}$ and $2 \times 10^{10} \mathrm{~s}^{-1}$ if the doping concentration is less than $10^{17} \mathrm{~cm}^{-3}$ $[2.14][2.15]$.

The BAP process is the dominant bulk depolarization mechanism for the doping levels used in this work, which were concentrations greater than $10^{18} \mathrm{~cm}^{-3}$. Because the BAP process depends on the exchange interaction between electrons and holes, it is dependent on hole concentration, which is related to doping level, the velocity of the electron as it moves through the material, as well as the probability that each interaction between an electron and a hole will cause a spin flip. At high doping levels, typical spin relaxation rates for the BAP process are between $2-4 \times 10^{10} \mathrm{~s}^{-1}[2.14][2.15]$.

Also of note is the potential for the surface of the GaAs to cause electron depolarization. The symmetry of the bulk crystal is broken at the surface both by the surface itself and by the Cs-F layer, which can destabilize the spin states. While this mechanism is less understood than the BAP and DP processes, it is believed that during the photoemission process the ionized Cs atoms that create the NEA state act as spin scatterers [2.16]. 


\section{Mott Polarimeter}

Nuclear physics experiments have come to rely on high polarization electron beams, and place stringent demands on the electron source. In particular, beam requirements at the Thomas Jefferson National Accelerator Facility's CEBAF electron accelerator include polarization over $80 \%$ and average current capability of at least $100 \mu \mathrm{A}$. Access to the CEBAF polarimeters for photocathode research is heavily constrained by the experimental schedule which means that the majority of development must be done outside of the CEBAF tunnel. Previously, offline photocathode polarization research at JLab was performed using a $100 \mathrm{kV}$ electron gun and a conventional Mott polarimeter, which required extensive radiation shielding and a personal safety system. Described in this chapter is a simple, load-locked, lowvoltage polarized electron source used in conjunction with a newly designed compact, retarding-field Mott polarimeter. This polarimeter enables photocathode pre-qualification for the CEBAF injector, and for this dissertation it was used to study the two-photon absorption of GaAs. While the previous chapter discussed the theoretical electron beam polarization from GaAs, this chapter will discuss the measurement of the polarization using this new style of micro-Mott polarimeter. The design and commissioning of the polarimeter will be discussed, along with the physics underpinning its mechanics.

\subsection{Theory of Mott Polarization Measurement}

Many polarization measurements rely on detecting an asymmetry between states. N.F. Mott in the 1920s considered the scattering of relativistic electrons from nuclei [3.1]. As the incident electron scatters with a velocity $\mathbf{v}$ near a high- $Z$ atom, the electron field $\mathbf{E}$ of the nucleus causes a magnetic field $\mathbf{B}$ in the electron rest frame, which is given by; 


$$
\mathbf{B}=-\frac{1}{c} \mathrm{v} \times \mathbf{E}
$$

Letting the nucleus to electron distance be $\mathbf{r}$, taking the Coulomb field of the nucleus to be $\mathbf{E}=\left(\mathrm{Ze} / \mathrm{r}^{3}\right) \mathbf{r}$ and taking the standard orbital angular momentum to be $\mathbf{L}=m \mathbf{r} \times \mathbf{v}$, Eq. 3.1 can be rewritten as;

$$
\mathbf{B}=\frac{\mathrm{Ze}}{c r^{3}} \mathbf{r} \times \mathbf{v}=\frac{\mathrm{Ze}}{m c r^{3}} \mathbf{L}
$$

This magnetic field interacts with the electron spin magnetic moment $\mu$, and introduces a term $V_{s o}=-\mu$. B in the scattering potential, where $\mathrm{V}_{\text {so }}$ can be written using the electron spin $\mathrm{S}$ as;

$$
V_{\text {so }}=\frac{\mathrm{Ze}^{2}}{2 m^{2} c^{2} r^{3}} \mathbf{L} \cdot \mathbf{S}
$$

This $\mathrm{V}_{\text {so }}$ term represents spin-orbit coupling, which introduces an asymmetry dependence on electron spin in the cross section for electrons polarized transverse to the scattering plane. The differential cross section is;

$$
\sigma(\theta, \varphi)=I(\theta)(1+S(\theta) \mathbf{P} \cdot \hat{n})
$$

where $\mathbf{S}(\theta)$ is the asymmetry cross section, $I(\theta)$ is the spin-averaged scattering intensity, and $\mathbf{P}$ is the incident electron polarization. $\hat{n}$ is the unit vector normal to the scattering plane, as defined by the incident and scattered electrons. 
CHAPTER 3: MOTT POLARIMETER

The asymmetry cross section, which is also known as the Sherman function, is best understood through the use of two examples. An unpolarized electron beam can be understood to have equal numbers of electrons with spin parallel (spin-up) and with spin antiparallel (spin-down) as compared to $\hat{n}$. After scattering through an angle $\theta$, it follows from Eq 3.4 that the number $\mathrm{N}_{\uparrow}$ of spin-up electrons is proportional to $1+S(\theta)$, while the number $N_{\downarrow}$ of spin-down electrons is proportional to $1-S(\theta)$. This difference in scattering intensity leads to a net scattered electron polarization $P(\theta)$ given by;

$$
P(\theta)=\frac{\mathrm{N}_{\uparrow}-\mathrm{N}_{\downarrow}}{\mathrm{N}_{\uparrow}+\mathrm{N}_{\downarrow}}=\frac{(1+\mathrm{S})-(1-\mathrm{S})}{(1+\mathrm{S})+(1-\mathrm{S})}=\mathrm{S}(\theta)
$$

The asymmetry in the cross section caused the originally unpolarized beam to become polarized, with a value equal to the Sherman function.

A beam of electrons with a polarization $P$ transverse to the scattering plane will form the second example. From Eq 3.4, the number of electrons $\mathrm{N}_{\mathrm{L}}\left(\mathrm{N}_{\mathrm{R}}\right)$ scattered through an angle $\theta$ to the left(right), is proportional to $1+\mathrm{PS}$ and 1-PS, respectively. The measured scattering asymmetry, A, between two detectors located at equal and opposite scattering angles will be;

$$
A(\theta)=\frac{N_{L}-N_{R}}{N_{L}+N_{R}}=\operatorname{PS}(\theta)
$$

The Sherman function thusly relates the value of measured scattering asymmetry to the electron polarization. If the Sherman function is known, measuring the asymmetry gives the electron polarization component perpendicular to the scattering plane.

The Sherman function has only been calculated for single atom scattering, which means it cannot be used to directly measure real systems, even those of the thinnest foils. Because of multiple and plural 
CHAPTER 3: MOTT POLARIMETER

scattering effects, the effective Sherman function, $S_{\text {eff }}$, is smaller for real systems than for single atom scattering. The measured scattering asymmetry of a system is thus given by a slightly modified Eq. 3.6;

$$
A=\mathrm{PS}_{\mathrm{eff}}(\theta)
$$

Usually, $S_{\text {eff }}$ is determined for a particular foil target thickness and unknown polarization by extrapolating the measured asymmetry and multiple foil thicknesses to a hypothetical zero-thickness foil [3.2]. From this point, the true Sherman function can be used to determine the unknown polarization. Once the polarization is known, the effective Sherman function for each foil can be calculated. This double scattering technique is not ideal, and systematic errors tend to limit the accuracy of conventional foil Mott

polarimeter to $\sim 5 \%$ [3.3]. These large systematic errors are one reason that CIS found it necessary to commission a new form of micro-Mott polarimeter, the $S_{\text {eff }}$ of which was determined by using a scattered electron beam of known polarization.

\subsection{MicroMott Polarimeter Design and Techniques}

The vacuum apparatus consisted of three chambers (Figure 3.1a): a low voltage polarized electron source chamber, a beam transport section, and a micro-Mott retarding field style polarimeter. 


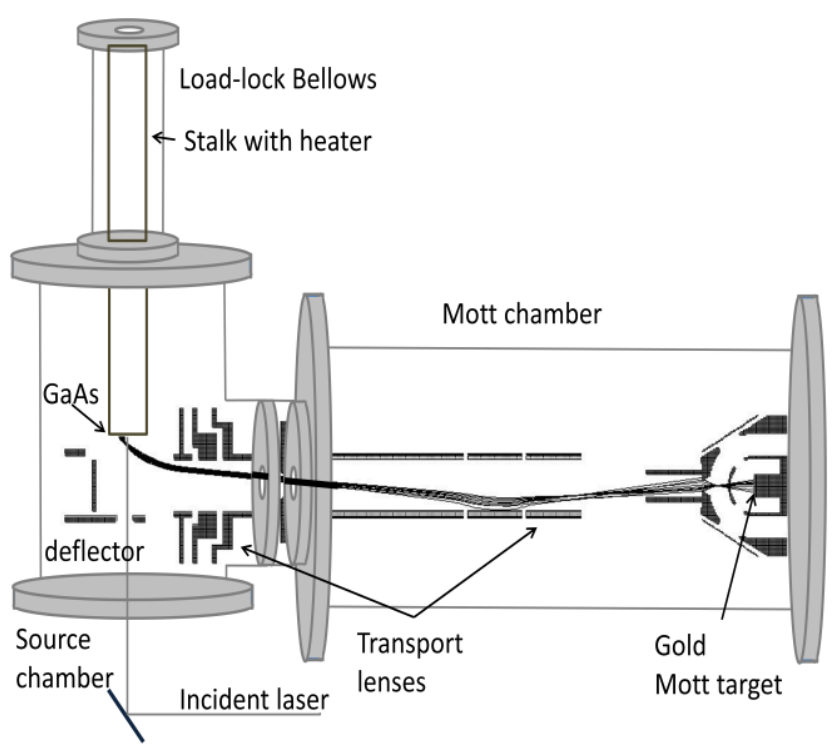

Figure 3.1: Schematic of the experimental apparatus.

\subsubsection{Apparatus and Design}

The polarized electron source chamber was an ultra-high vacuum system, in which GaAs photocathodes reside. Un-strained GaAs was mounted to a long hollow stalk that could be inserted into the source chamber. New photocathodes mounted on this stalk were introduced into the chamber using a load-lock system, in which the load-lock bellows were baked at $250^{\circ} \mathrm{C}$ for $\sim 12$ hours. To activate the GaAs to a NEA surface, a heater in the hollowed stalk heated the GaAs to a surface temperature of $\sim 550 \mathrm{C}$ in order to remove contaminants from the surface. $\mathrm{Cs}$ and $\mathrm{NF}_{3}$, which was used to supply the $\mathrm{F}$ as an oxidant, were added in alternating doses in the 'yo-yo' method until the QE gains between yo-yo peaks increased by less than 5\%. In this method, the Cs is first continuously applied using commercially available strips [3.4] until photocurrent is detected on a biased photocathode, and then the Cs remains on until the QE drops to half of its initial maximum value. At this point, $\mathrm{NF}_{3}$ is introduced, via a leak valve, until the $\mathrm{QE}$ reaches another maximum. The leak valve is closed, and Cs is reapplied until the QE falls to roughly half of its recent maximum value, at which point the process is repeated. The GaAs photocathode was lowered into an 8 " diameter stainless steel chambers, which was pumped with a combination of ion and 
CHAPTER 3: MOTT POLARIMETER

non-evaporable getter pumps [3.5]. GaAs requires very high vacuum, and to achieve a pressure in the low UHV regime, the polarimeter was initially baked in a hot air oven for 30 hours at $200^{\circ} \mathrm{C}$, while the source chamber was baked to nearly $250^{\circ} \mathrm{C}$. Because of some Teflon components in the polarimeter, it was not able to be baked to a full $250^{\circ} \mathrm{C}$, and sheet metal wall separated the bake oven into two compartments for temperature control. The combination of NEG and ion pumps in the source chamber typically achieved pressures in the lower $10^{-11}$ Torr range, which led to very long photocathode dark lifetimes, with reactivations rarely necessary during normal use.

For photoemission, the photocathode was biased at $-268 \mathrm{~V}$ with respect to ground using a battery bias box, with photocurrent measured with a picoammeter [3.6] mounted serially between the bias box and ground. Figure 3.2 shows a SIMION [3.7] model of the electron beam trajectory though the source chamber, using typical bias voltages for each element, as noted, with the photocathode labeled "a" . To commission the polarimeter, photoemission was created using either a fixed (773 or $840 \mathrm{~nm}$ ) or variable $(\sim 770-780 \mathrm{~nm})$ diode laser, or using a monochromator, which produced un-collimated light from 700 to $850 \mathrm{~nm}$. Dual translation stages mounted beneath the vacuum chamber allowed movements of the laser beam across the photocathode, while maintaining normal incidence to its surface. An optical attenuator system was used to vary the laser power, and subsequently the photocurrent. Circularly polarized light was created using a quarter-wave plate immediately before the vacuum window leading to the source chamber. A computerized data acquisition program, written in LabVIEW [3.8], controlled a laser shutter, as well as an insertable half-wave plate, which was used to flip the helicity of the circularly polarized laser light. 


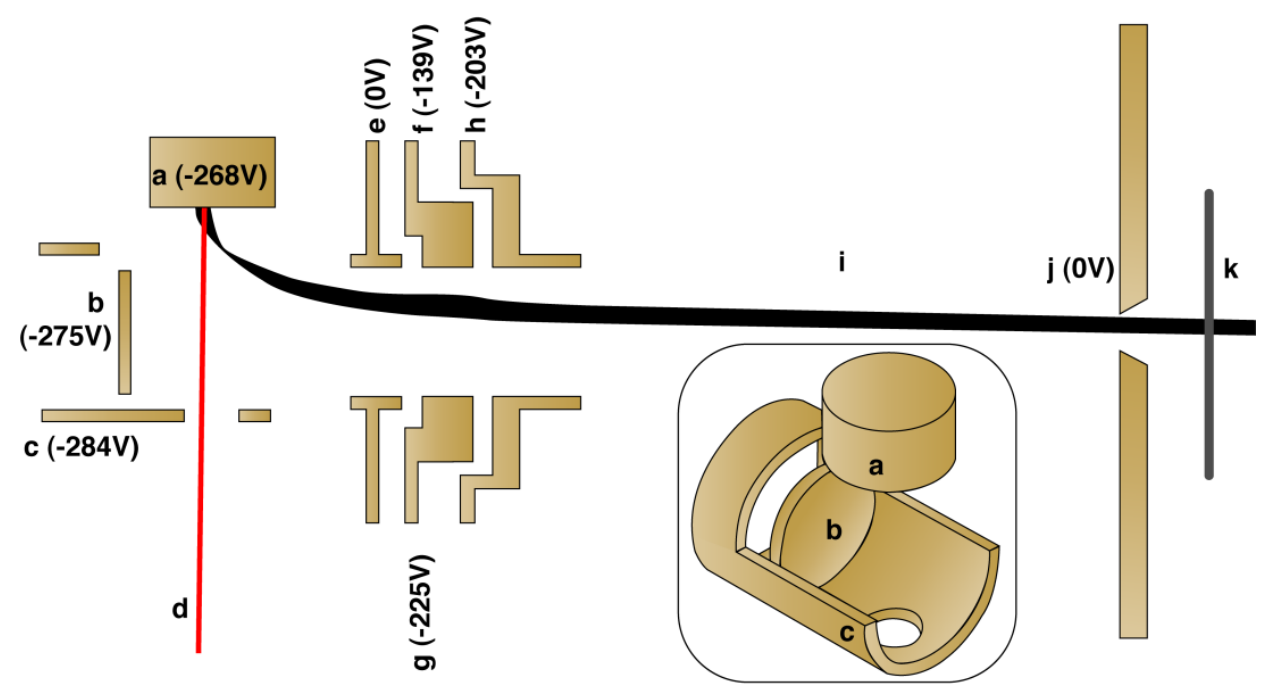

Figure 3.2: SIMION model of beam transport through the polarized electron source showing lenses and typical voltages in cross-section, with the three-dimensional inset showing detail of the photocathode and "pusher" electrostatic bend. The incident laser beam path and the SIMION modeled electron trajectories are labeled d and i, respectively. The beam limiting aperture is labeled $\mathrm{j}$, and $\mathrm{k}$ indicates the insertable planar electrode for current monitoring.

As noted previously, Mott scattering only detects asymmetries for transversely polarized electrons, while circularly polarized light produces longitudinally polarized electrons from GaAs. To rectify this mismatch, the beam direction was bent $90^{\circ}$ using an electrostatic bend (elements $\mathrm{b}$ and c) designed for other applications [3.9]. The beam was focused and steered to the Mott polarimeter chamber using one split lens (elements $\mathrm{f}$ and g) and two cylindrical electrostatic lenses (elements e and h) [3.10].

The polarized electron source vacuum chamber was then coupled to the Mott polarimeter though a transport section which was separated from the source chamber by a $3.2 \mathrm{~mm}$ diameter aperture (element $\mathrm{j}$ in Figure 3.2), which served to define the beam. An isolated planar electrode could be inserted after the aperture and before the Mott transport steering lenses, which were located $15 \mathrm{~cm}$ from the end of the electrostatic lenses in the source chamber. This electrode was used to monitor current that entered the transport system, and was used to confirm SIMION bias voltages to ensure proper steering of the electron beam in the beam transport section. 
CHAPTER 3: MOTT POLARIMETER

The Mott polarimeter, shown in Figure 3.3, was designed for ease of construction, and as such has no electrode structures except for the inner high-voltage hemisphere and grounded outer hemisphere, which supports simplified retarding-field grids. Its design differs from earlier designs [3.11] in that this design is smaller and simpler, in that it eliminates guard rings and ancillary electrodes. Electrons that entered the polarimeter were accelerated to energies from 5 to $50 \mathrm{keV}$ between two hemispherical stainless steel electrodes supported on a ceramic insulator (Figure 3.3). Electrons scattered from a gold target (5 microns of gold plated on a copper cylinder) inside the inner hemisphere. In principle, the target could be biased negatively relative to the inner hemisphere to suppress noise due to ions accelerated into the detectors [3.12]; however, this was not done, as no ion-related noise was observed. The vacuum chamber served as adequate radiation shielding at $30 \mathrm{kV}$ for typical operating currents up to $100 \mathrm{nA}$ on target. Scattered electrons decelerated in the gap between the inner and outer hemispheres and were detected with channel electron multipliers (CEMs) [3.12], each subtending 0.27 sr, centered at $120^{\circ}$. To reduce the chance of electrical discharge, the outer surface of the inner hemisphere was highly polished, and aperture holes in both hemispheres are rounded and polished. Two gold mesh [3.14] grids in front of each CEM, separated by $3.5 \mathrm{~mm}$, established a spatially well-defined retarding potential volume and rejected inelastically-scattered electrons. The grids were affixed to aluminum support rings using Aerodag [3.15] and isolated by ruby balls. As the retarding field increased negatively from ground, electrons that lost energy through inelastic scattering were increasingly excluded from the measurement, and when the retarding potential energy approached that of the incident beam kinetic energy, only the elastically scattered electrons were detected. For this dissertation, $\Delta \mathrm{E}$ will refer to the greatest energy a scattered electron can lose while still having been detected. Thus, for an incident beam with kinetic energy $\mathrm{K}$ and a retarding voltage on the grids equal to $\mathrm{V}, \Delta E=K-|e| V$. The two-grid retarder design has been found to provide better discrimination against inelastically scattered electrons at small values of $\Delta \mathrm{E}[3.16]$. 
Electrons were detected by two pairs of CEMs: the right/left pair was aligned to measure the Mott scattering asymmetry and the up/down pair could be used to measure any out-of-plane polarization due to physical mechanisms, instrumental asymmetries, or polarimeter misalignment. Electrostatic tube lenses and deflectors, also coated with Aerodag [3.15], steer and focus the incident electron beam into the polarimeter, as seen in Figure 3.1.

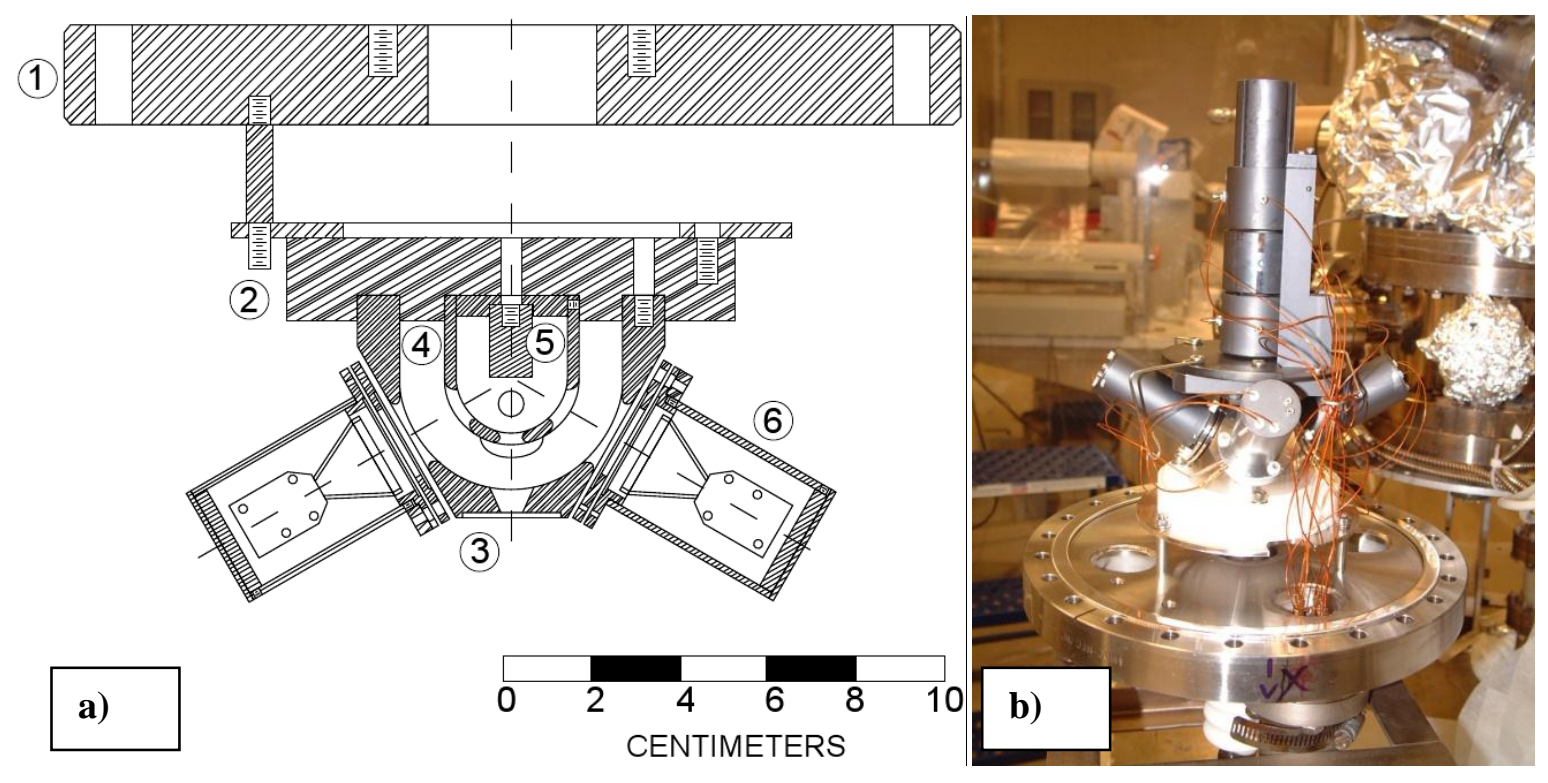

Figure 3.3: a) Scale cross section drawing of polarimeter showing: 1) 8 "Conflat $\AA$ mounting flange with $23 / 4$ " flange for high-voltage bushings and feedthroughs; 2) insulating standoff and mounting plate; 3) outer hemisphere; 4) highly polished stainless steel inner hemisphere; 5) target screwed into high voltage electrode; 6) channel electron multiplier in housing attached to retarding-field grid assembly. b) photograph of polarimeter with steering lenses attached.

\subsubsection{Mott Commissioning Methodology}

Many parameters have historically been used to characterize Mott polarimeters. Incident electron currents are often low, and as such, the polarimeter's detection efficiency, defined as the electron detection rate divided by the incident electron current, $\mathrm{I} / \mathrm{I}_{0}$, is important. A larger fraction of electrons can 
CHAPTER 3: MOTT POLARIMETER

be collected by increasing the effective solid angle subtended by the electron detectors at the Mott scattering target, which increases efficiency. One way to increase this angle is to decrease the size of the polarimeter. In addition, experiments requiring spin analysis of scattered electrons often place severe spatial constraints on the size of the polarimeters that can be used [3.3][3.17]. So-called "micro-Mott polarimeters," developed largely at Rice University by Dunning and co-workers over the last two decades [3.18][3.19][3.20][3.21], reduce polarimeter size to increase efficiency and ease of use. Like photocathodes, Mott polarimeters have a figure of merit that is used to determine their usefulness. This figure of merit, $\eta$, for a Mott polarimeter is inversely proportional to the square of the time required to measure polarization to a given statistical accuracy [3.17][3.22] and is defined as

$$
n=S_{e f f}^{2}\left(\frac{I}{I_{0}}\right)
$$

where $I / I_{0}$ is the afore mentioned efficiency and $S_{\text {eff }}$ is the effective Sherman function, as defined in Eq. 3.7.

In order to determine the efficiency of the Mott analyzer accurately, it was important to ensure that the measured signal pulses were associated with true target-scattered electron events, and that electronic dead time did not affect the result. Dead time issues were addressed by operating in the regime where count rates increased linearly with the incident beam current, and where efficiency was steady with current. This condition occurred for target currents less than $50 \mathrm{pA}$ at $5 \mathrm{kV}$ target bias, which corresponded to count rates less than $1 \mathrm{MHz}$ (Figure 3.4). The operating voltages for the channel electron multipliers (CEMs) were determined by both by finding the point where a $100 \mathrm{~V}$ increase in bias produced less than a $10 \%$ increase in count rate and by using an oscilloscope to ensure that the primary pulse peak height did not change across the same bias increase. The CEM high voltage bias boxes [3.23] are outside the vacuum chamber, and each channel was in a separate metal housing to reduce cross-talk. The 
capacitively-coupled CEM output signal was amplified with a pre-amp [3.24] placed immediately adjacent to the bias box.

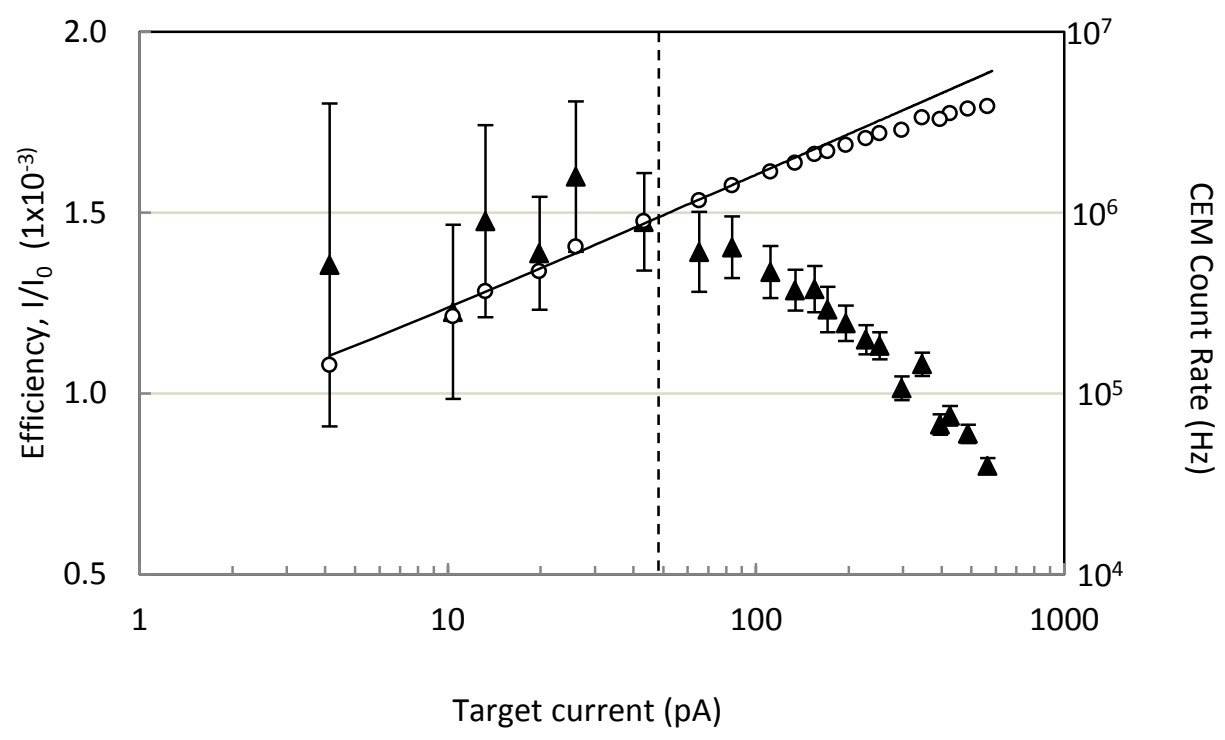

Figure 3.4: Efficiency $\left(\mathrm{I} / \mathrm{I}_{0}\right) v s$. target current at $5 \mathrm{keV}$ target bias and $\Delta \mathrm{E}=268 \mathrm{eV}(\boldsymbol{\Delta})$. Efficiency error bars are dominated by uncertainty in the current measurement. Channel electron multiplier (CEM) count rate (O) varies linearly for target currents below $100 \mathrm{pA}$ and rates below $1 \mathrm{MHz}$ while counting efficiency drops over 100pA or $1 \mathrm{MHz}$ (linear fit to data below $50 \mathrm{pA}$ and extended as a guide to the eye). Count rate was kept below $1 \mathrm{MHz}$ (vertical dotted line) during measurements to avoid electronic saturation effects.

The discriminator [3.25] threshold was determined by measuring both the asymmetry and signal-to-noise ratio as a function of threshold voltage with $\Delta E \approx 150 \mathrm{eV}$ to eliminate the high count rate from the scattered electrons with the largest energy losses. Figure 3.5 shows that discriminator thresholds of at least $250 \mathrm{mV}$ are needed for the signal/noise ratio and asymmetry to be independent of discriminator threshold; thresholds of $400 \mathrm{mV}$ were typically used during data acquisition. Peak pulse heights were typically over $1 \mathrm{~V}$ after amplification. The TTL pulses from the discriminators were counted via a LabVIEW DAQ program written for this purpose. 


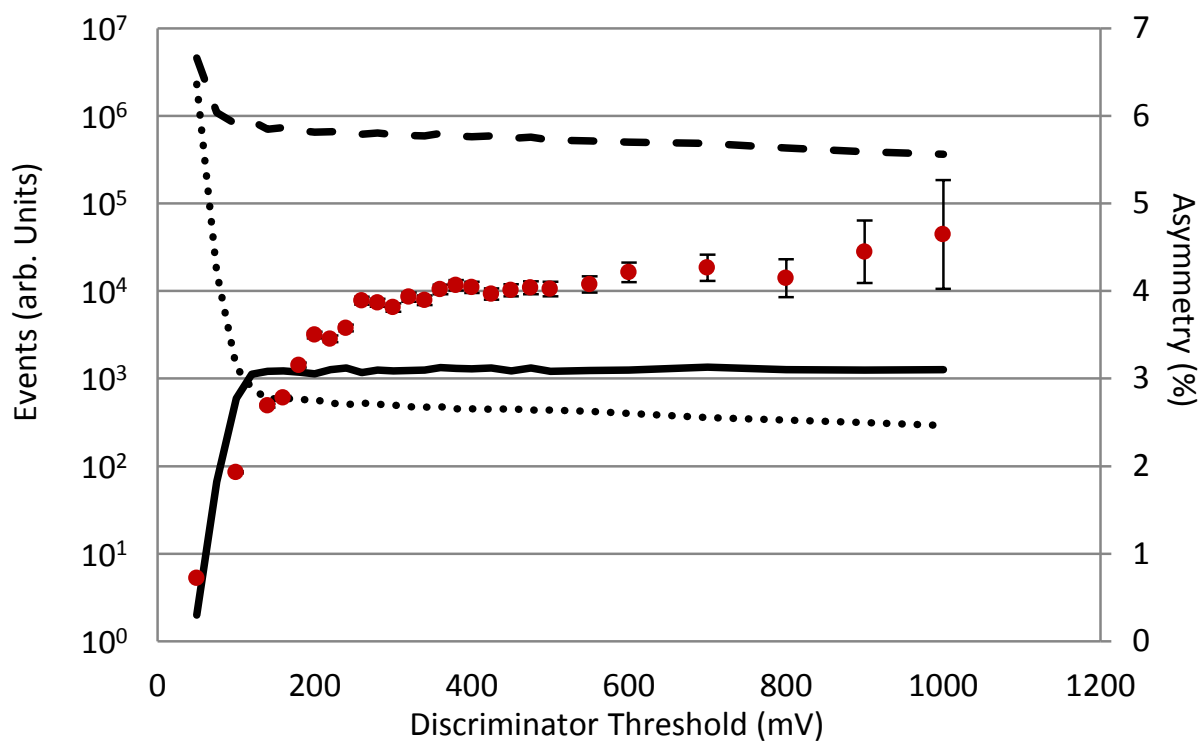

Figure 3.5: Asymmetry ( $\bullet$, right axis) and CEM count rate with beam on (dashed line, left axis) and beam off (dotted line, left axis) as a function of discriminator threshold. Ratio of beam-on to beam-off count rate is indicated by the solid line, left axis (see text). Data shown is for $5 \mathrm{kV}$ with superlattice photocathode and $773 \mathrm{~nm}$ laser illumination.

In order to determine the Sherman function of the polarimeter, the asymmetry of the scattered electrons was measured. As mentioned previously, GaAs emits electrons in one of two spin states depending on the handedness of the incident circularly polarized light, and this symmetry can be used to cancel instrumental asymmetries [3.3]. Measuring count rates in both the left (L) and right (R) detectors during both light helicity states, with the definitions $N^{+}=\sqrt{L_{1} R_{2}}$ and $N^{-}=\sqrt{L_{2} R_{1}}$, the measured asymmetry is then given by

$$
A=\frac{N^{+}-N^{-}}{N^{+}+N^{-}}
$$

where the subscripts on $L$ and $R$ refer to the count rates in detectors during the two different light polarization states. 


\subsection{Mott Commissioning Results}

\subsubsection{Efficiency}

Efficiency, $\mathrm{I} / \mathrm{I}_{0}$, was measured by first biasing the target at $+300 \mathrm{~V}$ and measuring the incident current with a picoammeter [3.6], then biasing the target at operating voltages up to $30 \mathrm{kV}$ and measuring CEM count rates. In order to ensure that electronic dead time issues were irrelevant for these measurements, the efficiency was measured as a function of target current with the target biased at $5 \mathrm{kV}$. Figure 3.6 shows that the efficiency is constant with current, at least up to a count rate of $500 \mathrm{kHz}$. Also shown in Figure 3.6 is the detected electron count rate as a function of target current; this relationship is linear up to $500 \mathrm{kHz}$, as expected in a regime with no dead time inefficiencies.

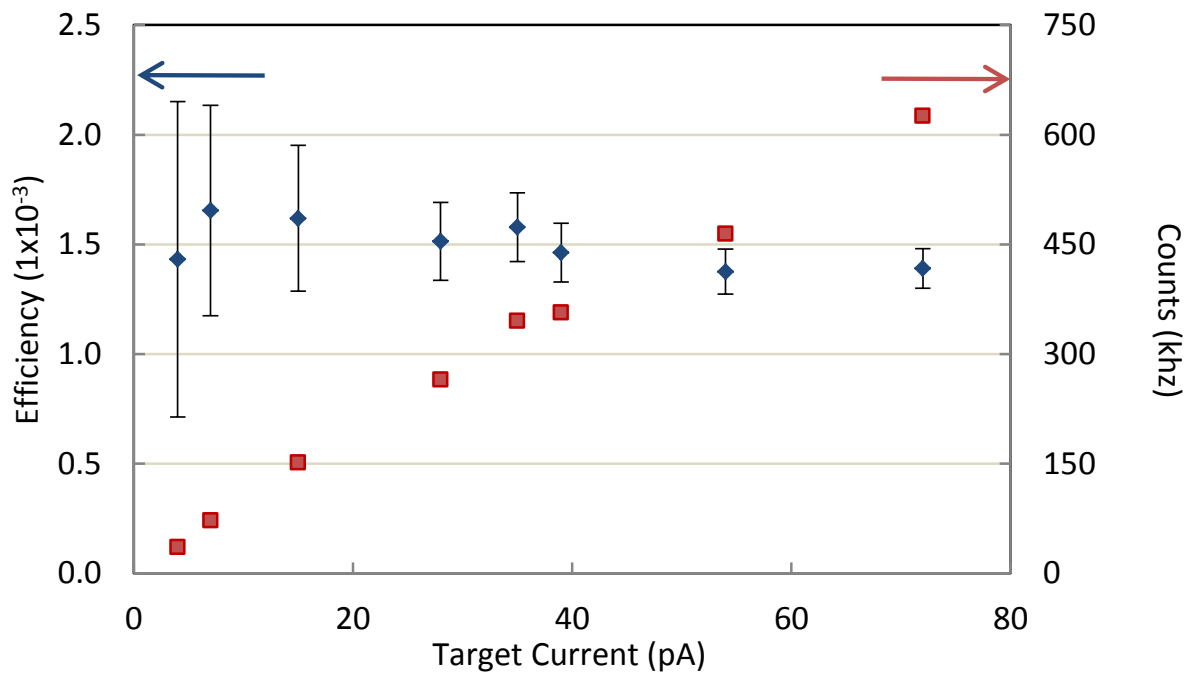

Figure 3.6: Mott efficiency ( $\diamond$, left axis) and CEM count rate ( $₫$, right axis) as a function of Mott target current. The target was biased at $5 \mathrm{kV}$.

Maximum efficiency, with essentially no rejection of inelastically-scattered electrons, is shown as a function of target bias in Figure 3.7a. The monotonic decrease of efficiency with increasing target voltage is a result of lowered electron scattering cross sections at higher incident energies. The efficiency was 
measured using the same polarized electron beam as is used for the asymmetry measurement, and was determined as a function of $\Delta \mathrm{E}$ for various target biases as shown in Figure 3.8a.

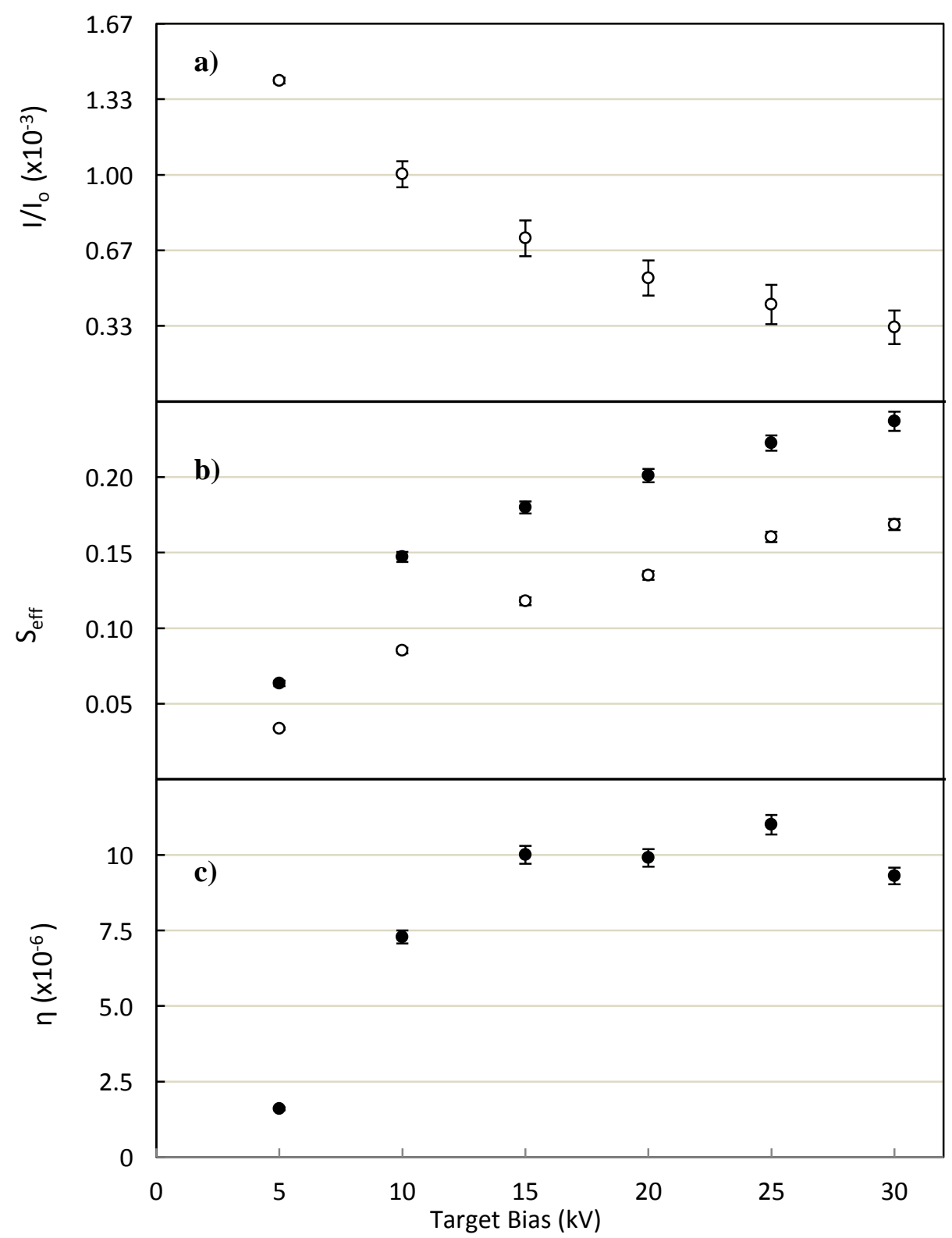

Figure 3.7: Variation as a function of target bias of the a) efficiency, $\mathrm{I} / \mathrm{I}_{\mathrm{o}}$ for $\Delta \mathrm{E}=268 \mathrm{eV}$; b) effective Sherman function for $\Delta \mathrm{E}=268 \mathrm{eV}(\mathrm{\circ})$ and extrapolated to $\Delta \mathrm{E}=0 \mathrm{eV}(\bullet)$; and c) the figure of merit, $\eta$, for $\Delta \mathrm{E}=268 \mathrm{eV}$. 


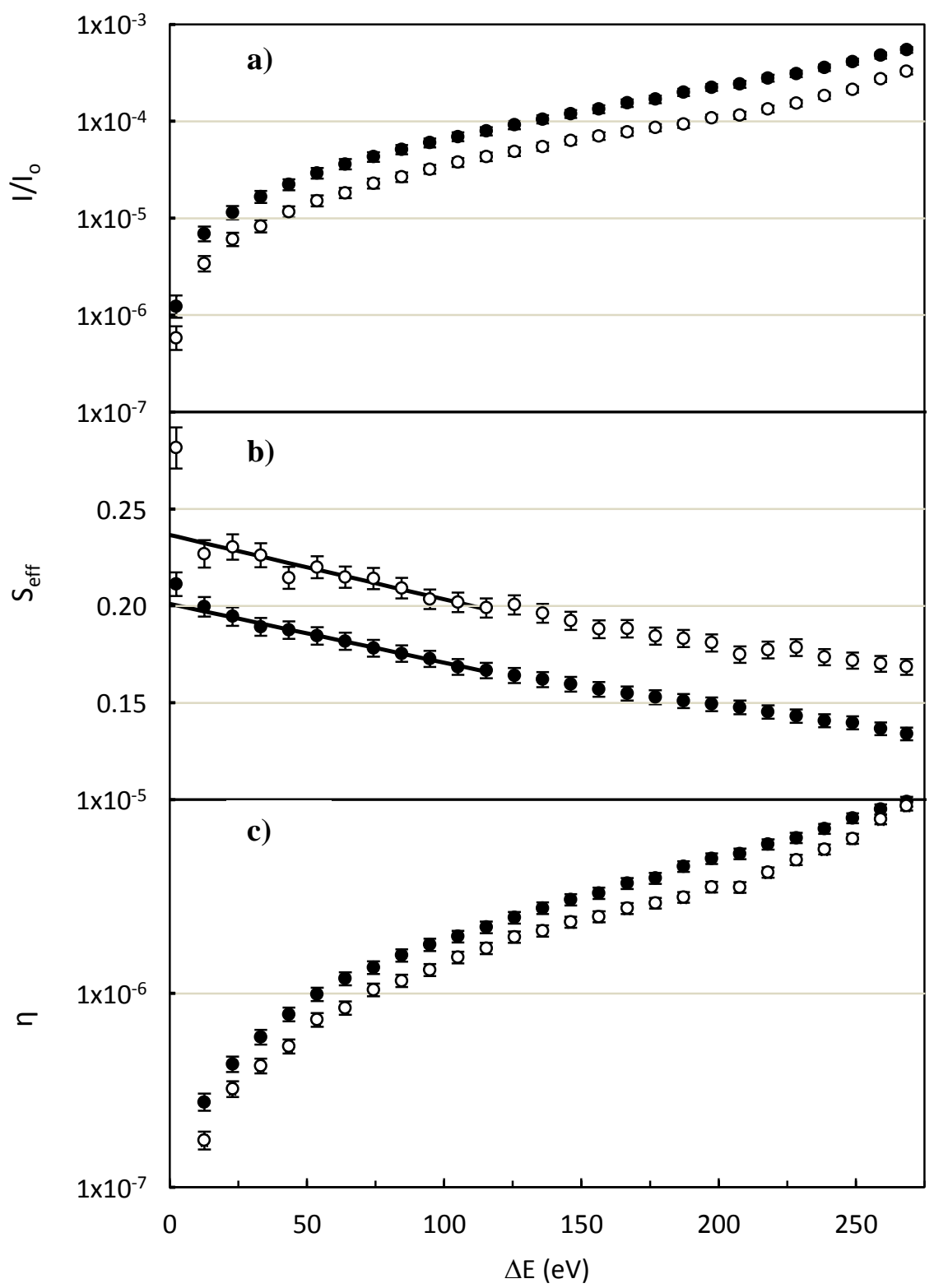

Figure 3.8 : Variation, as a function $\Delta \mathrm{E}$, of a) efficiency, $\mathrm{I} / \mathrm{I}_{\mathrm{o}} ; \mathrm{b}$ ) effective Sherman function, $\mathrm{S}_{\text {eff, with weighted }}$ linear fit for extrapolation to $\Delta \mathrm{E}=0 \mathrm{eV}$; and c) figure of merit, $\eta$. Target bias of $20 \mathrm{kV}(\bullet)$; Target bias of $30 \mathrm{kV}(\circ)$.

To verify that the target current at $300 \mathrm{~V}$ accurately represents target current at higher biases, current was measured as a function of target voltage using batteries up to $300 \mathrm{~V}$ and using a high voltage power supply [3.26] with nanoamp current sensitivity up to $7 \mathrm{kV}$ (see Figure 3.9). The slight increase in 
target current with target bias can be attributed to an increase in the number of secondary electrons produced at the target and upstream apertures that return to the target at higher bias.

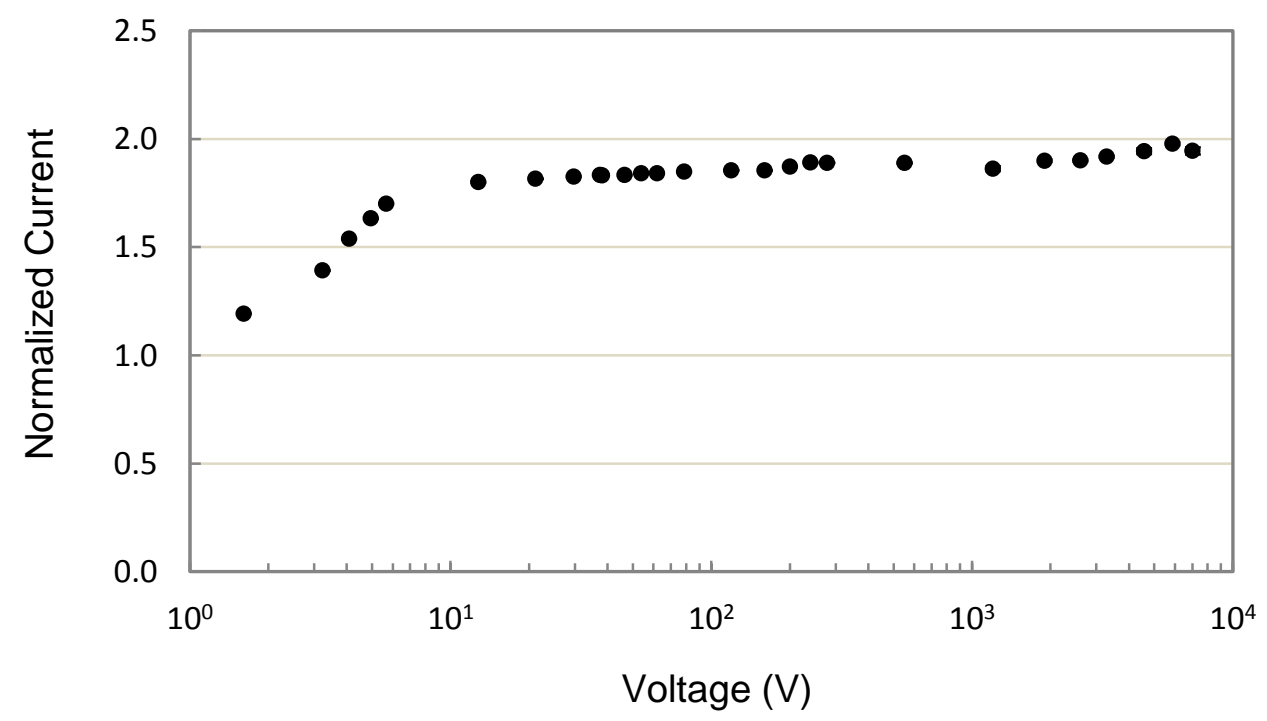

Figure 3.9: Target current as a function of target bias relative to the current measured with the target grounded, with currents typically on the order of $100 \mathrm{nA}$ (see text).

\subsubsection{Effective Sherman Function}

Taking advantage of prior CIS group work, the effective Sherman function, $S_{\text {eff, was determined }}$ by generating electron beam from photocathode material that is identical to the CEBAF polarized source [3.27][3.28],[3.29], and then dividing the measured asymmetry by the known beam polarization. These strained superlattice GaAs photocathodes [3.30], which consist of 14 pairs of layers of GaAs (4 nm) on GaAsP $(3 \mathrm{~nm})$, generate electron beams with polarization of $84 \%$ ( $\pm 1 \%$ statistical $\pm 1 \%$ systematic) when illuminated with $778 \mathrm{~nm}$ light, as measured by the CEBAF $5 \mathrm{MeV}$ Mott polarimeter [3.31] and corroborated by the four polarimeters in Jefferson Lab's three experimental halls. This value is reproducible across the photocathode diameter and between wafers of the same lot. The $S_{\text {eff }}$ vs. target bias is shown in Figure 3.7b. A linear weighted average fit of $S_{\text {eff }}$ versus $\Delta E$, using the range $\Delta E=0$ to 
CHAPTER 3: MOTT POLARIMETER

$115 \mathrm{eV}$, was used to determine $\mathrm{S}_{\text {eff }}$ for $\Delta \mathrm{E}=0$, as the count rates when $\Delta \mathrm{E}=0$ were too low to achieve satisfactory statistical accuracy. Figure $3.8 \mathrm{~b}$ shows both data and the linear fit for 20 and $30 \mathrm{kV}$ target bias. Backgrounds subtractions were made for both the residual rate with the light off and the residual rate when the retarding field exceeded the incident beam energy. The error bars reflect statistical errors in the asymmetry measurement as well as the $\pm 1 \%$ systematic and $\pm 1 \%$ statistical uncertainty (added linearly) in the CEBAF polarization measurement. The average Sherman function observed was $0.201 \pm$ 0.004 for $20 \mathrm{kV}$ and $\Delta \mathrm{E}=0$.

As a verification of the determination of $S_{\text {eff, }}$, polarization measurements were also made using bulk GaAs wafers diced from a single crystal, [3.32] and strained layer photocathodes with a single 100 $\mathrm{nm}$ thick GaAs layer grown on a lattice-mismatched substrate [3.33]. Figure 3.10 shows polarization measurements vs. target bias for the two materials, with data shown for several cycles of photocathode replacement and beam re-steering through the transport electron optics between cathode and target. The variation between nominally identical samples gives an estimate of the random systematic error of the measurements, which is then approximately $\pm 3 \%$ of the value. The polarization of electrons from strained GaAs measured at CEBAF is typically around $77 \%$, consistent with the measurements from this polarimeter. Because the measured polarization of bulk GaAs can vary widely depending on factors such as the substrate thickness and surface conditions, the measured polarizations near $30 \%$ for all target biases are within expectations, indicating consistency between the measured Sherman function and known polarization across many different samples. Also of note, is the consistency of polarization between different GaAs wafers of the same type, manufactured at approximately the same time, as this consistency confirms the technique of measuring $S_{\text {eff }}$ by comparing GaAs samples to those measured in the CEBAF tunnel. 


\subsubsection{Figure of Merit}

Figure $3.7 \mathrm{c}$ and Figure $3.8 \mathrm{c}$ show $\eta$ as a function of target bias and $\Delta \mathrm{E}$. Since $\mathrm{I} / \mathrm{I}_{\mathrm{o}}$ increases several orders of magnitude with $\Delta \mathrm{E}$ and the Sherman function decreases by less than a factor of two over the same range, the highest $\eta$ at is found at $\Delta \mathrm{E}=268 \mathrm{~V}$, corresponding to the incident beam energy. The measured $\eta$ was lower than that of comparable polarimeters, as a result of the previously noted decrease in efficiency, which outweighs the small increase in $S_{\text {eff }}$ of this design.

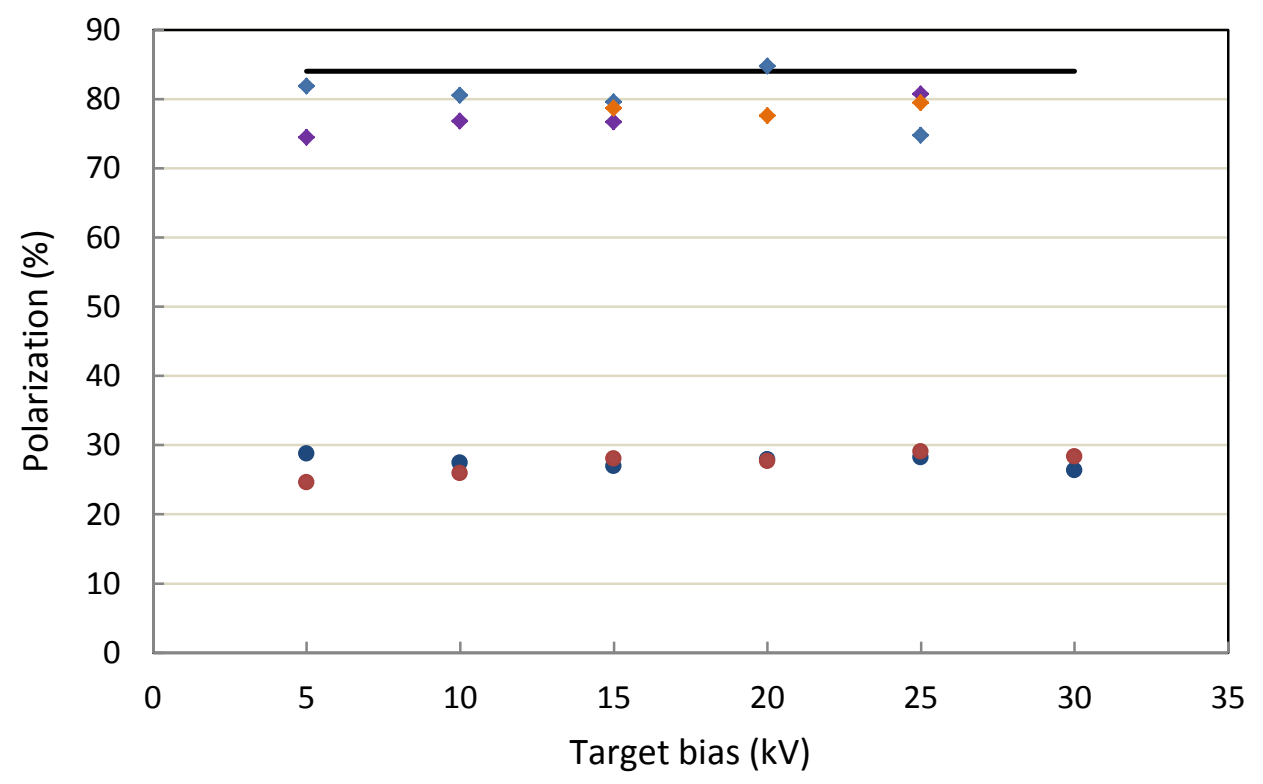

Figure 3.10: Measured electron polarization vs. target bias. Solid line indicates superlattice polarization of $84 \%$ used to determine $S_{\text {eff. }}$ Strained layer data: measured February $2008(\diamond)$, July $2008(\diamond)$, September $2008(\diamond)$. Bulk GaAs: measured September 2008 (•), June $2008(\bullet)$.

\subsection{MicroMott Polarimeter Conclusions}

A new type of simple micro-Mott polarimeter/polarized electron source system for photocathode characterization was commissioned, wherein the chief benefits are rapid sample changes, simplicity of construction, versatility of operation, and small size. Its operational range is 5 to $30 \mathrm{kV}$, which eliminates the radiation hazards that were present with JLab's previous offline polarimeter. The polarimeter's 
CHAPTER 3: MOTT POLARIMETER

analyzing power, or effective Sherman function, $\mathrm{S}_{\text {eff }}$, was calibrated through a comparison with Jefferson Lab's CEBAF $5 \mathrm{MeV}$ Mott polarimeter by measuring polarization from the same high-polarization photocathode material with both devices. The present design appears to have analyzing power and efficiency comparable to early designs of micro-Mott polarimeters (Table 3.1).In comparison with stateof-the-art designs, it has a comparable analyzing power, but significantly lower efficiency and subsequently figure-of-merit. This lower efficiency, which cannot be understood simply in terms of detector acceptance, is not a problem for this system, which is intended to characterize high-current photocathodes. In addition, currents on the Mott target are limited to only tens of nanoamp in order to ensure that there are no nonlinearities in the detected electron count rates. This compact, retarding-field Mott polarimeter, in combination with a polarized source, can be a valuable tool for off-line photocathode prequalification at CEBAF. In addition, the off-line polarimeter allows for novel photocathode polarization research, such as the electron polarization due to two-photon polarization studied in this dissertation.

Table 3.1: Comparison of various mini-Mott and micro-Mott designs at $20 \mathrm{kV}$ target bias with Au targets.

\begin{tabular}{|c|c|c|c|c|c|c|c|c|c|c|c|c|}
\hline \multirow[b]{2}{*}{ Ref. } & \multirow[b]{2}{*}{ Laboratory } & \multirow{2}{*}{$\begin{array}{c}\text { Max. } \\
\text { Seff }_{\text {ef }} \\
(\%)\end{array}$} & \multicolumn{3}{|c|}{ Max. } & \multicolumn{2}{|c|}{$\begin{array}{c}S_{\text {eff }} \text { at } \\
\text { max. }\end{array}$} & \multicolumn{3}{|c|}{$S_{\text {eff }}$ at } & & \multirow[b]{2}{*}{ Notes } \\
\hline & & & $\begin{array}{l}\Delta E \\
(e V)\end{array}$ & $\begin{array}{c}\mathrm{I} / \mathrm{I}_{0} \\
\left(10^{-4}\right)\end{array}$ & $\begin{array}{l}\Delta E \\
(e V)\end{array}$ & $\begin{array}{l}\mathrm{I} / \mathrm{I}_{0} \\
(\%)\end{array}$ & $\begin{array}{c}\text { Max. } \eta \\
\left(10^{-5}\right)^{\prime}\end{array}$ & $\begin{array}{l}\Delta \mathrm{E} \\
(\mathrm{eV})\end{array}$ & $\begin{array}{c}\max . \eta \\
(\%)\end{array}$ & $\begin{array}{l}\Delta \Omega \\
(\mathbf{s r})\end{array}$ & $\begin{array}{l}\text { Volume } \\
\left(10^{3} \mathrm{~cm}^{3}\right)\end{array}$ & \\
\hline [3.11] & Rice & 26 & 0 & 14 & 1300 & 12 & $\sim 2$ & 1300 & 12 & 0.02 & 8.5 & \\
\hline [3.21] & Rice & 23 & 400 & 53 & 1000 & 16 & 13 & 400 & 23 & 0.21 & 1.1 & $\mathrm{a}$ \\
\hline [3.20] & Rice & 21 & 300 & 94 & 1500 & 9 & 12 & 700 & 15 & 0.25 & 2.9 & b \\
\hline [3.19] & Rice & 11 & 1300 & $\sim 20$ & 1300 & 11 & 2.4 & 1300 & 11 & 0.11 & 2 & \\
\hline [3.18] & Rice & 11 & 1300 & 22 & 1300 & 11 & 2.7 & 1300 & 11 & 0.09 & 4.2 & \\
\hline [3.34] & Münster & 25 & 0 & & & & & & & 0.02 & 9.3 & \\
\hline [3.35] & Irvine & 20 & 500 & 6.7 & 1000 & 14 & 1.4 & 1000 & 14 & & & $\mathrm{c}$ \\
\hline [3.17] & Taiwan & 13 & 700 & & & & $\sim 2$ & & & 0.60 & & d \\
\hline [3.36] & Tokyo & 13 & 600 & 195 & 1400 & 10 & 18 & 1200 & 10 & 0.57 & 1.2 & \\
\hline [3.37] & St. Pet. & & & & & & 4.5 & & & 0.06 & 1.3 & e \\
\hline [3.38] & Edinburgh & 9 & 1300 & & & & & & & 0.06 & 2.2 & \\
\hline \multicolumn{2}{|c|}{ This work } & 20 & 0 & 5.4 & 268 & 13.5 & 1.0 & 268 & 13.5 & 0.27 & 1.4 & \\
\hline
\end{tabular}

a.Th target; $25 \mathrm{keV}$; max $\eta$ occurs over range of $\Delta \mathrm{E}$ from 400 to $1000 \mathrm{eV}$

b. Th target

c. U target

d. $23 \mathrm{keV}$ target bias

e.30 keV; refs. [3.20] and [3.21] indicate little change in $\eta$ between 20 and $25 \mathrm{keV}$ at $1300 \mathrm{eV}$ 


\section{GaAs: Polarization from Two-Photon Photoemission}

Polarized electron sources are important components of particle accelerators, like CEBAF at Jefferson Lab, where the spin of the polarized electron beam is used to study nuclear structure, the dynamics of strong interactions, electro-weak nuclear physics including parity-violation, and physics beyond the Standard Model [4.1]. The first GaAs-based polarized electron source used at an accelerator [4.2] provided beam polarization $\sim 35 \%$, with a theoretical maximum polarization limited to less than $50 \%$ [4.3],[4.4] due to the heavy-hole, light-hole energy level degeneracy of the ${ }^{2} \mathrm{p}_{3 / 2}$ valence band state (Figure 1a). Significantly higher beam polarization was obtained in the 1990s by introducing an axial strain within the GaAs crystal structure [4.5]-[4.7] which eliminates this degeneracy (Figure 4.1b). Today, beam polarization at accelerators routinely exceeds $80 \%$ using strained-superlattice GaAs/GaAsP structures [4.8],[4.9]. However, these high-polarization photocathodes are very expensive compared to unstrained bulk GaAs. In addition, because they are thin with respect to the photon absorption depth, the strained-superlattice photocathodes exhibit significantly lower quantum efficiency (QE) than bulk GaAs samples [4.7][4.10]. 
Conduction band

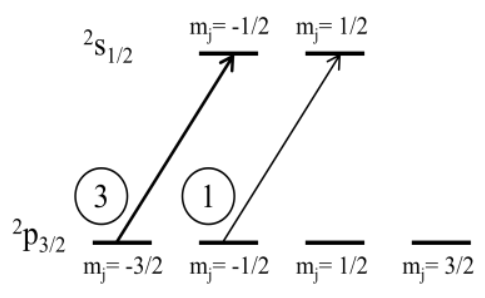

Valence band

(a)

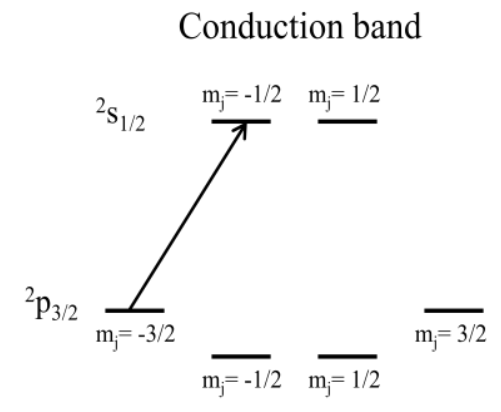

Valence band

(b)
Conduction band

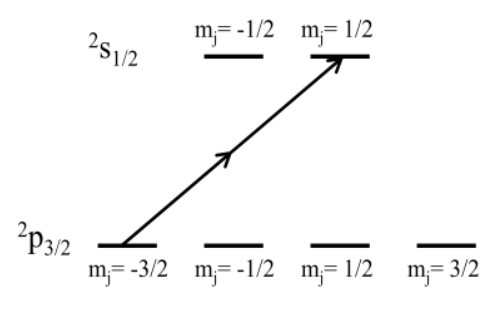

Valence band

(c)

Figure 4.1: Various means to populate the conduction band of GaAs with circularly-polarized light: a) one-photon excitation of unstrained GaAs, b) one-photon excitation of strained GaAs, and c) two-photon excitation of unstrained GaAs with photons having energy equal to half that of the band-gap. The circled values indicate relative transition probabilities for unstrained GaAs. Maximum theoretical polarization is 50\% from unstrained bulk GaAs via one-photon absorption, $100 \%$ from strained GaAs via one-photon excitation, and $-100 \%$ from unstrained GaAs via two-photon excitation, at least in the simple selection-rule picture of Ref. [4.12].

Two-photon absorption is a non-linear optical process [4.11] that occurs only within crystals that lack inversion symmetry, such as GaAs. Matsuyama et al. first proposed using this mechanism to obtain high polarization from unstrained GaAs [4.12]. They reasoned that quantum mechanical selection rules associated with the simultaneous absorption of two photons of circularly-polarized light at half the bandgap energy would provide a means to populate the conduction band with electrons of just one spin state (Figure 4.1c). The two-photon absorption probability is much less than that for conventional one-photon absorption. Nonetheless, the observation of beam polarization $>50 \%$ from unstrained bulk GaAs would be an exciting development, particularly for applications that do not require high beam current. Moreover, $1560 \mathrm{~nm}$ light, which is suitable for two-photon absorption, is now readily available because of its ubiquity in the telecommunications industry.

An experiment that relied on electron-hole photoluminescence measurements (but not photoemission) supported Matsuyama et al.'s idea, with electron-hole recombination fluorescence polarization measured to be $-58 \%$. This value was used to infer an electron polarization of $-95 \%$ at the time of excitation to the conduction band [4.13]. The sign of the two-photon polarization is an important 
CHAPTER 4: GaAs: POLARIZATION FROM TWO-PHOTON PHOTOEMISSION

feature of the Matsuyama et al., prediction. The negative polarization values denote a preferential population of electrons with opposite spin compared to that obtained using one photon absorption of light with the same circular polarization orientation. However, the simple selection rules invoked in Ref [4.12] ignore perturbation of local spherically-symmetric atomic fields by the neighboring atoms in the GaAs crystal lattice. Bhat et al. [4.14] showed that such perturbation of spherical symmetry becomes important for two-photon absorption. It enables "forbidden" intraband electron transitions to the conduction band via two-photon absorption in addition to transitions governed by the simple quantum mechanical selection rules considered by Matsuyama et al [4.12][4.13]. For "forbidden" intraband electron transitions, the photon angular momentum can be transferred to carrier orbital motion and the crystal structure, and not just the electron spin. Bhat et al. [4.14] predicted polarization via two-photon absorption to be comparable to conventional one-photon induced polarization, a value less than 50\%. Photoluminescence experiments using differential transmission pump/probe techniques indicated polarization equal to $48 \%$, in support of their predictions [4.14][4.15].

This Chapter presents a summary of the measurements made to test these ideas, which constitute the first direct observation of electron polarization resulting from two-photon excitation of GaAs. These measurements also allowed the study of the importance of electron depolarization in the photoemission process. The electron polarization for three GaAs sample thicknesses was determined using a compact retarding-field micro-Mott polarimeter. Two-photon absorption was verified by noting that quantum efficiency varied linearly with laser intensity, which was adjusted by different means. For each photocathode, the degree of spin polarization of the photoemitted beam was less than $50 \%$, contradicting the prediction of Matsuyama et al. [4.12]. Polarization via two-photon absorption was highest from the thin photocathode samples and comparable to that obtained via one-photon absorption $(\sim 43 \%)$. The two-photon absorption coefficient was measured to be $1.63 \pm 0.50 \mathrm{~cm} / \mathrm{GW}$, which is in reasonable agreement with previously cited results [4.16]. 


\subsection{Theoretical Background Information}

\subsubsection{Generation of Photoelectrons by One- and Two-Photon Absorption}

The structure of electron Bloch waves in semiconductors, $u_{n, k}(\vec{r}) \exp (i \vec{k} \vec{r})$, the product of a plane wave, $\exp (i \vec{k} \vec{r})$ and a periodic function, $u_{n, \boldsymbol{k}}(\vec{r})$, is the basis for understanding and interpreting our experiments. Here $\vec{k}$ is the electron wave vector in the reciprocal lattice and $\vec{r}$ is the electron spatial coordinate in the semiconductor. Substitution of the Bloch function into the Schrodinger equation yields an equation for the periodic part of the electron wave function in the unit cell:

$$
\left.\left\{\left[\frac{\vec{p}^{2}}{2 m_{0}}+V_{\text {cell }}(\vec{r})\right]+\left[\frac{\hbar}{m_{0}} \overrightarrow{(k} \cdot \vec{p}\right)+\frac{\hbar^{2} k^{2}}{2 m_{0}}\right]\right\} u_{n, \boldsymbol{k}}(\vec{r})=E_{n}(\vec{k}) u_{n, \boldsymbol{k}}(\vec{r}),
$$

where $V_{\text {cell }}(\vec{r})$ is the periodic crystal potential of the bulk GaAs and $\vec{p}$ is the momentum operator, $\vec{p}=-i \hbar \frac{\partial}{\partial \vec{r}}$. The wave vector, $\vec{k}$, is a parameter of Equation

(4.1), whose solution forms a complete set of periodic functions, $u_{n, \boldsymbol{k}}(\vec{r})$, and eigenvalues, $E_{n}(\vec{k})$, for each $\vec{k}$. Because of this completeness, any of these sets can be used as a basis for the expansion of other periodic functions in terms of $u_{n, \boldsymbol{k}}(\vec{r})$. The $\boldsymbol{k} \cdot \boldsymbol{p}$-method uses this feature for expanding $u_{n, \boldsymbol{k}}(\vec{r})$ and $E_{n}(\vec{k})$ in terms of the band-edge $(\vec{k}=0)$ Bloch functions, $u_{n, 0}(\vec{r})$, and eigenvalues, $E_{n}(0)$ [4.17]:

$$
u_{n, \boldsymbol{k}}(\vec{r})=u_{n, \mathbf{0}}(\vec{r})+\frac{\hbar}{m_{0}} \vec{k} \cdot \sum_{i \neq n} \frac{\vec{p}_{i n} u_{i, \mathbf{0}}(\vec{r})}{E_{n}(0)-E_{i}(0)}
$$

$$
\text { and } \quad E_{n}(\vec{k})=E_{n}(0)+\frac{\hbar^{2} k^{2}}{2 m_{0}}+\frac{\hbar^{2}}{m_{0}^{2}} \sum_{i \neq n} \frac{\left|\left\langle u_{i, 0}(\vec{r})|\vec{k} \vec{p}| u_{n, 0}(\vec{r})\right\rangle\right|^{2}}{E_{n}(0)-E_{i}(0)}
$$


CHAPTER 4: GaAs: POLARIZATION FROM TWO-PHOTON PHOTOEMISSION

Spherically-symmetric potentials of atoms composing the unit cell represent the main contribution to the crystal potential $V_{\text {cell }}(\vec{r})$ of the cell. Therefore, application of a symmetry hierarchy to $V_{\text {cell }}(\vec{r})$ is another useful tool for understanding the behavior of $u_{n, 0}(\vec{r})$ functions and for interpreting experimental results [4.18]. The symmetry hierarchy considers $V_{\text {cell }}(\vec{r})$ as a sum of a sphericallysymmetric potentials and lower-symmetry perturbations induced by relatively weak potentials of the outer atoms. In the first approximation of this approach, the band-edge Bloch functions, $u_{n, 0}(\vec{r})$, are eigenstates of the angular-momentum operator, $\boldsymbol{J}$. However, Equation (4.2) shows that although the $u_{n, 0}(\vec{r})$ are eigenstates of the angular momentum $\boldsymbol{J}$, the non-spherical potential of the outer atoms mixes all eigenstates in the cell when $\vec{k} \neq 0$. The periodic part of the Bloch function $u_{n, \boldsymbol{k}}(\vec{r})$ for $\vec{k} \neq 0$ is thus a superposition of angular momentum eigenstates. According to Equation (4.2), a larger value of $\vec{k}$ leads to stronger mixing, and the stronger this mixing, the weaker the angular momentum conservation in transitions involving such wave functions.

In the dipole approximation the rate of spin-polarized electron generation by circularly polarized light, $\vec{e}$, is proportional to the dipole transition matrix element squared, $\left|\left\langle u_{c, \boldsymbol{k}}(\vec{r})|\vec{e} \cdot \vec{p}| u_{v, \boldsymbol{k}}(\vec{r})\right\rangle\right|^{2}$, for both one- and two-photon absorption mechanisms. Substitution of the wave functions $u_{n, k}(\vec{r})$ in the matrix elements for Equation

(4.1) gives

$$
\left\langle u_{c, \boldsymbol{k}}|\vec{e} \cdot \vec{p}| u_{v, \boldsymbol{k}}\right\rangle=\vec{e} \cdot \vec{p}_{c v}+\frac{\hbar}{m_{0}} \vec{k} \cdot \sum_{i \neq c, v}\left(\frac{\vec{p}_{c i}\left(\vec{e} \cdot \vec{p}_{i v}\right)}{E_{c}(0)-E_{i}(0)}+\frac{\vec{p}_{i v}\left(\vec{e} \cdot \vec{p}_{c i}\right)}{E_{v}(0)-E_{i}(0)}\right)
$$

where $\vec{p}_{c v} \equiv\left\langle u_{c, 0}(\vec{r})|\vec{p}| u_{v, 0}(\vec{r})\right\rangle$ is the matrix element for an electron transition with $\vec{k}=0$. Recall that transitions calculated in the dipole approximation do not change electron spin-polarization [4.19] if spinorbit interaction is not taken into consideration. 
CHAPTER 4: GaAs: POLARIZATION FROM TWO-PHOTON PHOTOEMISSION

One can derive the selection rules for electron transitions from Equation (4.4). For instance, neglecting all but the conduction $(c)$ and valence $(v)$ bands, the second term on the right hand side of Equation (4.4) disappears so that it simplifies to $\left\langle u_{c, \boldsymbol{k}}|\vec{e} \cdot \vec{p}| u_{v, \boldsymbol{k}}\right\rangle=\vec{e} \cdot \vec{p}_{c v}$. Therefore, spin-polarized electron generation by one-photon absorption, which is proportional to $\left|\left\langle u_{c, \boldsymbol{k}}(\vec{r})|\vec{e} \cdot \vec{p}| u_{v, \boldsymbol{k}}(\vec{r})\right\rangle\right|^{2}$, is independent of $\vec{k}$ in the vicinity of $\vec{k}=0$ in the first approximation of the symmetry perturbation. On the other hand, generation by two-photon absorption is proportional to a product of $\left|\left\langle u_{c, \boldsymbol{k}}|\vec{e} \cdot \vec{p}| u_{v, \boldsymbol{k}}\right\rangle\right|^{2}$ with either $\left|\left\langle u_{c, \boldsymbol{k}}|\vec{e} \cdot \vec{p}| u_{c, \boldsymbol{k}}\right\rangle\right|^{2}$ or $\left|\left\langle u_{v, \boldsymbol{k}}|\vec{e} \cdot \vec{p}| u_{v, \boldsymbol{k}}\right\rangle\right|^{2}$. Because $\vec{p}_{c c}=\vec{p}_{v v}=0$, the latter two matrix elements vanish at $\vec{k}=0$, and thus the two-photon generation rate is proportional to $k^{2}$ for small $k$, and is quenched at the center $(\vec{k}=0)$ of the Brillouin zone. Similarly, a two-photon transition for a free atom between the considered levels would be forbidden due to the spin-parity selection rules. This makes the two-photon mechanism more sensitive to the mixing of different eigenstates of the angular momentum than the one-photon mechanism. In particular, spin-polarized electron generation near the band edge $(\vec{k}=0)$ by two-photon absorption is due to electron virtual transitions into the upper conduction $\left(c^{\prime}\right)$ band. Such a transition is proportional to the product of $\left|\left\langle u_{c^{\prime}, \boldsymbol{k}}|\vec{e} \cdot \vec{p}| u_{v, \boldsymbol{k}}\right\rangle\right|^{2}$ with $\left|\left\langle u_{c, \boldsymbol{k}}|\vec{e} \cdot \vec{p}| u_{c^{\prime}, \boldsymbol{k}}\right\rangle\right|^{2}$, which reduces to $\left|\left(\vec{e} \cdot \vec{p}_{c^{\prime} v}\right)\left(\vec{e} \cdot \vec{p}_{c c^{\prime}}\right)\right|^{2}$ in the first approximation of the symmetry perturbation and does not vanish at the zone center.

To summarize: the atomic structure of a GaAs crystal violates the spherical symmetry of the atomic fields that determine electronic states in semiconductors, which can lead to the non-conservation of angular momentum for $\vec{k} \neq 0$. However, the perturbation of the symmetry is very small for electrons in the center of the Brillouin zone, which is why the angular momentum is still a good quantum number for electron transitions induced by one-photon absorption when $\vec{k} \cong 0$. For transitions induced by twophoton absorption, the picture is more complicated, as spherical symmetry forbids such transitions at the center of the Brillouin zone. However, because of the perturbation of spherical symmetry due to the 
CHAPTER 4: GaAs: POLARIZATION FROM TWO-PHOTON PHOTOEMISSION

involvement of remote bands, there can be weak two-photon absorption in this region. Evidently, electron angular momentum cannot be conserved in transitions generated due to perturbation of spherical symmetry, such as those involved with two-photon absorption, which are features that Bhat et al. [4.14] took into account for two-photon absorption in solids. It is this conservation of angular momentum, or lack thereof, between the incident photon and excited electron that is at the heart of the maximum theoretical polarization due to two photon absorption. The aforementioned theoretical considerations, based on Ref. [4.14], preclude $100 \%$ polarization, while the work of Ref. [4.12] predicts a full -100 \% maximum polarization; this experiment was designed to differentiate between the two predictions, as well as the previous contradictory recombination fluorescence experiments [4.13][4.14].

\subsubsection{Two-Photon Quantum Efficiency}

Two-photon absorption at a depth $x$ from the surface is a non-linear effect proportional to the square of the beam intensity $I(x)$ at that location. Thus,

$$
d I / d x \cong-\alpha I-\beta I^{2}
$$

where $\alpha$ is the conventional one-photon absorption coefficient and $\beta$ is the two-photon absorption coefficient; higher-order absorptive processes have been neglected. Solving Eq. (4.5), we find

$$
I(x)=\alpha I_{0} \frac{\exp (-\alpha x)}{\alpha+\beta I_{0}(1-\exp (-\alpha x))}
$$

where $I_{0}=I(0)$ is the incoming beam intensity. Since Eqs. (4.5) and (4.6) describe the photoelectron generation rate, $G=-d I / d x$, the photoelectron diffusion equation, $D\left(\partial^{2} n / x^{2}\right)+G-n / \tau=0$, reduces to 
CHAPTER 4: GaAs: POLARIZATION FROM TWO-PHOTON PHOTOEMISSION

$$
D \frac{\partial^{2} n}{\partial x^{2}}+\frac{\alpha\left(I_{0} / \hbar \omega\right)}{1+\beta I_{0} x}+\frac{\beta\left(I_{0}^{2} / \hbar \omega\right)}{\left(1+\beta I_{0} x\right)^{2}}-\frac{n}{\tau}=0
$$

where $n / \tau$ is the photoelectron recombination rate, $n$ is the density of generated photoelectrons, $\tau$ is the photoelectron lifetime, $D$ is the diffusion coefficient, and $\hbar \omega$ is the photon energy so that $I_{0} / \hbar \omega$ is the flux of the incident photon beam [4.20]. Equation (4.7) describes the flow of electrons to the photocathode surface. Solution of this diffusion equation yields the quantum efficiency (QE):

$$
Q E=\left(S_{T} D \hbar \omega / I_{0}\right) \partial n(0) / \partial x
$$

where $S_{T}$ is the cathode transparency for photoelectrons that reach the surface. Assuming $S_{T}=1$, the photoelectron emission generated by one- and two-photon absorption reduces to

$$
\begin{aligned}
& Q E_{\omega}=-\frac{\alpha L}{\alpha L+1} \frac{1-\frac{\exp (-\alpha d)}{\cosh (d / L)}\left(1-\frac{\exp [(\alpha L-1) d / L]-1}{(\alpha L-1)}\right)}{1+\left(D / v_{T} L\right) \tanh (d / L)} \\
& \text { and } \quad Q E_{2 \omega}=\frac{\beta I_{0} L \tanh (d / L)}{1+\left(D / v_{T} L\right) \tanh (d / L)^{\prime}}
\end{aligned}
$$

respectively, wherein $d$ is the sample thickness, $L$ is the photoelectron diffusion length, and $v_{T}$ is the average thermal velocity with which electrons escape from the cathode into vacuum. Assuming $S_{T}$ is the same for both one- and two-photon emission, the ratio of these QEs is proportional to the beam intensity $I_{0}$ but insensitive to the $v_{T}$ :

$$
\frac{Q E_{2 \omega}}{Q E_{\omega}}=\left(\frac{\beta}{\alpha}\right) I_{0} \frac{(\alpha L+1) \tanh \left(\frac{d}{L}\right)}{1-\frac{\exp (-\alpha d)}{\cosh \left(\frac{d}{L}\right)}\left(1-\frac{\exp \left[(\alpha L-1) \frac{d}{L}\right]-1}{(\alpha L-1)}\right)} .
$$

For a bulk sample with $\alpha d \gg 1$ and $d \gg L$, Equations (8), (9) and (10) reduce, respectively, to 


$$
\begin{gathered}
Q E_{\omega}=-\frac{\alpha L}{\alpha L+1} \frac{1}{1+\left(D / v_{T} L\right)}, \\
Q E_{2 \omega}=\frac{\beta I_{0} L}{1+\left(D / v_{T} L\right)}, \\
\text { and } \quad \frac{Q E_{2 \omega}}{Q E_{\omega}}=(\alpha L+1)\left(\frac{\beta}{\alpha}\right) I_{0} .
\end{gathered}
$$

On the other hand, Equation (4.11) yields $Q E_{2 \omega} / Q E_{\omega}=(\beta / \alpha) I_{0}$ for the thin samples that $\alpha d \ll 1$ and $d \ll L$, which is independent of the film thickness but proportional to the incoming beam intensity.

\subsubsection{Electron Polarization via One- and Two- Photon Absorption}

The theoretical limits of spin-polarization for photoelectrons generated by one- and two-photon absorption are, respectively, $P_{\omega 0}=50 \%$ [4.3] and either $P_{2 \omega 0}=-100 \%$ [4.12] or $P_{2 \omega 0}=50 \%$ [4.14], as discussed above. However, these limits do not account for depolarizing effects encountered during electron transport through, and then out of, the GaAs. This depolarization is also described by the diffusion equation similar to Eq. (4.7), where now $n=n_{s p}$ is the density of spin-polarized photoelectrons and $\tau=\tau_{s p}$ is the photoelectron spin-relaxation time. Solution of this diffusion equation yields for polarization $P$ of emitted photoelectrons:

$$
P=\left(S_{T P} / S_{T}\right)\left(\partial n_{s p}(0) / \partial x\right) /(\partial n(0) / \partial x)
$$


CHAPTER 4: GaAs: POLARIZATION FROM TWO-PHOTON PHOTOEMISSION

where $S_{T P}$ is the probability of spin conservation during emission from cathode for spin-polarized photoelectrons that reach the surface. The diffusion equation shows that spin relaxation during the photoelectron diffusion to the photocathode surface reduces the polarization of emitting photoelectrons generated by one-photon and two-photon absorption from $P_{\omega 0}$ and $P_{2 \omega 0}$ to the net spin polarizations $P_{\omega}$ and $P_{2 \omega}$, respectively. Assuming $S_{T P}=1$, the net spin polarization of emitted photoelectrons reduces to

$$
\begin{gathered}
P_{\omega}=P_{\omega 0} \frac{l_{s p}}{L} \frac{\alpha L+1}{\alpha l_{s p}+1} \frac{1-\frac{\exp (-\alpha d)}{\cosh \left(d / l_{s p}\right)}\left(1-\frac{\exp \left[\left(\alpha l_{s p}-1\right) d / l_{s p}\right]-1}{\left(\alpha l_{s p}-1\right)}\right)}{1-\frac{\exp (-\alpha d)}{\cosh (d / L)}\left(1-\frac{\exp [(\alpha L-1) d / L]-1}{(\alpha L-1)}\right)} \frac{1+\frac{D}{v_{T} L} \tanh (d / L)}{1+\frac{D}{v_{T} l_{s p}} \tanh \left(d / l_{s p}\right)} \\
\text { and } \quad P_{2 \omega}=P_{2 \omega 0} \frac{l_{s p} \tanh \left(d / l_{s p}\right)}{L \tanh (d / L)} \frac{1+\left(D / v_{T} L\right) \tanh (d / L)}{1+\left(D / v_{T} l_{s p}\right) \tanh \left(d / l_{s p}\right)},
\end{gathered}
$$

where $l_{s p}$ is the photoelectron spin relaxation length in photocathode, $l_{s p}=\sqrt{D \tau_{s p}}$. Noteworthy, the polarization of emitting photoelectrons is insensitive to the beam intensity. The ratio of these polarizations is

$$
\frac{P_{2 \omega}}{P_{\omega}}=\frac{P_{2 \omega 0}}{P_{\omega 0}} \frac{\left(\alpha l_{s p}+1\right) \tanh \left(d / l_{s p}\right)}{(\alpha L+1) \tanh (d / L)} \frac{1-\frac{\exp (-\alpha d)}{\cosh (d / L)}\left(1-\frac{\exp [(\alpha L-1) d / L]-1}{(\alpha L-1)}\right)}{1-\frac{\exp (-\alpha d)}{\cosh \left(d / l_{s p}\right)}\left(1-\frac{\exp \left[\left(\alpha l_{s p}-1\right) d / l_{s p}\right]-1}{\left(\alpha l_{s p}-1\right)}\right)}
$$

For the bulk sample, again with $\alpha d \gg 1, d \gg L$, and $d \gg l_{s p}$, Equations (4.16) - (4.18) reduce, respectively, to

$$
P_{\omega}=P_{\omega 0} \frac{l_{s p}}{L} \frac{\alpha L+1}{\alpha l_{s p}+1} \frac{1+\left(D / v_{T} L\right)}{1+\left(D / v_{T} l_{s p}\right)}
$$




$$
\begin{gathered}
P_{2 \omega}=P_{2 \omega 0} \frac{l_{s p}}{L} \frac{1+\left(D / v_{T} L\right)}{1+\left(D / v_{T} l_{s p}\right)}, \\
\text { and } \frac{P_{2 \omega}}{P_{\omega}}=\frac{P_{2 \omega 0}}{P_{\omega 0}} \frac{\alpha l_{s p}+1}{\alpha L+1} .
\end{gathered}
$$

On the other hand, for thin films, when $\alpha d \ll 1$ and $d \ll l_{s p}$, the dependence on the thickness $d$ disappears in the polarization ratio given in Equation (4.18):

$$
\begin{array}{r}
P_{\omega}=P_{\omega 0} \frac{1+\left(D / v_{T} L\right)(d / L)}{1+\left(D / v_{T} l_{s p}\right)\left(d / l_{s p}\right)}, \\
P_{2 \omega}=P_{2 \omega 0} \frac{1+\left(D / v_{T} L\right)(d / L)}{1+\left(D / v_{T} l_{s p}\right)\left(d / l_{s p}\right)^{\prime}}, \\
\text { and } \\
\frac{P_{2 \omega}}{P_{\omega}}=\frac{P_{2 \omega 0}}{P_{\omega 0}} .
\end{array}
$$

Equation (4.24) shows that with the experimental measurement of $\frac{P_{2 \omega}}{P_{\omega}}$ as a function of film thickness, one can extract the initial polarization ratio $\frac{P_{2 \omega 0}}{P_{\omega 0}}$ for verification of the theory developed in Ref. [4.14], for which $\frac{P_{2 \omega 0}}{P_{\omega 0}}=1$. 


\subsection{Experimental setup}

The apparatus, which was the same as that used in Chapter 3, consisted of a low-voltage polarized electron source chamber for installing and activating photocathodes, a beam transport section, and a micro-Mott retarding-field polarimeter (Figure 3.1) Unstrained bulk GaAs was mounted on a long stalk that could be lowered into the source chamber, and the GaAs was then activated using standard practice [4.19] to negative electron affinity (NEA) with $\mathrm{Cs}_{\text {and }} \mathrm{NF}_{3}$. The photocathode was biased at $-268 \mathrm{~V}$ using batteries and the emitted electron beam was delivered to the micro-Mott polarimeter using a $90^{\circ}$ electrostatic deflector [4.22] and electrostatic steering lenses [4.23]. The beam transport system and the micro-Mott polarimeter are described more thoroughly in Chapter 3, as well as in another publication [4.19].

Two laser wavelengths were used: 778 and $1560 \mathrm{~nm}$ for one- and two-photon absorption, respectively. Optical systems for each wavelength could be quickly and reproducibly moved in and out of position beneath the vacuum chamber. These systems comprised numerous optical elements manufactured for the appropriate wavelength, including optical attenuators, linear polarizers, quarterwave plates, and insertable half-wave plates. While the $1560 \mathrm{~nm}$ laser system was in place (Figure 4.2), long-pass optical filters (two at $1350 \mathrm{~nm}$ and one at $850 \mathrm{~nm}$ ) were inserted into the laser path to ensure that no light below $850 \mathrm{~nm}$ could enter the vacuum apparatus and illuminate the photocathode. These filters were needed because fiber amplifiers (described below) produce a small amount of light in the visible and near-IR wavelengths via second harmonic generation. Even a small amount of undetected light striking the photocathode in the visible or near-IR wavelength range could easily overwhelm any beam produced at $1560 \mathrm{~nm}$, where two-photon photocurrent is comparatively small. Insertion of additional $1350 \mathrm{~nm}$ long-pass filters had no effect on the amount of photocurrent, indicating that only $1560 \mathrm{~nm}$ light reached the photocathode. Finally, a mirror system mounted to dual translation stages 
directed the laser beam vertically into the source chamber and provided a means to map the quantum efficiency of the photocathode (see below).

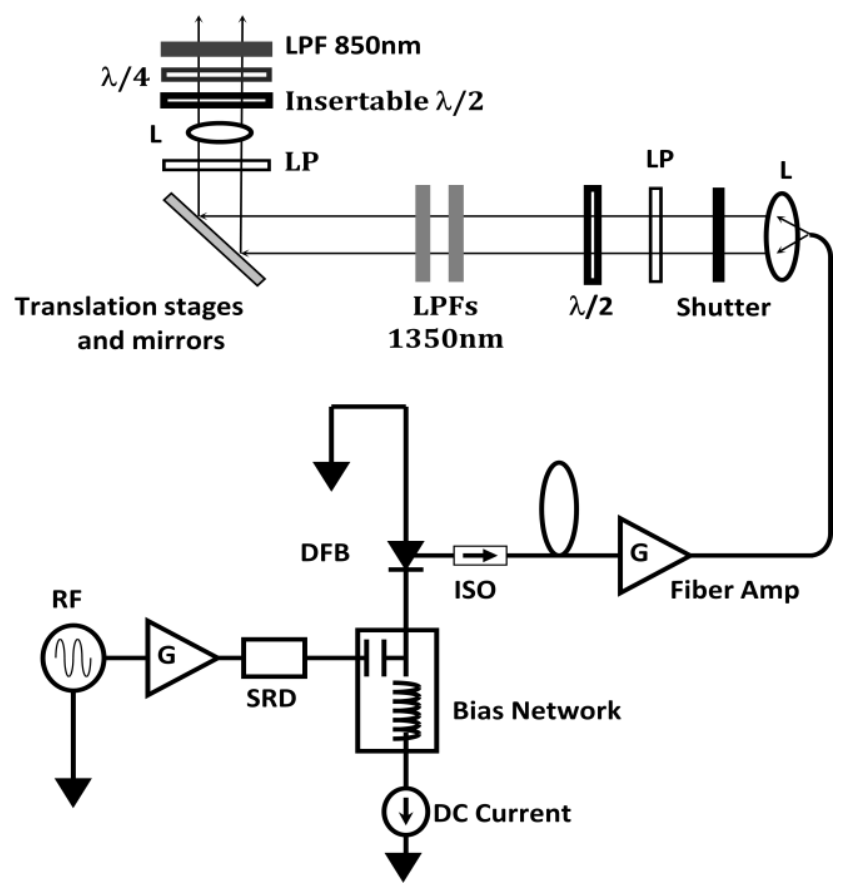

Figure 4.2: Schematic of the $1560 \mathrm{~nm}$ light source used to generate a two-photon photo-emitted electron beam from un-strained bulk GaAs showing DFB, distributed feedback Bragg reflector diode laser; ISO, fiber isolator; SRD, step recovery diode; L, lens; LP, linear polarizer; $\lambda / \mathbf{2}$ and $\lambda / \mathbf{4}$, half-wave and quarter-wave plates; LPF, long pass filter.

The $778 \mathrm{~nm}$ light source was a simple low-power diode laser that could be operated either in DC or in RF pulsed-mode via gain-switching at repetition rates from 250 to $1000 \mathrm{MHz}$. The QE at $778 \mathrm{~nm}$ for one-photon absorption should be independent of incident laser intensity, and thus $Q E_{\omega}$ should be independent of average power, peak power, or laser spot size.

The $1560 \mathrm{~nm}$ laser consisted of a gain-switched fiber-coupled diode "seed" laser and fiber-amplifier (Figure 4.2), that produced up to $5 \mathrm{~W}$ average power at repetition rates from 250 to $2000 \mathrm{MHz}$, with optical pulse widths of $\sim 40$ to $60 \mathrm{ps}$, depending on the rate [4.24]. By using short-pulse light, high peak power was obtained to enhance the two-photon absorption process. For two-photon absorption, the QE at $1560 \mathrm{~nm}$ should vary proportionally with laser intensity. In this experiment, there were three "knobs" 
CHAPTER 4: GaAs: POLARIZATION FROM TWO-PHOTON PHOTOEMISSION

used to vary the intensity at the photocathode: the average power of the light, the spot size of the laser on the photocathode, and the pulse repetition rate (while keeping pulse widths similar and average power constant). These parameters affect intensity at the photocathode:

$$
\begin{gathered}
\mathrm{I}_{\mathrm{avg}}=\mathrm{P}_{\mathrm{avg}} / \mathrm{A} \\
\text { and } \quad I_{\text {peak }}=\frac{I_{\text {avg }}}{\mathfrak{D}}=\frac{P_{\text {avg }}}{A \cdot \mathfrak{D}}=\frac{P_{a v g}}{A \cdot t \cdot f},
\end{gathered}
$$

where $P_{\text {avg }}$ is the average power of the laser, $\mathfrak{D}$ is the duty factor, which is the product of the optical pulse width $t$ and the laser pulse repetition rate $f$, and $A$ is the spot size of the laser on the photocathode.

Variation in laser light intensity was obtained by changing the average power of the incident laser light using an optical attenuator, by changing the pulse repetition rate, and by altering the size of the laser beam at the photocathode. The optical attenuator was able to deliver up to $2 \mathrm{~W}$ average power and could attenuate this to less than $70 \mathrm{~mW}$. Different laser spot sizes were obtained by using lenses of different focal length. A $30 \mathrm{~cm}$ focal-length lens in the optical train below the vacuum window was used to produce a beam focal waist of $270 \mu \mathrm{m}$ FWHM at the photocathode; longer focal-length lenses placed in the same location produced larger beam waists, up to 1,200 $\mu \mathrm{m}$ FWHM. Laser spot size measurements were made using a knife edge scan in two directions. The mirrors on the translation stage were removed in order to place the scanner system at an equivalent distance to the photocathode from the lenses. Because changing lenses in the optical system could steer the beam, QE scans (see below) were done after each lens change and after each light source switch in order to ensure direct comparisons of measurements.

The rf-pulsed nature of the laser systems provided a means to easily vary the peak intensity of the light at the photocathode. The optical pulse width of the gain-switched laser system remained 
approximately constant between 40 and 60 ps FWHM across the range of pulse repetition rates. The fiber amplifier provided the same output power for each repetition rate, so the peak intensity of the laser system could be varied by changing the pulse repetition rate, with the highest intensity obtained at lower pulse repetition rates.

The unstrained GaAs wafers were activated, and reactivated as needed, by heating to $\sim 550{ }^{\circ} \mathrm{C}$ to clean the surface and by then applying $\mathrm{Cs}$ and $\mathrm{NF}_{3}$ to make a NEA surface [4.25]. The thick sample $(625 \mu \mathrm{m})$ was epi-ready unstrained bulk GaAs, with a (100) surface, p-doped with Zn of density $\sim 5 \times 10^{18} / \mathrm{cm}^{-3}$. The thin samples $(0.18$ and $0.32 \mu \mathrm{m})$ were grown via MOCVD with $\mathrm{p}$-doping of $\mathrm{Zn}$ (density $\sim 4 \times 10^{18} / \mathrm{cm}^{-3}$ ) on thick GaAs substrates, with an intervening barrier layer of $\mathrm{p}-\mathrm{Al}_{0.3} \mathrm{Ga}_{0.7} \mathrm{As}$ that was $\sim 0.9 \mu \mathrm{m}$ thick. The band gap of this barrier layer is much larger than that of GaAs, which ensured that no electrons were created in the barrier layer from the $780 \mathrm{~nm}$ or $1560 \mathrm{~nm}$ light and also that any electrons excited in the substrate material did not reach the photocathode surface.

Several steps were taken to ensure that the surface state of the photocathode was the same for the pairs of measurements comparing the one- and two-photon processes. Each set of QE and polarization data was taken during the same photocathode activation. However, the Cs deposition at the photocathode was non-uniform, which caused the $\mathrm{QE}$ of the GaAs wafer to vary over its surface. The QE was measured using a picoammeter in series with the biased cathode and ground, using the formula (based in Eq. (2.1))

$$
Q E \%=\frac{124 \cdot I}{P \cdot \lambda}
$$

in which $I$ is the photocurrent in $\mu \mathrm{A}, P$ is the laser power in $\mathrm{mW}$, and $\lambda$ is the wavelength of the incident light in $\mathrm{nm}$. Using the $\mathrm{x}-\mathrm{y}$ translation stages underneath the vacuum window, "QE scans" were performed by measuring the $\mathrm{QE}$ across the cathode. Also, because of the simple design of the electrostatic lenses used to bend the electron beam $90^{\circ}$ from the photocathode to the Mott target, the amount of beam 
CHAPTER 4: GaAs: POLARIZATION FROM TWO-PHOTON PHOTOEMISSION

transmission from the photocathode to the polarimeter was not constant across the photocathode. By connecting another picoammeter to the Mott target biased at $\sim+300 \mathrm{~V}$ while performing a QE scan, a map of photocurrent transmission from the cathode to the polarimeter could also be made. The highest transmission, corresponding to about $20 \%$ of the photoemitted beam striking the polarimeter target, occurred for emission from a specific location of the GaAs photocathode about $2 \mathrm{~mm}$ in diameter, whereas the entire activated photocathode was $\sim 12 \mathrm{~mm}$ in diameter. The area of highest transmission and that of highest QE were located roughly $6 \mathrm{~mm}$ apart. Unless otherwise noted, QE data is presented at the location of highest $\mathrm{QE}$ of the photocathode, and polarization data was always taken at the highest transmission location of the photocathode.

\subsection{Results and Discussion}

\subsubsection{One- and Two-Photon Quantum Efficiency}

The $625 \mu \mathrm{m}$ thick unstrained bulk GaAs was first evaluated using $778 \mathrm{~nm}$ light at different repetition rates with varying average powers. As expected, $Q E_{\omega}$, remained fairly constant versus input power and pulse repetition rates (Figure 4.3a). Because of the range of powers used, the abscissa in Figure 4.3a is plotted logarithmically. Also, note the break in the scale of the mantissa from zero. There was a slight decrease in $Q E_{\omega}$ as the laser power increased for a given repetition rate, which can be attributed to the surface charge limit effect due to the relatively large peak current that was extracted up to $\sim 800 \mu \mathrm{A}$ [4.26]. In addition, a decrease in $Q E_{\omega}$ observed with increasing intensity (lower repetition rates) at a given average power can be explained by the space charge effects of the shorter electron bunches [4.26]. 
The QE behavior at $1560 \mathrm{~nm}$ (Figure 4.3b) was qualitatively different from the behavior at 778 $\mathrm{nm}$. Indicative of a two photon-absorption process, higher light intensity produced higher $Q E_{2 \omega}$, which increased linearly with power and intensity. Using the $1560 \mathrm{~nm}$ source, a maximum photocurrent of $\sim 5$ $\mathrm{nA}$ was measured at $250 \mathrm{MHz}$ and $1.9 \mathrm{~W}$. It should be noted that high average power $(>\sim 2 \mathrm{~W})$ caused the photocathode to warm up; over a long time the stalk holding the photocathode reached a steady state temperature of $\sim 70^{\circ} \mathrm{C}$. Over several minutes, this heat significantly degraded the photocathode $Q E_{2 \omega}$, as has been seen in other experiments, which can be attributed to evaporation of the CsF layer from the photocathode surface [4.27]. To help preserve the $Q E_{2 \omega}$ performance, cold nitrogen gas was applied to the back surface of the photocathode stalk. However, even with this cooling, the $Q E_{2 \omega}$ decayed over time at the highest powers of each set of measurements. Because of this decay, these measurements do not provide suitable comparisons of the $Q E_{2 \omega}$ change with varying laser intensity amongst different repetition rates. However, the demonstrated linear increase with power for each individual repetition rate provides clear evidence for a two-photon photoemission process. To further ensure that the $Q E_{2 \omega}$ decay did not influence the results, it was measured at the same repetition rate while holding the average power constant at either 0.8 or $1.3 \mathrm{~W}$. Again, $Q E_{2 \omega}$ was proportional to the laser intensity. 

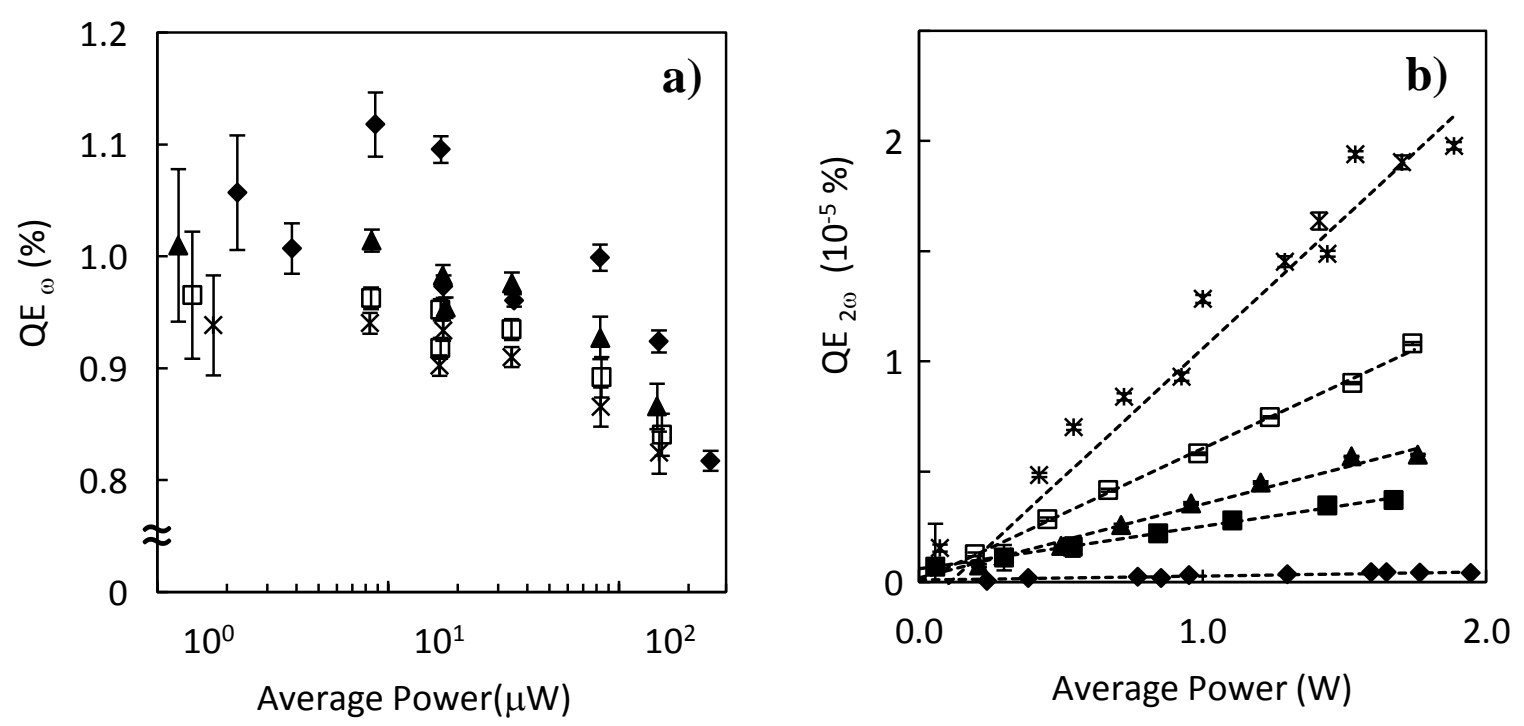

Figure 4.3: a) Representative $\mathrm{QE}_{\omega}$ vs. input power (on a logarithmic scale) at $778 \mathrm{~nm}$, for the laser pulse repetition rates given below, and b) representative $\mathrm{QE}_{2 \omega}$ at $1560 \mathrm{~nm}$.. Dashed lines are linear fits to data for a given repetition rate. The repetition rate legend is the same for both plots: $\mathrm{DC}(\downarrow), 2000 \mathrm{MHz}(\mathbf{\bullet}), 1000 \mathrm{MHz}$ (४), $500 \mathrm{MHz}(\square)$, and $250 \mathrm{MHz}(*)$. Figure 4a does not include $2000 \mathrm{MHz}$ data.

Next, the bulk GaAs was evaluated using $778 \mathrm{~nm}$ and 1560 light at different repetition rates while holding the average power constant. As Eq. (4.26) shows, changing the repetition rate while holding the average power and the pulse width constant will change the peak intensity of the incident laser. As the exact size of the laser spot was not known for this set of measurements, only the peak power is known. Because the spot size did not change throughout the measurement, peak power can be substituted for peak intensity with regards to the expected $\mathrm{QE}$ behavior of one- and two-photon absorption; $Q E_{\omega}$ should be constant and $Q E_{2 \omega}$ should be linear with regards to peak power. Again, as expected, $Q E_{\omega}$ was constant with regards to the peak power (Figure $4.4 \mathrm{a}$ - note the beak in the scale of the mantissa from zero). The QE behavior at $1560 \mathrm{~nm}$ (Figure 4.4b) was again qualitatively different from the behavior at $778 \mathrm{~nm}$, and as expected, $Q E_{2 \omega}$ increased linearly with peak power. This behavior is further indication that the photoemission resulting from the $1560 \mathrm{~nm}$ light was due to two-photon absorption. 

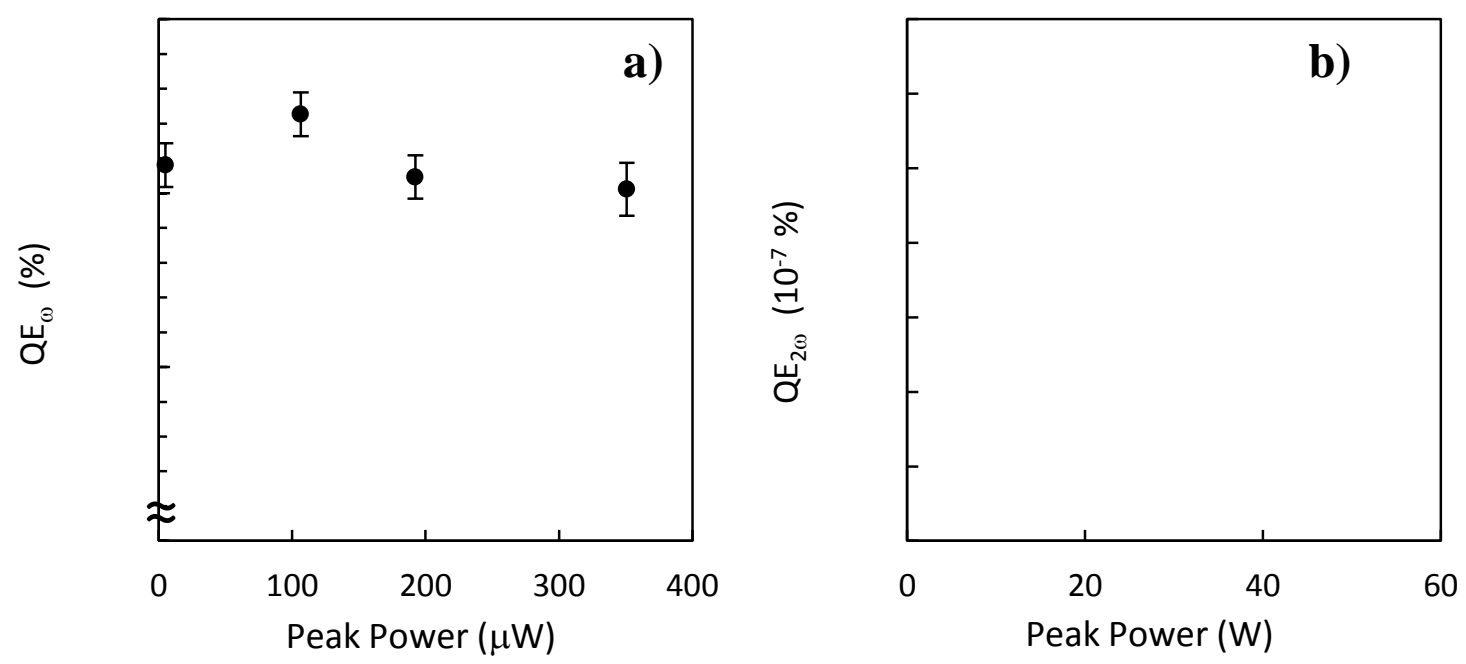

Figure 4.4: a) Representative $\mathrm{QE}_{\omega}(\bullet)$ vs. peak power at $778 \mathrm{~nm}$, with an average power of $5 \mu \mathrm{W}$ and b) representative $\mathrm{QE}_{2 \omega}(\circ)$ vs. peak power at $1560 \mathrm{~nm}$, with an average power of $0.8 \mathrm{~W}$. To vary peak power, the laser repetition rate was varied while holding the average power and pulse width constant.

As a last qualitative check on the two-photon QE behavior, while using a pulse repetition rate of $250 \mathrm{MHz}$, the laser intensity was varied by changing the size of the laser spot at the photocathode, with the $Q E_{2 \omega}$ results shown in Figure 4.5. As noted above, the photocathode activation was not uniform across the surface, causing the location of maximum QE to not be coincident with the location of maximum beam transmission to the polarimeter. QE scans were performed, and comparative measurements with both wavelengths were always made at the same photocathode location. The measurements in Figure 4.5 show results from both the maximum QE ( $\mathbf{a})$ and the QE at the maximum transmission locations ( $\mathbf{\Delta})$. At $778 \mathrm{~nm}$ (Figure 4.5a), the QE was nearly constant versus peak intensity, with any deviation attributable to sampling the non-uniform surface with the different size laser spot sizes. Because the maximum $\mathrm{QE}$ was found at the very edge of the photocathode, sampling the location with a smaller, more intense, laser spot had the effect of slightly raising the measured QE. The smaller laser spots were fully incident on the photocathode, while the larger spots were incident also on an electrostatic bend electrode. The behavior at $1560 \mathrm{~nm}$ was quite different from that at $778 \mathrm{~nm}$, as $Q E_{2 \omega}$ increased linearly with laser peak intensity (Figure 4.5b), as expected from Eq. (4.10). Here again, the 
two-photon process is shown qualitatively to be the dominant mechanism in the absorption of $1560 \mathrm{~nm}$ light in GaAs. The many qualitative demonstrations of pure two-photon absorption at $1560 \mathrm{~nm}$ were needed to ensure that no light at higher energy than the band-gap, such as $778 \mathrm{~nm}$, was present.
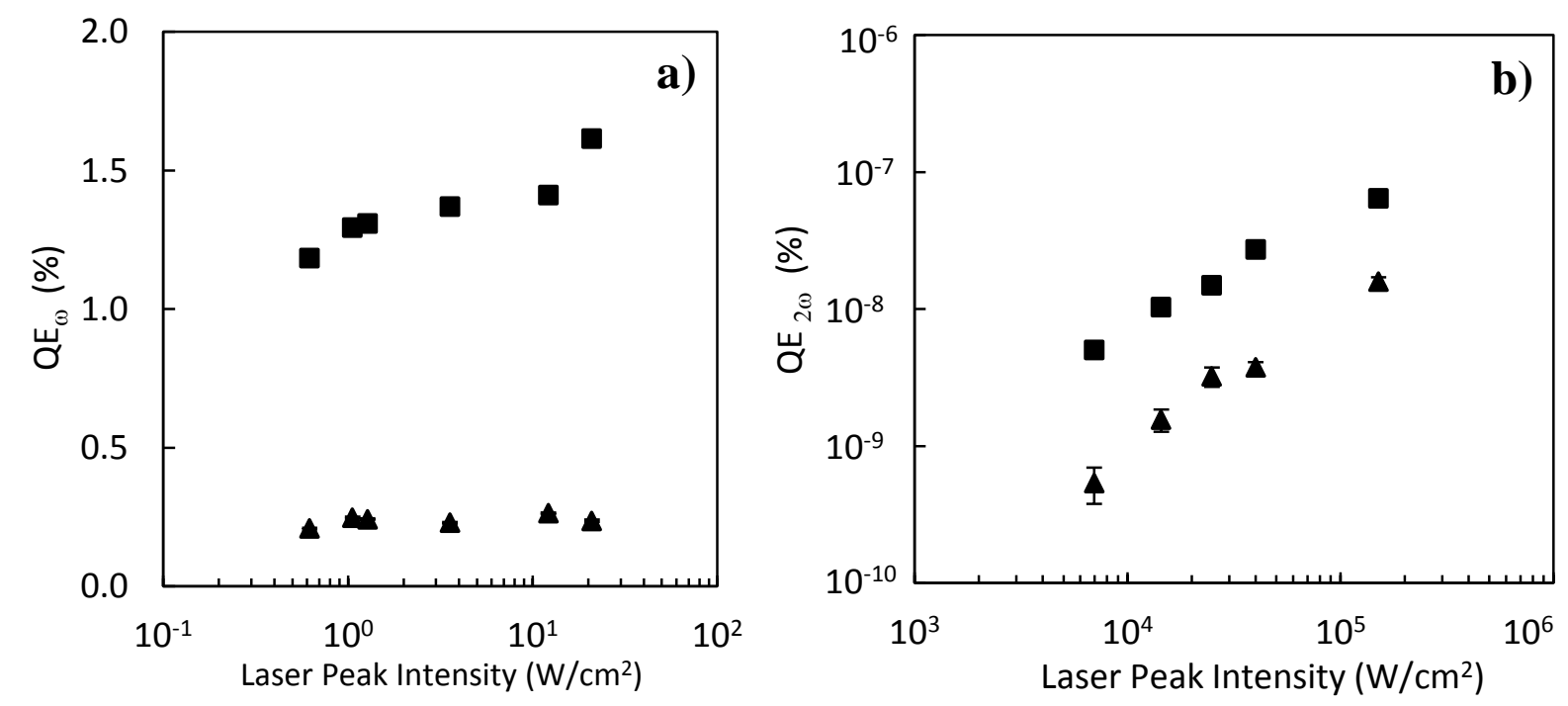

Figure 4.5: QE vs. peak intensity of the laser beam caused by changing the laser spot size at the cathode. The QE across the photocathode was not uniform: Maximum QE (-) and maximum transmission location QE ( $(\mathbf{\Delta})$ at the photocathode location for maximum transmission to the polarimeter (see text). a) QE at $778 \mathrm{~nm}$. b) QE at $1560 \mathrm{~nm}$. Data were obtained using a laser repetition rate of $250 \mathrm{MHz}$.

For quantitative verification of the two-photon absorption effect, the measurements of $\mathrm{QE}$ as a function of laser peak intensity were used to evaluate the two-photon absorption coefficient, $\beta$. Solving Eq. (4.14) for $\beta$, gives

$$
\beta=\frac{Q E_{2 \omega}}{Q E_{\omega}} \cdot \frac{1}{I_{0}} \cdot \frac{\alpha}{\alpha \cdot L+1}
$$

Figure 4.6 shows $\beta$ measurements as function of laser peak intensity for three different surface regions of the photocathode: the position corresponding to maximum $\mathrm{QE}$, the position corresponding to maximum transmission to the polarimeter target, and the center of the photocathode. These $\beta$ values assume an effective electron diffusion length $L=1.5 \cdot 10^{-4} \mathrm{~cm}$ [4.28] and a one-photon absorption coefficient $\alpha=1.05 \cdot 10^{4} \mathrm{~cm}^{-1}$ [4.29]. As expected, $\beta$ is independent of laser peak intensity, and is consistent across 
the photocathode, even though the QE is non-uniform. From all three sets of data, a weighted average of $\beta=1.6 \pm 0.5 \mathrm{~cm} / \mathrm{GW}$ can be calculated. It should be noted that a theoretical value of $\beta=7 \mathrm{~cm} / \mathrm{GW}$ at $1550 \mathrm{~nm}$ has recently been reported [4.16]. The difference in these values is probably caused by the different dopant and defect concentrations, as the theoretical model assumed carrier concentrations at least a factor of ten lower than the tested GaAs dopant level. The difference in wavelength may also contribute to this disparity. In addition, it should also be noted that the photoemission of electrons is a complicated process due to surface effects, such as electron reflection, and these effects have not been fully accounted for in the formulation of $\beta$ used in Eq. (4.28).

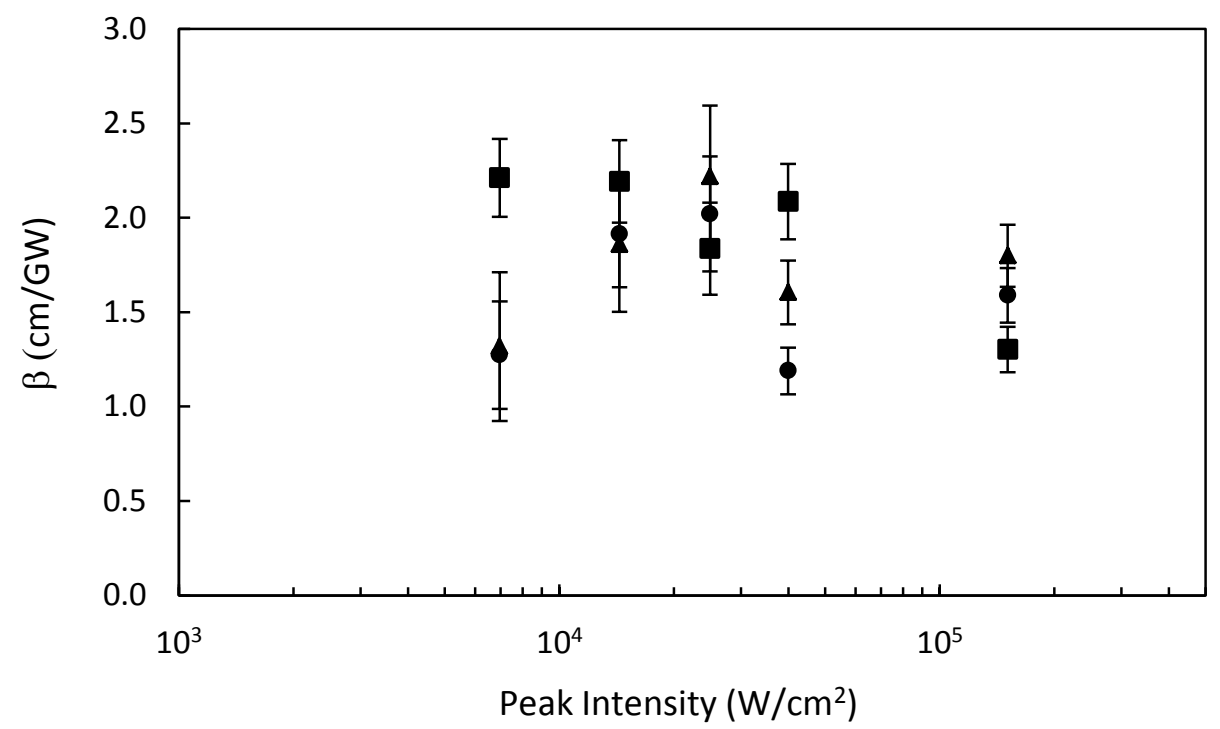

Figure 4.6: Coefficient $\boldsymbol{\beta}$ of two-photon absorption as a function of the laser peak intensity, varied by changing size of the laser focus at the GaAs surface, with $250 \mathrm{MHz}$ pulse repetition rate for three locations of the photocathode: at the position corresponding to maximum QE (ש), at the position corresponding to maximum transmission to the polarimeter target $(\boldsymbol{\Delta})$, and at the photocathode center $(\bullet)$.

\subsubsection{Electron Polarization from Thick $(625 \mu \mathrm{m})$ Unstrained GaAs}

The polarization asymmetry, A, of the electron beams generated with both one- and two-photon excitation was measured using the micro-Mott polarimeter, in the manner described in [4.19] with the 
CHAPTER 4: GaAs: POLARIZATION FROM TWO-PHOTON PHOTOEMISSION

target biased at $20 \mathrm{kV}$ and with a maximum electron energy loss in the target $\Delta \mathrm{E}=0$. The quantity $\Delta \mathrm{E}=0$ is the greatest energy loss an electron scattered by the Mott target can have suffered and still be detected. The electron polarization was determined using the known value of $0.201 \pm 0.005$ for the effective Sherman function, $\mathrm{S}_{\mathrm{eff}}$, of the polarimeter and the Mott polarimetry equation, $P_{e}=A / S_{\text {eff }}$. Repeated measurements indicated that the polarization at both $1560 \mathrm{~nm}$ and $778 \mathrm{~nm}$ was stable with regard to extracted photocurrent, which ensured that no electronic dead time issues were present at the experimental count rates. The polarization at $778 \mathrm{~nm}$ was $33.4 \pm 0.8 \%$, which is typical of bulk GaAs. At $1560 \mathrm{~nm}$, the measured polarization was $16.8 \pm 0.4 \%$, which was significantly lower than any prediction, even when accounting for depolarization effects similar in magnitude to the photoelectrons at $778 \mathrm{~nm}$. Importantly, the sign of the beam polarization obtained via two-photon absorption was the same as that obtained via one-photon absorption.

Because of the unexpectedly low polarization at $1560 \mathrm{~nm}$, additional measurements were made to ensure that proper optical procedures were used. The effect of the amount of laser polarization on beam polarization is shown in Figure 4.7. The quarter-wave plate was rotated to vary the degree of circular polarization of the incident light. As expected, the electron polarization for both one- and two-photon excitation varied sinusoidally with the degree of circular polarization. This behavior of the electron polarization with the degree of light polarization indicates that there was no issue with the optical arrangement that systematically affected the electron polarizations. 


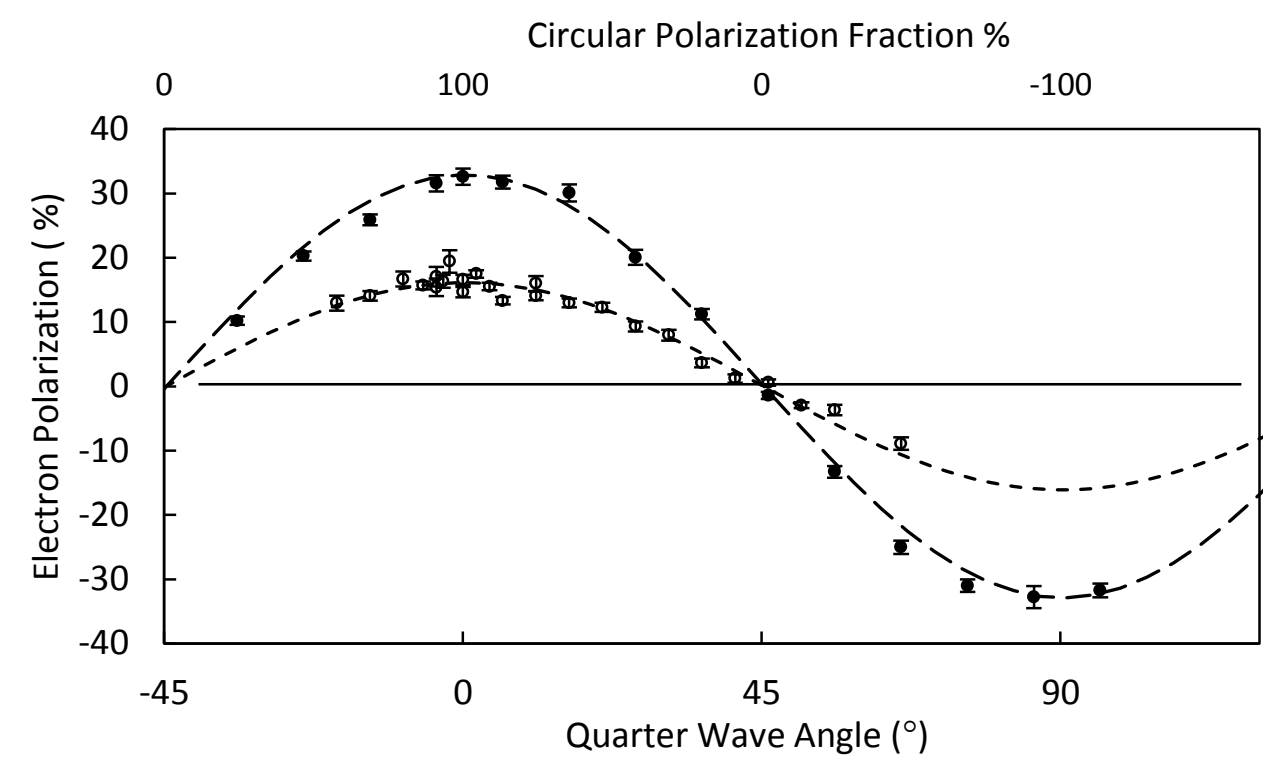

Figure 4.7: The polarization of the photoelectron beam from bulk GaAs at $778 \mathrm{~nm}(\bullet)$ and at $1560 \mathrm{~nm}(\circ)$ as a function of the orientation of the quarter wave plate relative to the zero angle corresponding to $100 \%$ circularly polarized light. The dashed lines represent sinusoidal fits to the data.

\subsubsection{Electron Polarization from Thin Unstrained GaAs}

One reason for the lower-than-expected value of polarization associated with two-photon emission is that $1560 \mathrm{~nm}$ light has a much larger absorption depth in GaAs. This is apparent when one compares $\beta \cdot I_{\max }=2.5 \cdot 10^{-4} \mathrm{~cm}^{-1}$ for typical values of $I_{\max }$ we used in our experiment with $\alpha=$ $1.03 \cdot 10^{4} \mathrm{~cm}^{-1}$. This leads to longer exit paths of the electrons, and hence more depolarization. As Eq. (4.17) is dependent on some initial value of polarization, $P_{2 \omega 0}$ it was not clear from just the bulk sample measurements if the lower-than-expected measured two-photon polarization was due to the long electron diffusion paths to the cathode surface or to an unexpectedly low value of $P_{2 \omega 0}$, in contradiction to ref. [4.14]. The photoelectron polarization was thus measured using samples with thicknesses significantly less than the electron diffusion length, $L$, with $d \ll l_{s p}$ and $\alpha d \ll 1 .$. GaAs samples, with active thickness of $0.18 \mu \mathrm{m}$ or $0.32 \mu \mathrm{m}$, were analyzed using the same settings of the 
micro-Mott polarimeter as the bulk sample. For the thinnest GaAs sample, the polarizations of both oneand two-photon absorption were only slightly different, $\sim 43 \%$ vs. $40 \%$, respectively, as seen in Table 4.1 . Equations (4.16) and (4.17) detail how polarization depends on GaAs thickness, $L$, and $l_{s p}$. For GaAs with the experimental temperatures and dopants used, the Bir-Aronov-Pikus (BAP) spin-relaxation mechanism is expected to be the dominant depolarization process [4.30]. The BAP process involves the exchange interaction between electrons and holes, and as such will not be constant between the bulk and surface regions, where band bending and the depletion zone will vary the amount of holes. Because of this variation, $l_{s p}$, which is generally taken as a bulk property, is treated as an average over photoemission depth in this work. In addition to the BAP process, additional depolarization mechanisms occur at the GaAs surface, where bulk symmetry is broken and the electron can scatter in the CsF layer [4.31]. Equations (4.16) and (4.17) do not account for depolarization due to surface effects. However, the ratio of one- and two-photon polarization developed in Eq. (4.21) is still valid, as the relative amount of depolarization in both cases would be constant. While theoretically one-photon absorption should give $50 \%$ polarization, the previously mentioned effects reduce the polarization, even with thin active layers. The measured $\sim 43 \%$ polarization at $778 \mathrm{~nm}$ is typical of thin unstrained GaAs [4.32]. The convergence of the two-photon polarization to that of the one-photon polarization with decreasing sample thickness indicates $P_{2 \omega 0}$ close to $50 \%$, as proposed by Bhat et. al [4.14].As such, two-photon absorption is incapable of producing an electron beam with polarization greater than $50 \%$.

Table 4.1: Photoemitted electron polarization taken for one- and two-photon absorption for three different samples of GaAs.

Photoelectron Polarization \%

\begin{tabular}{|c|c|c|} 
Active Thickness & One-photon (778 nm) & Two-photon (1560 nm) \\
$0.18 \mu \mathrm{m}$ & $42.6 \pm 1.0$ & $40.3 \pm 1.0$ \\
$0.32 \mu \mathrm{m}$ & $44.0 \pm 1.1$ & $36.0 \pm 0.9$ \\
Bulk Material & $33.4 \pm 0.8$ & $16.8 \pm 0.4$ \\
\hline
\end{tabular}


An estimate of the spin relaxation length can be made by solving Eq. (4.21) for $l_{s p}$, while letting $\frac{P_{2 \omega 0}}{P_{\omega 0}}=1$, as supported by the experimental evidence, and using the electron polarizations obtained from bulk GaAs for both one- and two-photon absorption, $33.4 \%$ and $16.8 \%$, respectively. With these assumptions, the calculation yields $l_{s p}=2 \cdot 8 \cdot 10^{-5} \mathrm{~cm}$, which is an order of magnitude smaller than reported values. This disparity could be due to the influence of electron scattering in the CsF layer, differences in dopant species, and the strong dependence of $l_{s p}$ on $L$, all of which factors can strongly affect $\alpha, L$, and $l_{s p}$. Perhaps more importantly, the term $\frac{\alpha l_{s p}+1}{\alpha L+1} \approx \frac{1}{2}$ is in close agreement with previous measurements [4.33]. For reference, Table 4.2 lists the values of GaAs material properties determined in this experiment, together with the values of quantities that were used for calculations.

Table 4.2: GaAs material properties as related to one- and two- photon polarized emission.

\begin{tabular}{|c|c|c|}
\hline Quantity & Value & Reference \\
\hline $\begin{array}{l}\text { One photon absorption coefficient } \\
\alpha\end{array}$ & $1.1 \cdot 10^{4} \mathrm{~cm}^{-1}$ & [4.29] \\
\hline Diffusion length $\quad L$ & $1.5 \cdot 10^{-4} \mathrm{~cm}$ & [4.28] \\
\hline $\begin{array}{c}\text { Two-photon absorption coefficient } \\
\beta\end{array}$ & $1.6 \pm 0.5 \mathrm{~cm} / \mathrm{GW}$ & this work \\
\hline $\begin{array}{l}\text { Spin relaxation length } \\
\qquad l_{s p}\end{array}$ & $0.3 \cdot 10^{-4} \mathrm{~cm}$ & this work \\
\hline
\end{tabular}




\subsection{Two-Photon Polarization Conclusions}

Two-photon photoemission was used to generate electron beams from unstrained GaAs photocathodes of varying thickness: $625 \mu \mathrm{m}, 0.32 \mu \mathrm{m}$ and $0.18 \mu \mathrm{m}$. For each photocathode, the degree of spin polarization of the photoemitted beam was less than $50 \%$, contradicting a simple prediction based on quantum mechanical selection rules. In hindsight, a full examination of the quantum selection rules indicates that the transition depicted in Figure 1c is not be allowed, as two photons of like circular polarization must excite electron transitions with a change in azimuthal quantum number $\Delta \ell=2$, which precludes a ${ }^{2} \mathrm{p}_{3 / 2}$ to ${ }^{2} \mathrm{~s}_{1 / 2}$ transition at the $\Gamma$ point.

Polarization via two-photon absorption from the thickest sample was approximately half that obtained via one photon absorption. For the thin samples, polarizations via two and one photon absorption were comparable ( $40 \%$ to $43 \%$, respectively), which is evidence that the maximum possible polarization from two-photon absorption is $\sim 50 \%$, as predicted by Bhat et al. [4.14]. In addition, the two-photon absorption coefficient, $\beta$, was measured to be $1.6 \pm 0.5 \mathrm{~cm} / \mathrm{GW}$, with the spin relaxation length, $l_{s p}$, measured to be $2.810^{-5} \mathrm{~cm}$.

Two-photon excitation of electrons to the conduction band of GaAs, with photon energy equal to one half the band-gap, is not a promising method means to generate highly spin polarized electron beams, as it produces only $40 \%$ polarization, along with very low $\mathrm{QE}\left(\sim 1 \times 10^{-7} \%\right)$. It is possible that other photon energies could be used to excite electrons to forbidden states [4.14] with short lifetimes shorter than the spin decay lifetime. Further work should be done to investigate these forbidden transitions in GaAs, as they may hold the key to creating an inexpensive, highly-polarized electron source. 


\section{K2 CsSb: A High Average Current Photocathode}

While GaAs is currently the only photocathode used to generate spin polarized electron beams, it may not be best source for all electron accelerators. For example, light sources and energy recovery linacs require bright electron beams, often at high average current, and need not be polarized. The photoguns that provide these bright beams must exhibit long operational lifetime to accommodate a demanding User community. The accelerator community can choose between two photogun choices: DC high voltage guns and RF guns. Today's DC high voltage guns primarily use GaAs photocathodes to produce $\mathrm{CW}$ beam, but at modest energy $(<500 \mathrm{kV})$, whereas RF guns use metal, CsTe, or alkaliantimonide photocathodes to produce $\mathrm{MeV}$ beam, but with a low duty factor. Many gun groups, including the CIS, are working diligently to address the limitations of these two approaches.

There are two popular photocathode choices for generating high average current electron beams at accelerators using relatively inexpensive RF-pulsed green-laser light: GaAs and $\mathrm{K}_{2} \mathrm{CsSb}$. The GaAs photocathode can exhibit very high quantum efficiency $(\mathrm{QE})$ and can produce a beam with small thermal emittance [5.1]; however, it requires a very high vacuum in order to ensure that the photocathode surface stays clean on an atomic scale. During operation, GaAs is prone to rapid QE degradation that can result from many situations, such as poor vacuum, high voltage discharges within the gun, and low level field emission. The $\mathrm{K}_{2} \mathrm{CsSb}$ photocathode can reliably exhibit high QE, but whereas GaAs can be purchased from numerous reliable vendors, $\mathrm{K}_{2} \mathrm{CsSb}$ is a compound grown by the User in situ near the gun, by successive application of elemental species on a suitable substrate. Consistent results depend on adherence to proper growth procedures, which have been arrived at empirically across several different groups [5.2][5.3][5.4]. The $\mathrm{K}_{2} \mathrm{CsSb}$ photocathode has a slightly larger thermal emittance as compared to GaAs [5.5], which would affect the maximum brightness of its electron beam, but it is considered to be a 
prompt emitter because of its positive-electron affinity (PEA) nature, producing shorter electron bunches than GaAs. One of the biggest advantages over a GaAs photocathode is the $\mathrm{K}_{2} \mathrm{CsSb}$ photocathode's ability to survive under markedly harsher vacuum conditions compared to GaAs [5.2], which may even allow for its extended use in the relatively poor vacuum of an RF gun.

The basis for these introductory comments stems from reports of accelerator operation at a handful of locations [5.6],[5.7],[5.8],[5.9],[5.10]. The JLab free electron laser (FEL) uses GaAs inside a DC high voltage photogun biased at $360 \mathrm{kV}$, and it routinely operates with $5 \mathrm{~mA}$ average current [5.10], although occasional high voltage discharges necessitate time consuming photocathode reactivation or replacement. In contrast to the relatively low current at JLab, the Boeing FEL used $\mathrm{K}_{2} \mathrm{CsSb}$ inside a normal conducting RF gun and produced a maximum average current of $32 \mathrm{~mA} \mathrm{[5.2].} \mathrm{The} \mathrm{photocathode} \mathrm{provided} \mathrm{high} \mathrm{QE}$ and was very robust, surviving inside a vacuum chamber known to have a leak to the water cooling jacket of the photogun. QE decreased during operation, but it could be restored to $100 \%$ of its original value with the application of more cesium. The Cornell University photogun group recently set impressive new milestones using both types of photocathodes by demonstrating sustained delivery of $20 \mathrm{~mA}$ average current over 8 hours from $\mathrm{K}_{2} \mathrm{CsSb}$ with no observed QE decay [5.11] and $52 \mathrm{~mA}$ over several hours using GaAs with a 100 C charge lifetime [5.12].

This chapter will focus on the performance of $\mathrm{K}_{2} \mathrm{CsSb}$ photocathodes inside a DC high voltage photogun, which had previously been used to characterize the performance of GaAs photocathodes [5.13]. Under nominally identical conditions (gun and beamline vacuum, drive laser wavelength and laser spot size, etc.), the performance of the two photocathodes was both qualitatively and quantitatively assessed.

Several key observations will be discussed in the following chapter. Firstly, the $\mathrm{K}_{2} \mathrm{CsSb}$ photocathode used in the CIS testing was manufactured at one location, and transported many miles to JLab using a modest vacuum transport vessel, without significant QE decay. Ion-bombardment, which is a major source of QE decay for GaAs photocathodes, did not significantly impact the charge lifetime of the $\mathrm{K}_{2} \mathrm{CsSb}$ photocathodes, at least under UHV conditions. Rather, the charge lifetime of $\mathrm{K}_{2} \mathrm{CsSb}$ 
CHAPTER 5: $\mathrm{K}_{2} \mathrm{CsSb}$ : A HIGH AVERAGE CURRENT PHOTOCATHODE

photocathodes was strongly dependent on laser heating. Laser heating induced chemical changes in the $\mathrm{K}_{2} \mathrm{CsSb}$ photocathode that could affect charge lifetime for better or worse. Along with chemical changes that led to QE variations, there were morphological changes in the surface of the photocathodes. The geometric emittance of the photocathode, before and after sustained use, was comparable to reported values.

\subsection{Background Information}

\subsubsection{Emittance Measurement Theory}

Geometric beam emittance describes the momentum distribution of the beam at a given point along the beam path and is an important parameter in the design and operation of an electron accelerator, especially with electron light sources where the beam emittance is inversely proportional to photon brilliance. With a photoemission electron gun, the emittance scale is set by the thermal emittance of the photocathode and plays an important role in the choice of photocathode material. The emittance, of a beam can be described by plotting particle momentum versus position, for directions transverse to the overall beam motion. The distribution forms an ellipse and area of this ellipse gives the beam emittance [5.15]. The emittance of the electron beam at JLab was measured using method wire-scanning technique, aka solenoid scan, [5.16], which involves determining the beam diameter at a point along the beamline for at least three different settings of upstream focusing magnets.

It helps to consider the case of motion of a particle though a beamline which consists of drift spaces and non-dispersive focusing magnets. Summarizing [5.14], the position vector of such a charged particle, in the x-plane, is given by the product of the initial position vector and a transfer matrix;

$$
\mathbf{X}_{\text {final }}=\mathbf{R} \mathbf{X}_{\text {initial }}
$$


with

$$
\mathbf{R}=\left(\begin{array}{ll}
R_{11} & R_{12} \\
R_{21} & R_{22}
\end{array}\right), \text { and } \mathbf{X}=\left(\begin{array}{c}
x \\
x^{\prime}
\end{array}\right)
$$

Similar equations to Eq. (5.1) and (5.2) also hold in the y-plane. For this 2 x 2 matrix representation to describe the motion of the electron adequately, the equations of motion must be decoupled in the $\mathrm{x}$ and $\mathrm{y}$ planes. A more complete representation must be used in the cases of coupled motion [5.15].

A particle beam is just a collection of particles described by the ellipse,

$$
\mathbf{X}^{\mathrm{T}} \sigma^{-1} \mathbf{X}=\mathbf{1}
$$

with the beam matrix, $\sigma$, defined by

$$
\sigma=\left(\begin{array}{ll}
\sigma_{11} & \sigma_{12} \\
\sigma_{21} & \sigma_{22}
\end{array}\right)
$$

The beam matrix can be propagated through the beamline using typical transfer matrix notation as

$$
\sigma_{\text {final }}=\mathbf{R} \sigma_{\text {initial }} \mathbf{R}^{\mathbf{T}}
$$

It follows that the sigma matrix at the end of a given system of magnets and drifts can be expanded in terms of the initial sigma matrix to give

$$
\sigma_{11, \mathrm{f}}=R_{11}^{2} \sigma_{11, \mathrm{i}}+2 R_{11} R_{12} \sigma_{12, \mathrm{i}}+R_{12}^{2} \sigma_{22, \mathrm{i}} .
$$


With the beam radius given by $\sqrt{\sigma_{11}}$, Eq. (5.6) shows that a measurement of the beam size at the exit of a beamline for three different magnet settings is sufficient to determine $\sigma_{\mathrm{i}}$, if the transfer matrix, $\mathbf{R}$, is known. The area of the beam ellipse is the emittance, $\boldsymbol{\epsilon}$, and is then given by

$$
\epsilon=\pi \sqrt{\operatorname{det} \sigma}=\pi \sqrt{\sigma_{11} \sigma_{22}-\sigma_{12}^{2}}
$$

While three measurements is strictly sufficient to measure the emittance, taking more measurements can reduce errors brought about by determining the beam width. For $n$ measurements, Eq. (5.6) can be expressed as

$$
\mathbf{A}=\mathbf{M B}
$$

with

$$
\mathbf{A}=\left(\begin{array}{c}
\sigma_{11,1} \\
\vdots \\
\sigma_{11, \mathrm{n}}
\end{array}\right), \text { and } \mathbf{B}=\left(\begin{array}{c}
\sigma_{11, \mathrm{i}} \\
\sigma_{12, \mathrm{i}} \\
\sigma_{22, \mathrm{i}}
\end{array}\right)
$$

and

$$
\mathbf{M}=\left(\begin{array}{ccc}
R_{11,1}^{2} & 2 R_{11,1} R_{12,1} & R_{12,1}^{2} \\
\vdots & \vdots & \vdots \\
R_{11, n}^{2} & 2 R_{11, n} R_{12, n} & R_{12, n}^{2}
\end{array}\right) .
$$

The least squares solution to find the initial sigma is then

$$
\mathbf{B}=\left(\mathbf{M}^{\mathrm{T}} \mathbf{V}^{-\mathbf{1}} \mathbf{M}\right)^{-\mathbf{1}} \mathbf{M}^{\mathrm{T}} \mathbf{V}^{-\mathbf{1}} \mathbf{A}
$$

in which the errors in the square of the beam radius are given by the diagonal matrix 


$$
\mathbf{V}=\left(\begin{array}{ccc}
\sigma_{1}^{2} & \cdots & 0 \\
\vdots & \ddots & \vdots \\
0 & \cdots & \sigma_{n}^{2}
\end{array}\right)
$$

The errors in the parameters can be calculated from the covariance matrix

$$
\mathbf{c}=\left(\mathbf{M}^{\mathrm{T}} \mathbf{V}^{-\mathbf{1}} \mathbf{M}\right)^{-\mathbf{1}}
$$

The beamline used for this testing at JLab consisted of a solenoidal focusing magnet followed by a drift space, then another solenoid, and then a final drift space. Two solenoids are used in the emittance measurement, as one would couple the $\mathrm{x}$ and $\mathrm{y}$ motion together. Using a second solenoid, identical to the first but for an opposite electric current, the transfer matrix will be decoupled. The complete transfer matrix is then

$$
\mathbf{R}=\mathbf{D}_{\mathbf{2}} \mathbf{S}_{-} \mathbf{D}_{\mathbf{1}} \mathbf{S}_{+}
$$

with $\mathbf{S}_{+}$the first solenoid, $\mathbf{D}_{1}$ the first drift of length $L_{1}, \mathbf{S}$. the second solenoid, and $\mathbf{D}_{\mathbf{2}}$ the second of length $L_{2}$. Using typical transfer matrices for solenoids and drift spaces [5.16] and multiplying through, the elements of Eq. (5.14) can be given in either the $\mathrm{x}$ or $\mathrm{y}$ plane as

$$
\begin{aligned}
& R_{11}=C^{2}-S^{2}-L_{1} L_{2} K^{2} S^{2}-\left(L_{1}+2 L_{2}\right) K S C \\
& R_{12}=\left(L_{1}+L_{2}\right) C^{2}-L_{2} S^{2}+\frac{2 S C}{K}-L_{1} L_{2} K S C \\
& R_{21}=L_{1} K^{2} S^{2}-2 K S C \\
& R_{22}=C^{2}-S^{2}-L_{1} K S C .
\end{aligned}
$$

The new constants seen in Eq. (5.15) come from the physical properties of the beamline. $C=\cos \left(K L_{s}\right)$, $S=\sin \left(K L_{s}\right)$, where $L_{s}$ is the effective length of the solenoid. $K=B(0) / 2 B \rho_{0}$, with $B(0)$ the peak field inside the solenoid and $B \rho_{0}$ the magnetic rigidity of the beam. 
CHAPTER 5: $\mathrm{K}_{2} \mathrm{CsSb}$ : A HIGH AVERAGE CURRENT PHOTOCATHODE

The procedure to measure the emittance of a particle beam has a few steps. First, the beam is scanned at the end of the final drift space, using n different solenoid settings. Next, the beam width is determined. Finally, the matrix M, Eq. (5.10) is filled in using the n magnetic field settings, and Eq. (5.11) is solved for the initial beam matrix, $\sigma_{i}$.

\subsubsection{GaAs Photocathode QE Decay Mechanisms}

As discussed in Chapter 2, photoemission is obtained from GaAs only after application of $\sim$ one monolayer of $\mathrm{Cs}$ and an oxidant, which for the CIS is fluorine as supplied by $\mathrm{NF}_{3}$, in order to create a NEA surface. This NEA surface is the reason that a GaAs cathode can achieve a very high $\mathrm{QE}$, even at its band-gap energy. In general, the $\mathrm{QE}$ of any photocathode is not constant with respect to time. There are two major kinds of QE decay mechanisms: those related to static vacuum conditions, and those triggered during beam generation. The first kind is the QE decay that might be encountered under static vacuum conditions and is primarily related to chemical poisoning of the activated photocathode surface. This poisoning occurs when residual gases, such as water, oxygen, or carbon dioxide, even at very small pressures, react with chemicals used to create the NEA condition [5.18]. Because this decay is independent of any beam production, it is known as the dark lifetime of a photocathode, and is generally measured in units of time, with many modern laboratories able to prepare GaAs cathodes with dark lifetimes up to several thousand hours.

These very high dark lifetimes indicate that gases released by electron-stimulated desorption are primarily the cause of any chemical poisoning of the photocathode, and not residual gases. Electrons that trigger ESD can come from either field emission of the gun cathode electrode structures, or from electron beam loss along the beamline, with the former source able to be largely eliminated [5.19]. In general, if the beam if properly managed by limiting the active area of the photocathode, by properly baking vacuum chambers and beamlines, and by properly steering the beam, ESD is of minimal concern. Because chemical poisoning is dependent on residual gas interactions with the photocathode surface, it affects the 
QE uniformly over the entire photocathode wafer, unlike the dominant QE decay mechanism, which is ion bombardment, and as such the two processes can be differentiated.

Ion bombardment, or ion backbombardment, is the second major vacuum issue that leads to the loss of $\mathrm{QE}$ in GaAs. Ion bombardment is dependent on residual gas interactions with the electron beam, and as such, its effects are only seen during beam operation. Ions are produced by the beam and these positive ions are then electrostatically attracted back towards the cathode, as shown in Figure 5.1.

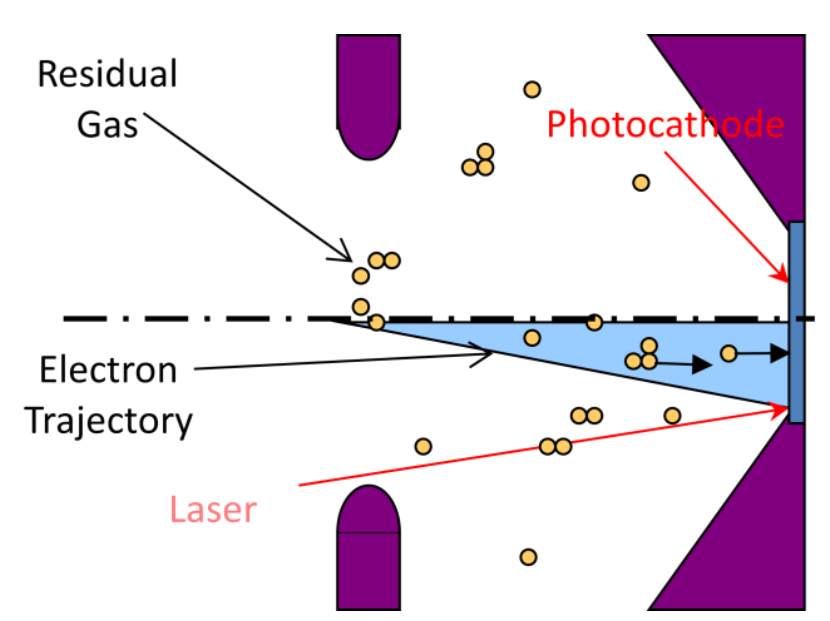

Figure 5.1: Diagram of ion backbombardment of a photocathode. Electrons travel from origination on the photocathode towards the center of the beam line. Ions created impact largely at the electrostatic center of cathode.

Depending on the mass and energy of the positive ion, it can damage the photocathode via implantation in the semiconductor, or by sputtering away the activation layer. Because photocathodes are located in electrode structures that focus and accelerate the electron beam, any ions produced by these electrons will impact the photocathode along a line connecting the beam-point-of-origin and the electrostatic center [5.21]. Evidence of ion bombardment can be seen in Figure 5.2, which shows QE maps of a photocathode used at CEBAF. The series of images shows QE degradation originating from three different photocathode locations. Notice the QE trench that connects each laser site to the electrostatic center. 

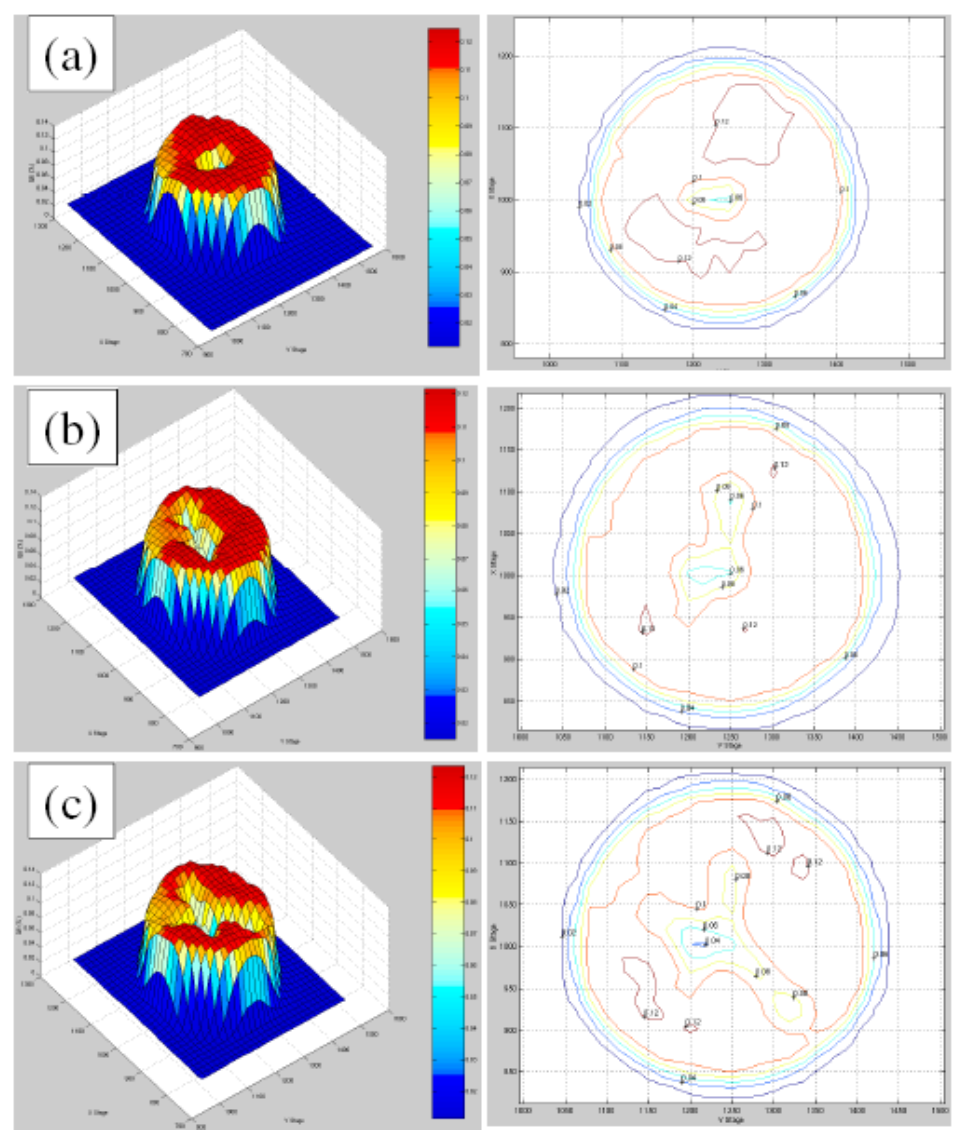

Figure 5.2: $\mathrm{QE}$ map of a GaAs photocathode, showing characteristic $\mathrm{QE}$ decay due to ion bombardment over a period of many weeks. The QE damage is along a line that joins the location of laser spots to the electrostatic center of the photocathode. From Ref [5.19].

The ill-effect of ion bombardment can be minimized in a number of ways. The first way is to improve the vacuum conditions of the gun [5.20], while another way is to use a larger laser spot size of lower intensity (giving the same power) [5.22]. This larger spot size serves to spread out the ion damage over a larger area, thus increasing the amount of beam that can be run. Many more techniques, such as limiting the activated area of the cathode [5.22], running the beam farther from the electrostatic center, and biasing the anode, can be employed to lengthen the operational lifetime of a GaAs, but eventually, the QE will always decay to unusable levels. Some amount of lost QE can be regained by reheating and re-activating the photocathode; however, even this process has its limits. At some point, the GaAs photocathode must be replaced, a time consuming process that must be avoided for as long as possible in order to ensure a 
CHAPTER 5: $\mathrm{K}_{2} \mathrm{CsSb}$ : A HIGH AVERAGE CURRENT PHOTOCATHODE

high percentage of beam uptime for a demanding User base at any accelerator facility. The aforementioned problems with GaAs QE decay are some of the reasons that there is presently much interest in the use $\mathrm{K}_{2} \mathrm{CsSb}$ as a high current photocathode.

\section{2 $\quad \mathrm{K}_{2} \mathrm{CsSb}$ Photocathode Experimental Methodology}

\subsubsection{Photocathode Preparation}

Two $\mathrm{K}_{2} \mathrm{CsSb}$ photocathodes were manufactured at Brookhaven National Laboratory (BNL) inside an ultra-high vacuum deposition chamber (base pressure $\sim 2 \times 10^{-11}$ Torr) by the sequential deposition of high-purity $\mathrm{Sb}, \mathrm{K}$, and $\mathrm{Cs}$ onto a heated substrate, referred to as a "puck". Photocathode \#1 was fabricated April 1, 2011 and photocathode \#2 was fabricated on November 18, 2011. Each photocathode was delivered to JLab a few days of fabrication, in the manner described below. The source of antimony was high-purity $\mathrm{Sb}$ pellets, resistively heated in a tungsten crucible, while the $\mathrm{K}$ and Cs sources consisted of SAES alkali dispenser strips for photocathode \#1, similar to the Cs source used in the micro-Mott for GaAs activation [3.4], and Alvatec source vials for photocathode \#2 [5.18]. Figure 5.3 shows the entire BNL deposition chamber. 

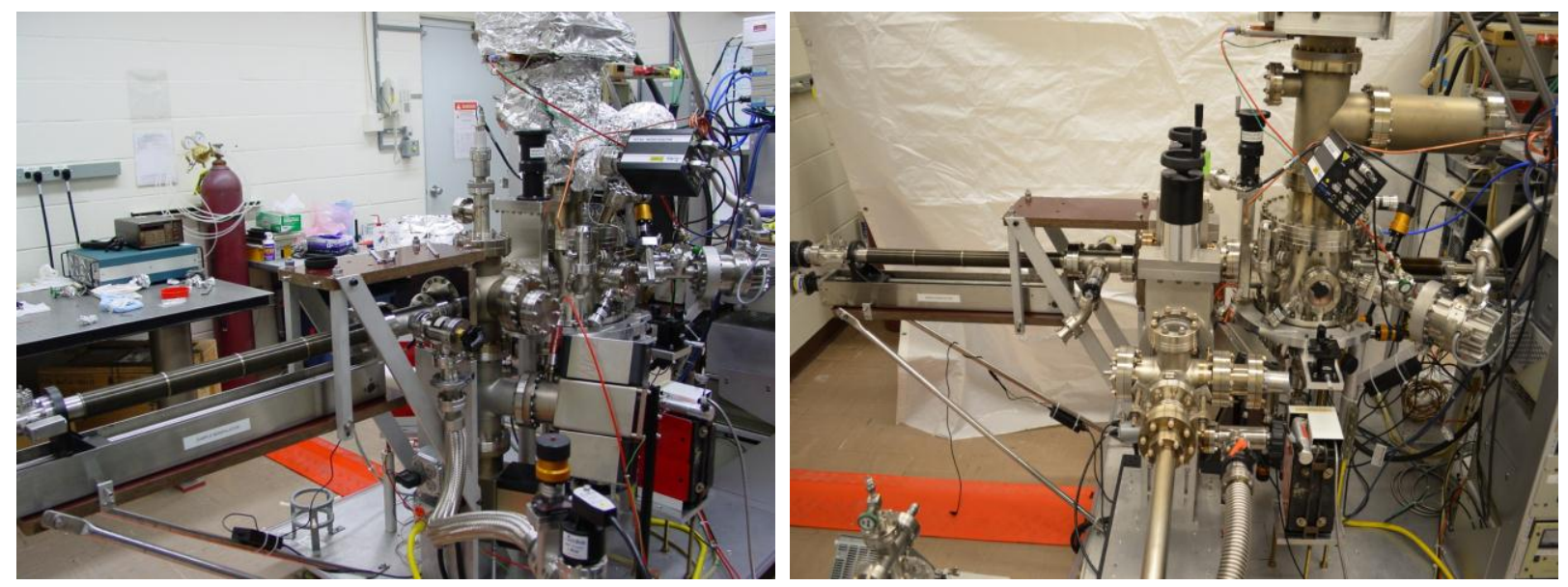

Figure 5.3: Two views of the BNL deposition system used for fabrication of $\mathrm{K}_{2} \mathrm{CsSb}$ photocathodes.

The puck on which the $\mathrm{K}_{2} \mathrm{CsSb}$ photocathode was grown was similar in shape and size to pucks used at CEBAF for securing GaAs photocathodes [5.13]. It differed from the standard stainless steel JLab puck because it was made of aluminum with only a thin layer of stainless steel explosion bonded to the top, which served as the coating substrate. Thin layers of titanium and copper were between the stainless steel substrate surface and the bulk of the aluminum puck, and were needed for the explosion bonding process; explosion bonding was the only known way to attach the stainless steel substrate to the aluminum puck. The substrate must be heated during the photocathode fabrication; the heater inside the BNL deposition chamber had only modest heating capability, and as such, aluminum was chosen for the puck body because of its relatively small mass combined with good thermal heat conduction. Stainless steel was chosen as the photocathode substrate as previous measurements at BNL indicated it provided high QE while illuminated with $532 \mathrm{~nm}$ light [5.24]. The stainless steel surface was polished using diamond paste with $9 \mu \mathrm{m}$ grit, cleaned with a citric-acid soap in an ultrasonic bath, rinsed in de-ionized water and then vacuum baked to $200^{\circ} \mathrm{C}$ inside a load lock system before insertion into the deposition chamber. This cleaning process is the standard process that the CIS group uses before inserting new equipment into exiting vacuum chambers. Figure 5.4 shows a photograph of the puck resting in a fork 
holder, composed of $\mathrm{BeCu}$, which was used to sequentially translate the puck over the three deposition sources as needed.

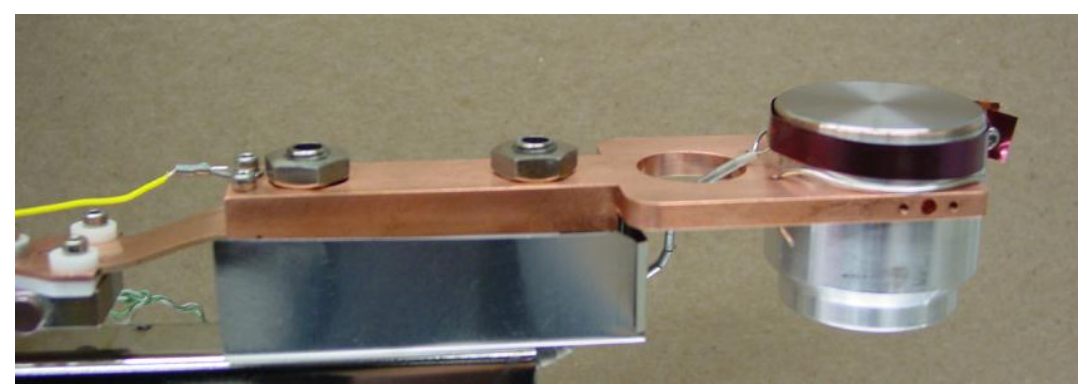

Figure 5.4: The transfer arm of the $\mathrm{BNL} \mathrm{K}_{2} \mathrm{CsSb}$ deposition system holding the stainless steel and aluminum puck. The puck can be biased for QE measurements, and heated and cooled.

No attempt was made to limit the photocathode active area - the entire stainless steel substrate was coated with photocathode chemicals. A typical GaAs puck at CEBAF uses a mask to protect all but the center $5 \mathrm{~mm}$ of the semiconductor wafer from the activation chemicals, $\mathrm{Cs}$ and $\mathrm{NF}_{3}$ for the CIS, which limits the production of photocurrent to only the center of the photocathode. This masking is shown to increase the charge lifetime of GaAs by limiting the amount of electrons that can be emitted from areas with poor beam transport. The BNL deposition system was not equipped for this center masking, and as such, no mask was used.

The fork and puck could be heated to $150{ }^{\circ} \mathrm{C}$ via resistive heating and cooled to $-80{ }^{\circ} \mathrm{C}$ via tubes capable of handling liquid nitrogen. The assembly could be biased to $5 \mathrm{kV}$, which allowed QE measurements to be made during and after the depositions. To control the deposition rate of the $\mathrm{Sb}$ and $\mathrm{K}$ sources, a calibrated crystal microbalance was used. The following deposition procedure was used:

1. The puck was heated to $100{ }^{\circ} \mathrm{C}$ and $\sim 14 \mathrm{~nm}$ of $\mathrm{Sb}$ was applied to the substrate at a rate of $\sim 0.5 \mathrm{~nm} / \mathrm{s}$.

2. The puck temperature was raised to $140{ }^{\circ} \mathrm{C}$ and $30 \mathrm{~nm}$ of $\mathrm{K}$ was applied at a rate of $\sim 0.5$ $\mathrm{nm} / \mathrm{s}$. 
CHAPTER 5: $\mathrm{K}_{2} \mathrm{CsSb}$ : A HIGH AVERAGE CURRENT PHOTOCATHODE

3. The puck was cooled to $135^{\circ} \mathrm{C}$, biased at $-20 \mathrm{~V}$, and then illuminated with $\sim 0.5 \mathrm{~mW}$ of 532 nm light.

4. Cs was applied while monitoring the extracted photocurrent, until photocurrent ceased to increase.

5. The puck was quickly cooled to room temperature.

The QE spectrum of both photocathodes, taken just after reaching room temperature, is shown in Figure 5.5. The QE at $532 \mathrm{~nm}$ was $0.8 \%$ and $2.8 \%$, for photocathode \#1 and \#2, respectively. The QE of photocathode \#1 was lower than expected, possibly as a result of the $\mathrm{K}$ dispenser becoming depleted during fabrication. In an effort to increase the QE of the first photocathode, approximately $4 \mathrm{~nm}$ of additional Sb was applied before adding more Cs until photocurrent was at a maximum. The low QE may have resulted from lack of proper stoichiometry due to the excess of $\mathrm{Sb}$ and $\mathrm{Cs}$. This excess may have led to the formation of either $\mathrm{Cs}_{3} \mathrm{Sb}$ or $\mathrm{K}_{2} \mathrm{CsSb}$, or a mixed state of both. The $\mathrm{QE}$ spectrum for photocathode \#2 is a more typical result for deposition on a stainless steel substrate. In the UV, the peak QE of both photocathodes was over 20\%. Further information concerning the growth methods and QE spectral measurements can be found in reference [5.24]. 
CHAPTER 5: $\mathrm{K}_{2} \mathrm{CsSb}$ : A HIGH AVERAGE CURRENT PHOTOCATHODE

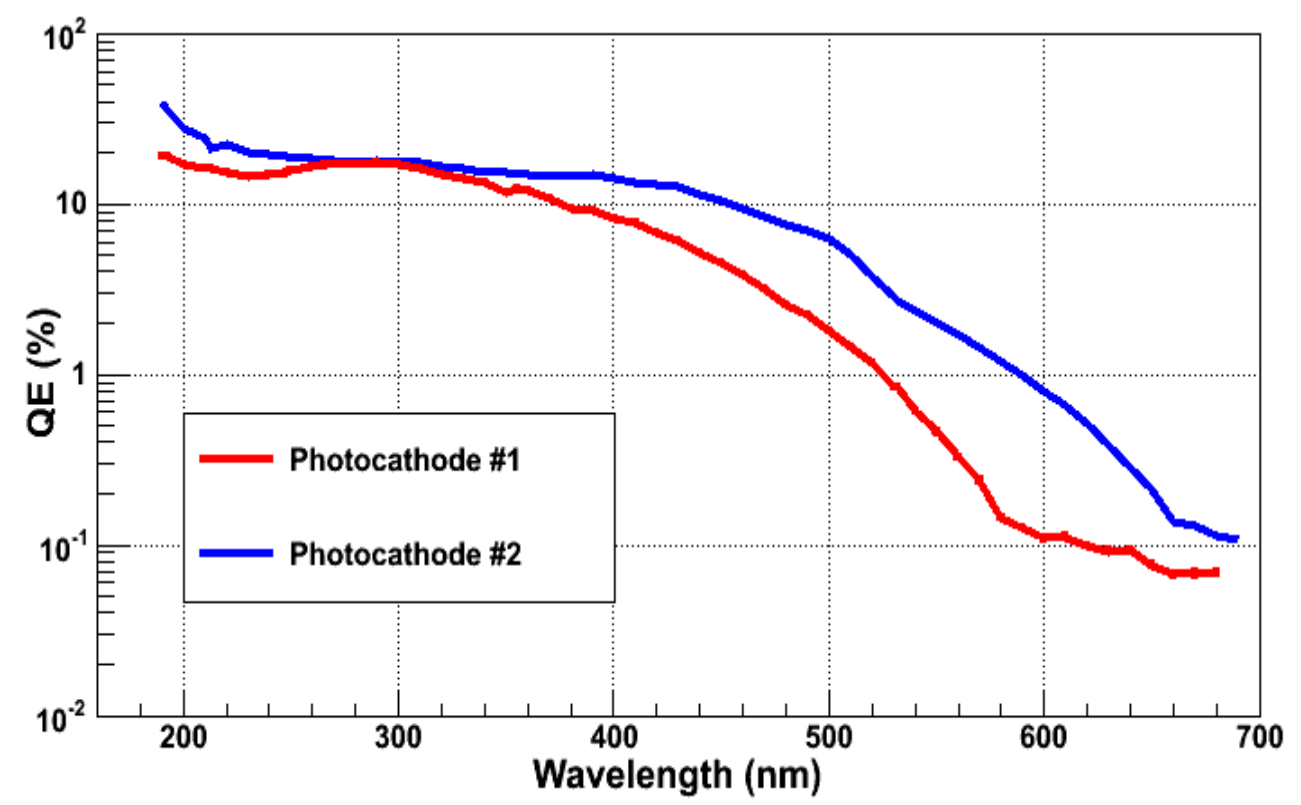

Figure 5.5: Spectral response of the $\mathrm{K}_{2} \mathrm{CsSb}$ photocathodes created at $\mathrm{BNL}$ just after deposition.

\subsubsection{Photocathode Transfer}

After fabrication, the deposition chamber was prepped for the photocathode transfer. A vacuum "suitcase", shown in Figure 5.6, composed of a rotating/translating UHV sample manipulator with "cradle" attached at the end used to hold the puck, a 6-way CF vacuum cross, and an all-metal gate valve opposite the manipulator, was attached to the deposition chamber by means of vacuum Tee, with an ion pump attached. The vacuum Tee piece mated to the BNL deposition chamber via another all-metal gate valve. The suitcase is depicted in Figure 5.7 while attached to the BNL deposition chamber. After connection, the suitcase system was baked to $200{ }^{\circ} \mathrm{C}$. While most vacuum component bakes for the CIS go to $250{ }^{\circ} \mathrm{C}$, the temperature was limited due to the thermal constraints of the manipulator. After the suitcase bake, the puck was pulled from the deposition vacuum chamber and moved into the suitcase. The suitcase pressure was $\sim 10^{-11}$ Torr achieved using of a small ion pump $\left(20 \mathrm{~L} / \mathrm{s} \mathrm{N}_{2}\right)$ in combination with a NEG pump $\left(600 \mathrm{~L} / \mathrm{s} \mathrm{H}_{2}\right)$. Once the puck was installed inside the suitcase, the gate valve was closed and 
CHAPTER 5: $\mathrm{K}_{2} \mathrm{CsSb}$ : A HIGH AVERAGE CURRENT PHOTOCATHODE

the puck and cradle were then pressed against the back face of the gate valve in order to prevent the puck from falling out during transit. Figure 5.8 shows the puck in the cradle inside of the suitcase during the handoff from the deposition chamber. During transport, the manipulator was moved into the suitcase chamber, moving the puck out of the plane of the page of Figure 5.8. In order to prevent the puck from falling out of the cradle during transport, the manipulator was fully inserted into the suitcase chamber. The side of the puck visible in Figure 5.8 was pushed against the valve, leaving the photocathode surface undisturbed. The suitcase was then packed into a van for transport to JLab, with the trip duration from BNL to Jefferson Lab (450 miles) taking approximately 10 hours. The ion pump in the suitcase was powered during the entire trip, which, along with the NEG pump, kept the pressure inside the suitcase at $\sim 10^{-11}$ Torr, with occasional intermittent pressures spikes to $10^{-9}$ Torr at when the van encountered bumps in the road.

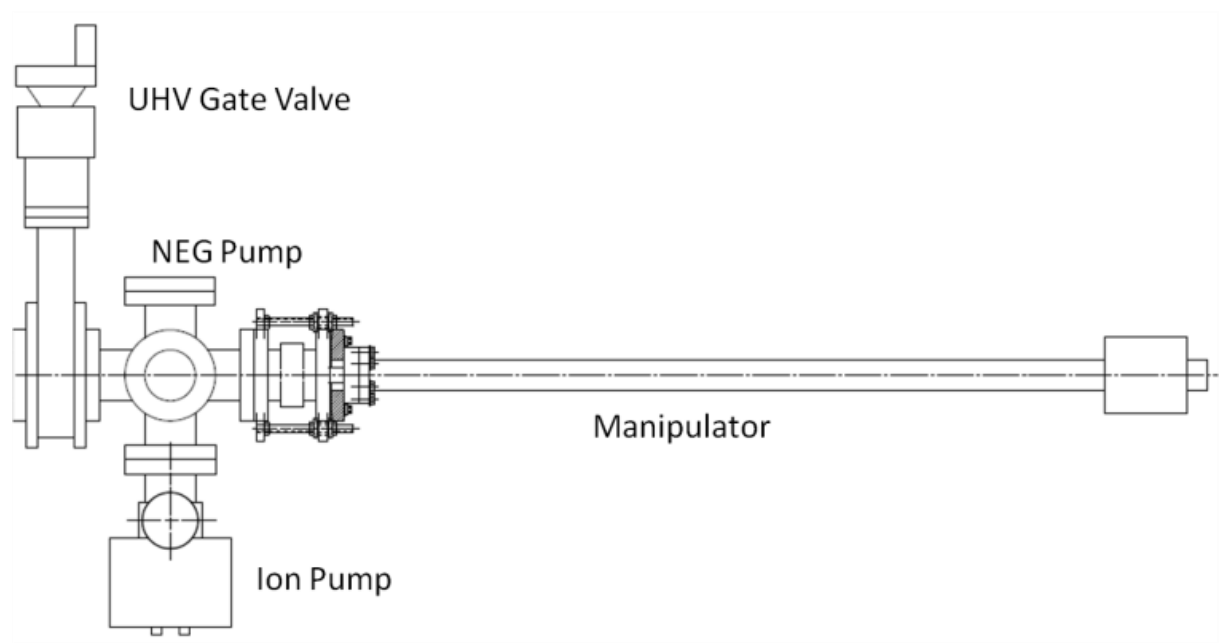

Figure 5.6: Schematic of the vacuum suitcase composed of a magnetic sample manipulator, 6-way cross with 4.5" Conflat flanges, gate valve and vacuum pumps. 


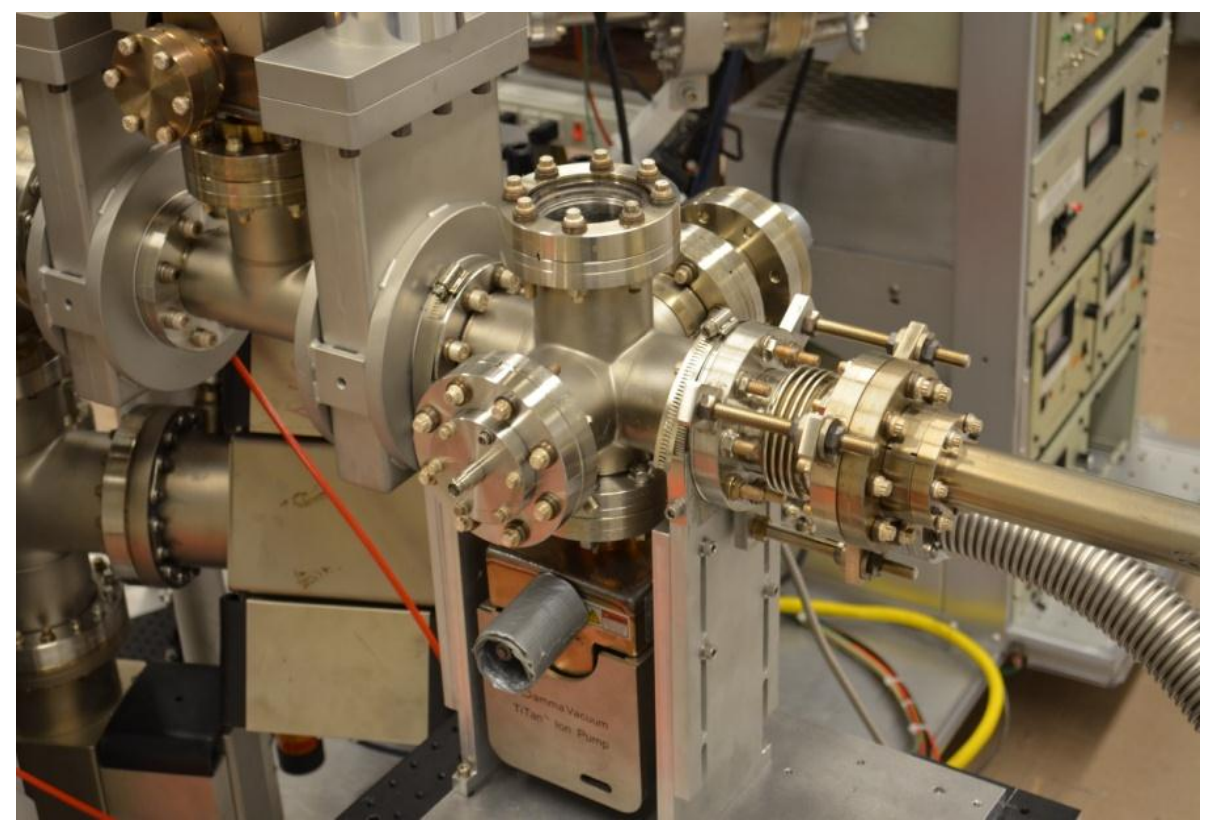

Figure 5.7: The JLab vacuum suitcase attached to the BNL deposition chamber.

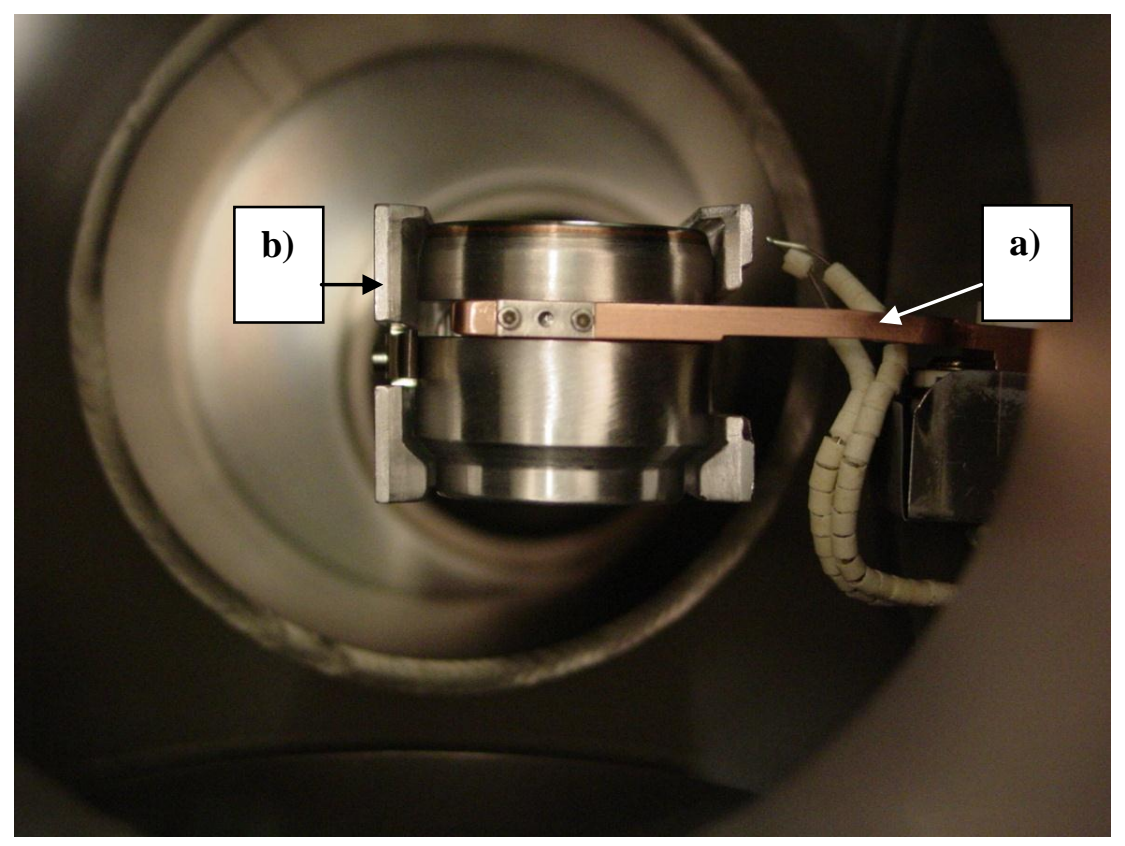

Figure 5.8: Photo of the photocathode puck transfer between the BNL deposition chamber and the JLab suitcase. The $\mathrm{BeCu}$ fork (a) passes the puck into the cradle (b) of the manipulator in the suitcase. The manipulator enables movement in and out of the page, as well as rotation of the puck. 


\subsubsection{Jefferson Lab Photogun Testing Equipment}

Immediately upon arrival at Jefferson Lab, the vacuum suitcase was attached to a another small vacuum Tee that connected to an all-metal gate valve on the GaAs preparation chamber of JLab's Injector Test Stand (ITS) Facility. A schematic of the entire ITS can be seen in Figure 5.9, with an overhead view of the suitcase, preparation chamber, and HV photogun chamber shown in Figure 5.10. The vacuum Tee, (incorrectly shown as a cross in Figure 5.9), was evacuated and baked for twelve hours at $100^{\circ} \mathrm{C}$ in order to achieve vacuum $\sim 1 \times 10^{-10}$ Torr. These modest bakeout conditions were chosen due to worries about the thermal stability of the $\mathrm{K}_{2} \mathrm{CsSb}$ photocathode. The QE of the photocathode was measured occasionally during the bakeout procedure, and beyond these bake parameters, the QE of the photocathode was observed to decrease slightly, although vacuum did not appreciably degrade. Following bakeout, the gate valves at the preparation chamber and suitcase were opened, and the $\mathrm{K}_{2} \mathrm{CsSb}$ photocathode was quickly transferred first to the preparation chamber and then into the photogun high voltage chamber. Note that the preparation chamber, which normally serves to activate GaAs photocathodes, was not used for this experiment, except as a transfer chamber. $\mathrm{K}_{2} \mathrm{CsSb}$ photocathode was quickly transferred through the unused preparation chamber in order to limit any possible contamination of the surface. The load locked photogun is described more fully in reference [5.25]. 
CHAPTER 5: $\mathrm{K}_{2} \mathrm{CsSb}$ : A HIGH AVERAGE CURRENT PHOTOCATHODE
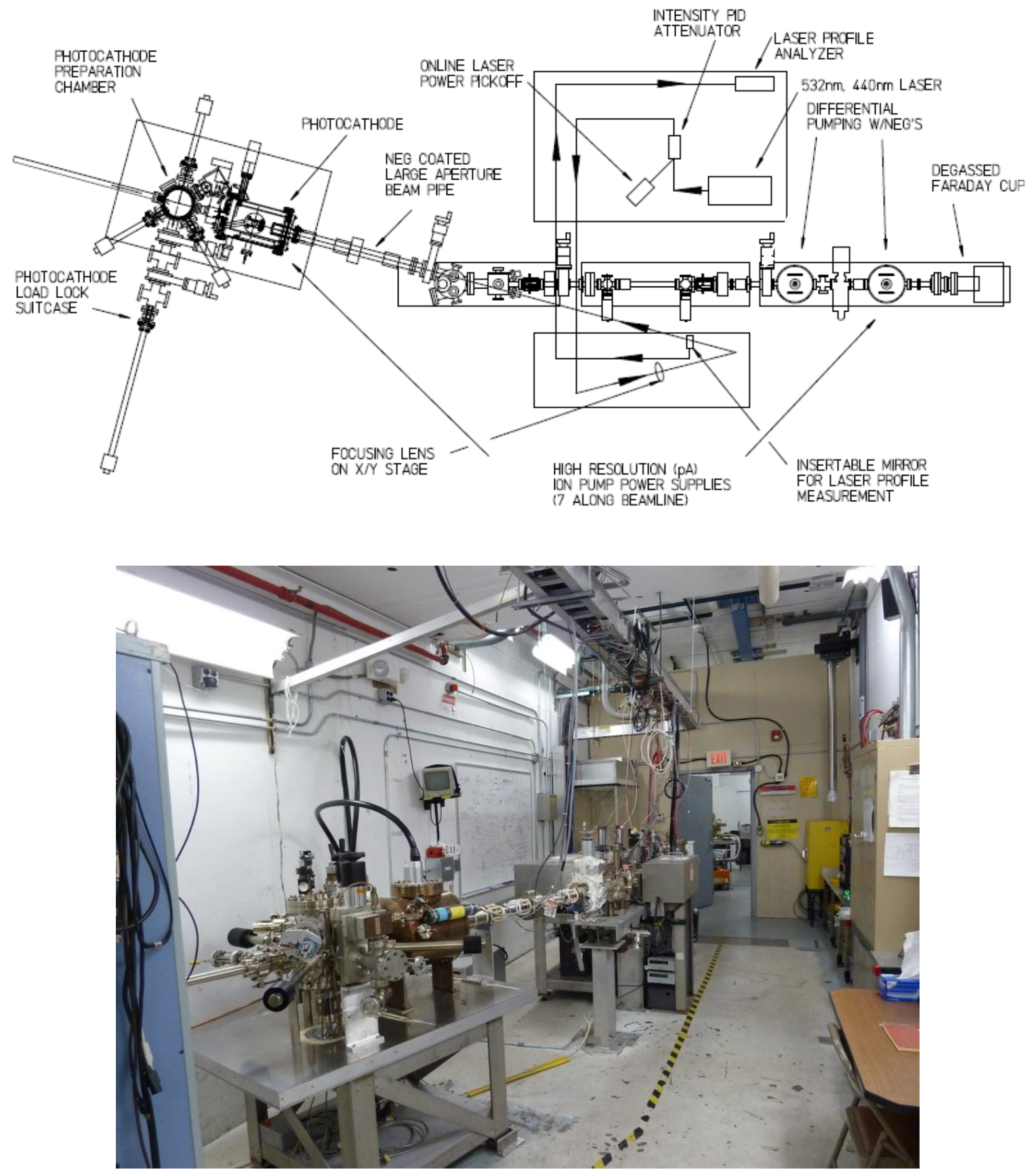

Figure 5.9: Schematic(upper) and photo (lower) of the photogun and beamline at JLAB's Injector Test Stand Facility. 


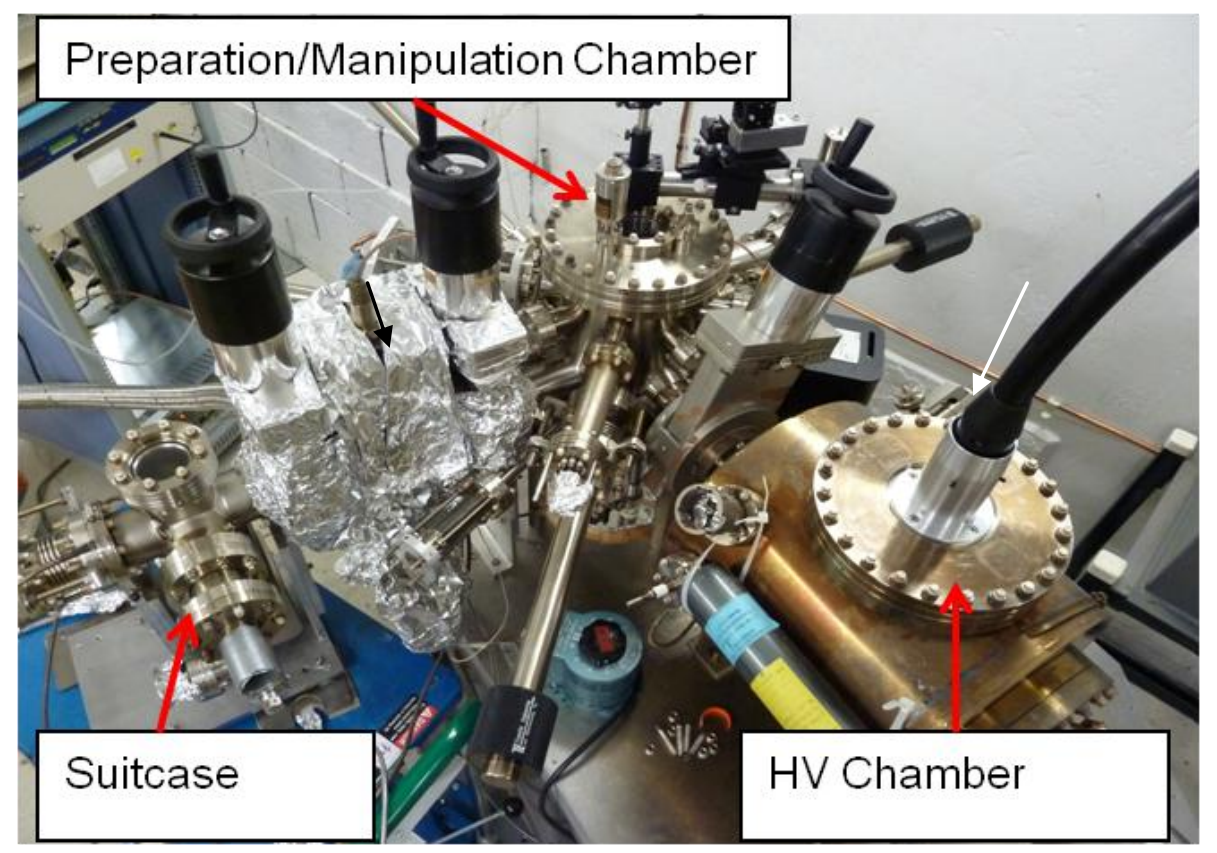

Figure 5.10: Photograph of the photogun and preparation chamber of the ITS, with the transfer suitcase attached.

A cut-away schematic of the HV photogun chamber of the ITS is shown in Figure 5.11. The photocathode was inserted into a large-grain niobium electrode with $25^{\circ}$ focusing angle [5.26]. Photoemitted electrons were accelerated to a kinetic energy up to $200 \mathrm{keV}$ over a $6.3 \mathrm{~cm}$ cathode/anode gap. The ring anode was electrically isolated and attached to a sensitive current monitor that could detect both field emission and photoemission that did not pass through the anode. The cathode electrode hangs from an alumina insulator $(11 \mathrm{~cm}$ long) that extends into the vacuum chamber. Ten non-evaporable getter (NEG) pump modules [5.27] surround the cathode/anode gap. The NEGs, in combination with an ion pump, provide pressure $2 \times 10^{-12}$ Torr (uncorrected, $\mathrm{N} 2$ equivalent). 


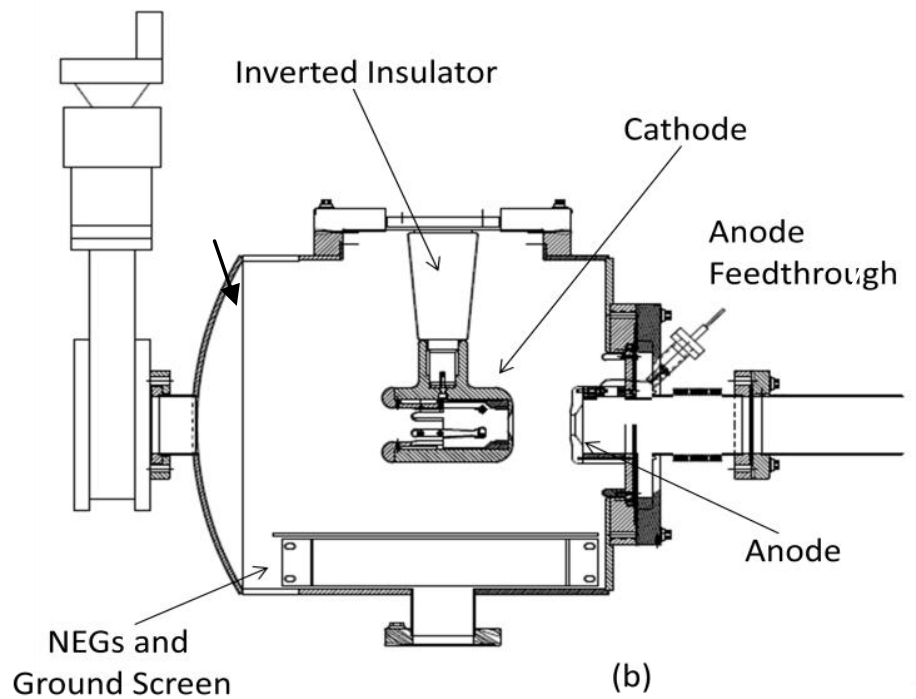

Figure 5.11: Schematic of the DC high voltage photogun at JLab, with inverted ceramic insulator.

Photoelectrons passed through the anode and were delivered to a water-cooled Faraday cup beam dump $\sim 5 \mathrm{~m}$ away. Solenoid magnets focused the beam and weak steering magnets kept the beam centered in the beampipe. The Faraday cup was baked at $450^{\circ} \mathrm{C}$ for 24 hours in order to reduce outgassing at high currents. Two differential pumping chambers with NEG pumps vacuum-isolated the beam dump from the photogun. Ion pumps, which had sensitive current monitoring [3.26], pumped inert gasses which were not pumped by the NEGs. The ion pumps also provided beamloss monitoring along the beamline, as the current monitoring was sensitive to increases in vacuum pressure brought about by electrons striking beam pipe. In addition to using the ion pump current supplies, the quality of the beam tune was monitored with the use of $\mathrm{x}$-ray detectors located along the beam line and gun chamber.

A $15^{\circ}$ bend and vacuum window located in a $\mathrm{Y}$ vacuum chamber provided a means to introduce drive laser light onto the photocathode at normal incidence, without requiring mirrors inside the vacuum chamber. Light reflected from the photocathode exited the vacuum chamber via the laser vacuum window within $\sim 0.1^{\circ}$ of the incident laser beam, which indicated a proper alignment of the photocathode, cathode, anode, laser, and beam line. 
Two laser systems were used: a frequency-doubled Nd:YVO 4 laser (Coherent Verdi-10) provided up to $4.5 \mathrm{~W}$ at the photocathode at $532 \mathrm{~nm}$, and an inexpensive multimode diode laser provided up to 0.2 $\mathrm{W}$ at $440 \mathrm{~nm}$. Both lasers were operated in DC mode. Laser power at the photocathode was adjusted via a computer controlled attenuator consisting of a fixed linear polarizer, a rotatable birefringent $\lambda / 2$ wave plate, and another fixed linear polarizer, similar to the optical attenuator used for the separate micro-Mott polarimeter previously discussed. The attenuator was calibrated by measuring the laser power passed through the system for any given rotation of the $\lambda / 2$ wave plate. A focusing lens was mounted to $x / y$ stepper motor translation stages near the laser vacuum window, which allowed the laser spot to be positioned anywhere on the photocathode. The $532 \mathrm{~nm}$ laser had excellent mode quality and could be tightly focused to $500 \mu \mathrm{m}$ Gaussian full-width at half-maximum (FWHM) using a $2 \mathrm{~m}$ focal length lens. A larger laser spot $(850 \mu \mathrm{m})$ was obtained using a lens with $1.5 \mathrm{~m}$ focal length. The spatial mode quality of the $440 \mathrm{~nm}$ diode was not nearly as good as the $532 \mathrm{~nm}$ laser, and required an anamorphic prism pair to create a relatively uniform spatial profile, which then focused to nearly Gaussian profile with tightest beam size $1 \mathrm{~mm}$ FWHM. A commercial CCD camera and scanning razor-blade apparatus, which was located at an equivalent distance to the photocathode along a different path, were used to measure both the laser profile and spot size, as well as to verify that the laser beam was circular, with approximately Gaussian profile. The optical elements (mirrors, wave plates, polarizing cubes) were all purchased with appropriate coatings for the two used wavelengths.

\subsection{4 $\quad \mathrm{K}_{2} \mathrm{CsSb}$ Testing Procedure}

The experiment was mostly an exercise in measuring photocathode QE, before, during and after a particular "run" in which one of the following parameters were varied: elapsed time, beam current, location of the laser beam on the photocathode, laser beam spot size, laser power, laser wavelength, and photogun voltage. Photocathode QE could be measured "real time" by extracting electron beam while 
CHAPTER 5: $\mathrm{K}_{2} \mathrm{CsSb}$ : A HIGH AVERAGE CURRENT PHOTOCATHODE

monitoring both the delivered photocurrent to the beam dump as well as the incident laser power by means of a calibrated partial reflector. The laser power could also be measured by noting the setting of the calibrated laser attenuator. Before and after a run, the QE of the entire photocathode surface was measured by extracting $\sim 1 \mu \mathrm{A}$ from the grounded photocathode with the anode biased at $\sim 375 \mathrm{~V}$, while scanning the laser across the photocathode by translating the focusing lens on stepper motor stages, in a procedure known as a QE scan, which could take anywhere from one to twenty minutes, depending on the desired spatial resolution of the scan. Lasers were left powered on throughout the duration of the experiment (and shuttered when beam was not extracted) to minimize laser power drift, which was less than $3 \%$ drift over 12 hours. Unless otherwise noted, measurements were made at fixed beam current by means of a software feedback loop that controlled the laser attenuator and thus the amount of laser power that reached the photocathode. The term charge lifetime is defined as the amount of charge that could be extracted from the photocathode before QE fell to 1/e of the initial value.

Beam emittance was determined using the wire scanner technique previously described [5.14], which is also known as the solenoid scan technique. The size of the electron beam was measured using three thin wires, two of which were orthogonal, passing through the beam, for different settings of the last solenoid magnet.

\section{3 $\quad \mathrm{K}_{2} \mathrm{CsSb}$ Beam Lifetime Results}

\subsubsection{Photocathode \#1: Charge Lifetime - Initial 532 nm}

The initial QE scan of photocathode \#1 showed that the QE ranged from 0.5 to $0.8 \%$ across the $12.8 \mathrm{~mm}$ diameter surface (Figure 5.12a). The QE remained constant for days before extracting beam, which suggests a very long dark lifetime under the vacuum conditions in the ITS photogun. The 
CHAPTER 5: $\mathrm{K}_{2} \mathrm{CsSb}$ : A HIGH AVERAGE CURRENT PHOTOCATHODE

photocathode was biased at $100 \mathrm{kV}$, and charge lifetime measurements were performed at different radial locations. The beam current was $1 \mathrm{~mA}$ and the charge extracted for each run ranged from 6 to $30 \mathrm{C}$. Following each run, a QE scan was performed (Figure 5.12b shows the QE several runs along the Y=0 axis) and an exponential fit was applied to the QE decay. Charge lifetime measurements are plotted in Figure 5.13, as a function of radial position of the laser beam relative to the electrostatic center of the photocathode. For ease of viewing, measurements taken at similar radii from the electrostatic center, but along different directions, have been averaged. Also plotted in Figure 5.13 are averaged measurements from a bulk GaAs photocathode [5.13] using the same photogun and drive laser, but with $2 \mathrm{~mA}$ average current. It was both surprising and disappointing that the charge lifetime of the $\mathrm{K}_{2} \mathrm{CsSb}$ photocathode was comparable to, or even worse than, that of $\mathrm{GaAs}$, as from previous work [5.2]. $\mathrm{K}_{2} \mathrm{CsSb}$ was expected to have a higher lifetime. Furthermore, the lifetime dependence on the radial position of the laser beam at the photocathode suggested ion bombardment played a role in QE decay, which was not an effect previously noted [5.2]. As the electron beam travels from the photocathode, residual gases in the vacuum chamber can become ionized. Ion bombardment happens when ions produced by the beam are preferentially focused toward the center of the photocathode in comparison to beam origination location. Typically in GaAs, lifetime can be enhanced by operating with the laser beam displaced from the photocathode center, so that the back bombarded ions do not damage the wafer surface, as can be seen in the linear response of lifetime with distance for GaAs in Figure 5.13. This first test seemed to indicate that ion back bombardment was also of considerable effect in $\mathrm{K}_{2} \mathrm{CsSb}$, as its charge lifetime seemed to have a slight linear relationship with distance from the electrostatic center, although it is hard to draw definite conclusions from the data. 


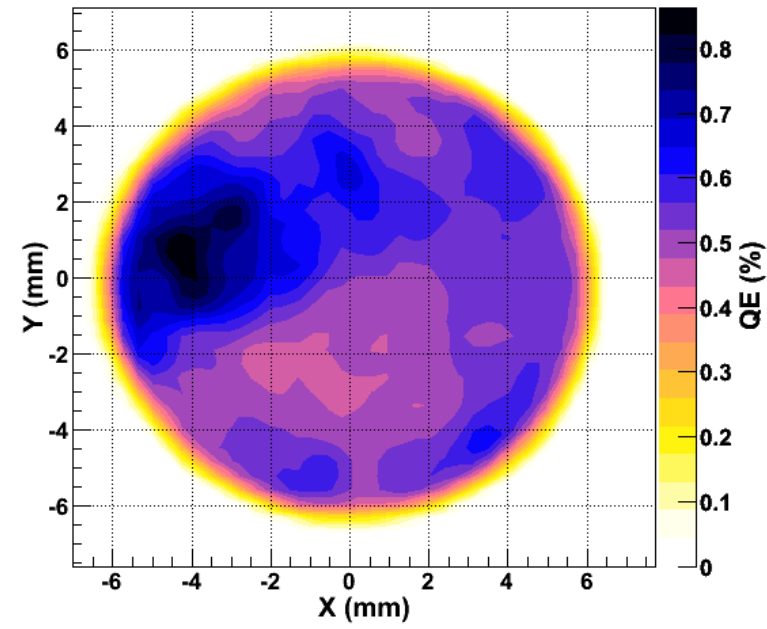

(a)

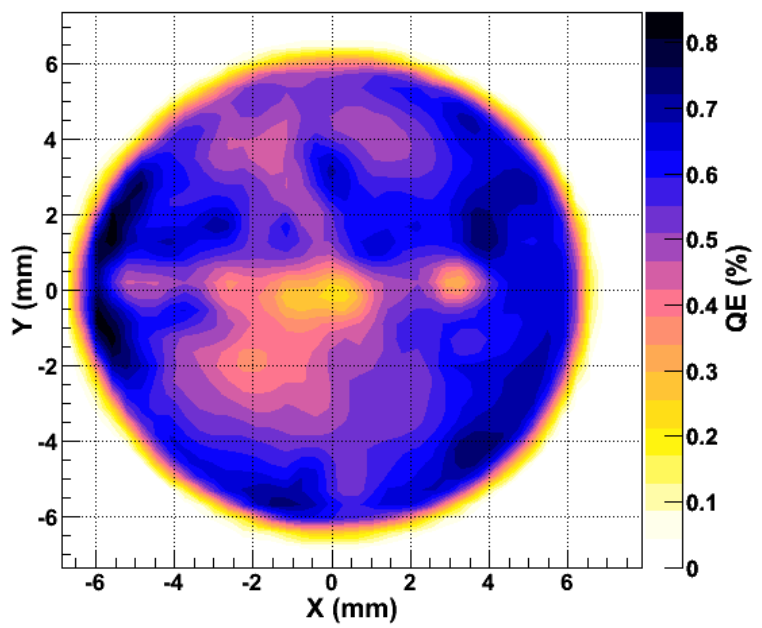

(b)

Figure 5.12: QE maps of $\mathrm{K}_{2} \mathrm{CsSb}$ photocathode \#1 using $532 \mathrm{~nm}$ light when the photocathode exhibited poor lifetime: (a) before running beam and (b) after running beam from many locations, some of which are seen as the low $\mathrm{QE}$ spots along the $\mathrm{Y}=0 \mathrm{~mm}$ axis.

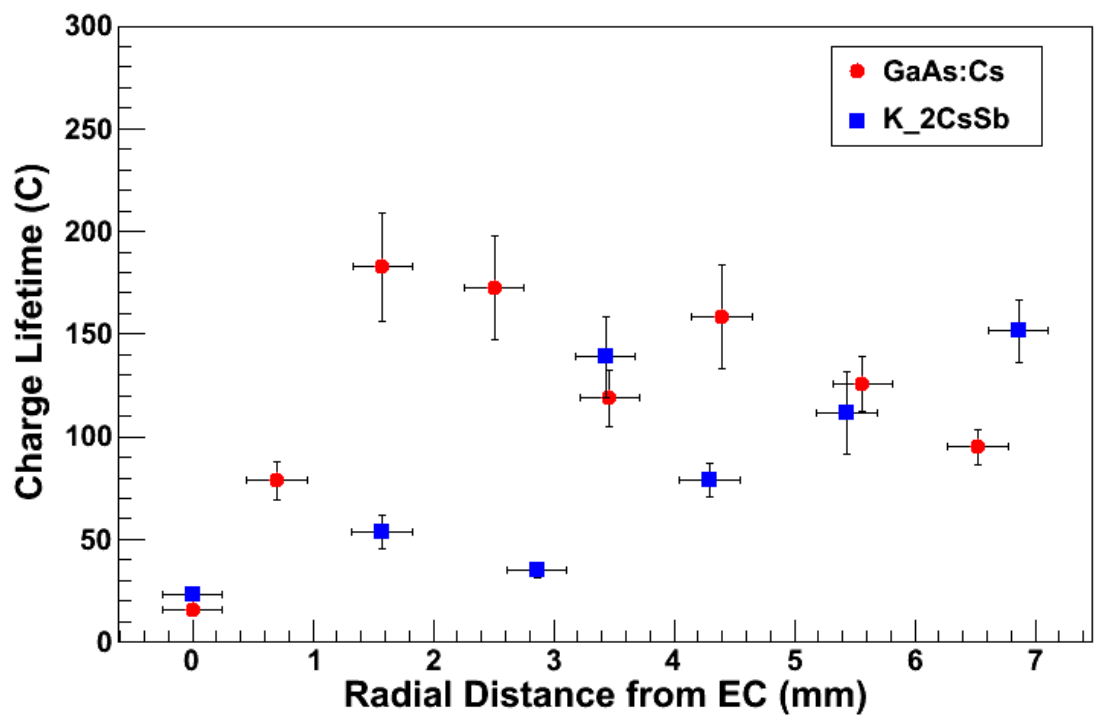

Figure 5.13: Charge lifetime of $\mathrm{K}_{2} \mathrm{CsSb}$ photocathode \#1 ( $\square$ ) and GaAs:Cs (•) versus the radial position of the laser spot relative to the electrostatic center of the cathode. The vertical error bars are related to the quality of the exponential fit to the QE decay curves, and the horizontal error bars reflect uncertainty of the laser position relative the electrostatic center. 
CHAPTER 5: $\mathrm{K}_{2} \mathrm{CsSb}$ : A HIGH AVERAGE CURRENT PHOTOCATHODE

\subsubsection{Photocathode \#1: Charge Lifetime - $440 \mathrm{~nm}$ and Repeated $532 \mathrm{~nm}$}

After ten runs at $532 \mathrm{~nm}$ at different currents and distances from the electrostatic center, all with similarly poor lifetime, runs on the first photocathode delivered from BNL were performed at $440 \mathrm{~nm}$. Light at $440 \mathrm{~nm}$ was chosen for this set of runs, as $440 \mathrm{~nm}$ appeared to be above the work function energy of the first $\mathrm{K}_{2} \mathrm{CsSb}$ photocathode, as indicated in Figure 5.5. In sharp contrast to the previous runs with $532 \mathrm{~nm}$ light, the charge lifetime of $\mathrm{K}_{2} \mathrm{CsSb}$ photocathode \#1 was markedly better at $440 \mathrm{~nm}$. Using gun bias voltages of both 100 and $200 \mathrm{kV}$, initial runs at $1 \mathrm{~mA}$ from the electrostatic center indicated that QE only increased with time. This effect was surprising, as beam delivery from the electrostatic center using a GaAs photocathode would have been very short lived due to ion bombardment (Figure 5.13) [5.13].

While delivering $3 \mathrm{~mA}$ beam at $200 \mathrm{kV}$ using the $440 \mathrm{~nm}$ laser during an unattended run, a brief local power glitch caused three steering magnets to turn off. As a result, beam was misteered into a valve and the flange of a bellows. Because the software current lock was enabled, more laser light was applied to the photocathode in a failed attempt to maintain $3 \mathrm{~mA}$ at the dump. This condition persisted for approximately 1.5 hours, with an increasing amount of total beam delivered to the valve. The vacuum at the photogun degraded to $\sim 10^{-9}$ Torr during this beam delivery. Fortunately, the beamline was not damaged to the point of creating a leak and vacuum recovered quickly once beam was terminated. The entire affected flange (Figure 5.14) heated enough to burn masking tape on the edges of the mu-metal shielding the beamline. The QE was then measured across the entire photocathode at both $440 \mathrm{~nm}$ and $532 \mathrm{~nm}$ wavelengths, with a profound reduction in QE observed at the center of the photocathode (Figure 5.15); surprisingly, the $\mathrm{QE}$ at the edge was nearly unchanged. Such a vacuum event would have eliminated the QE across the entire surface of a GaAs photocathode. Presumably, the QE reduction at the center of the photocathode was a result of ion damage due to prolonged beam extraction under poor vacuum conditions. 


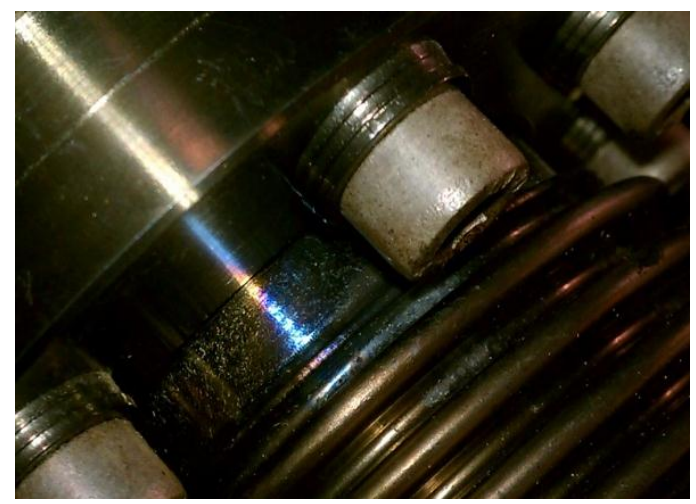

(a)

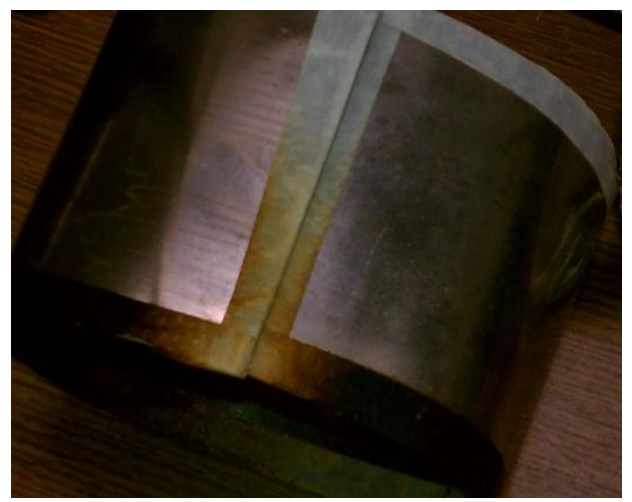

(b)

Figure 5.14: Photos of the beamline flange (a) and its mu-metal shielding (b), showing signs of heating caused by misteered electron beam.

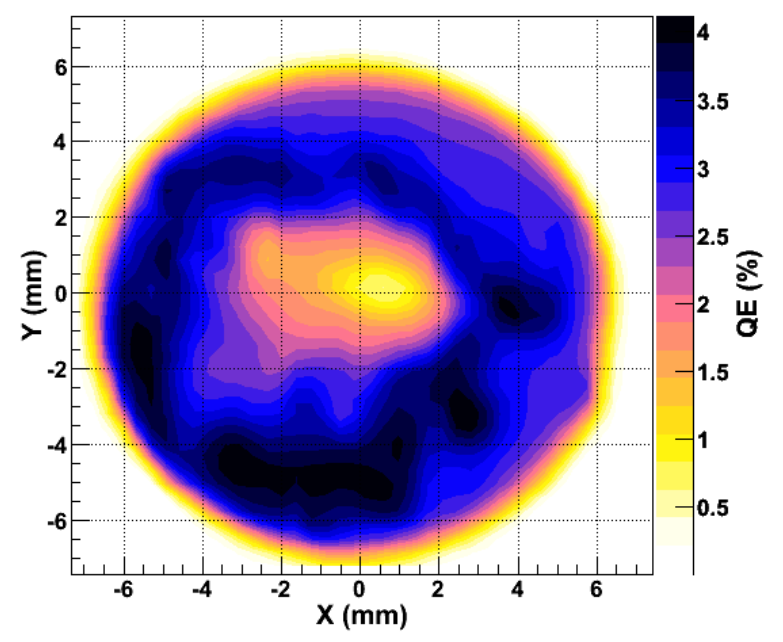

(a)

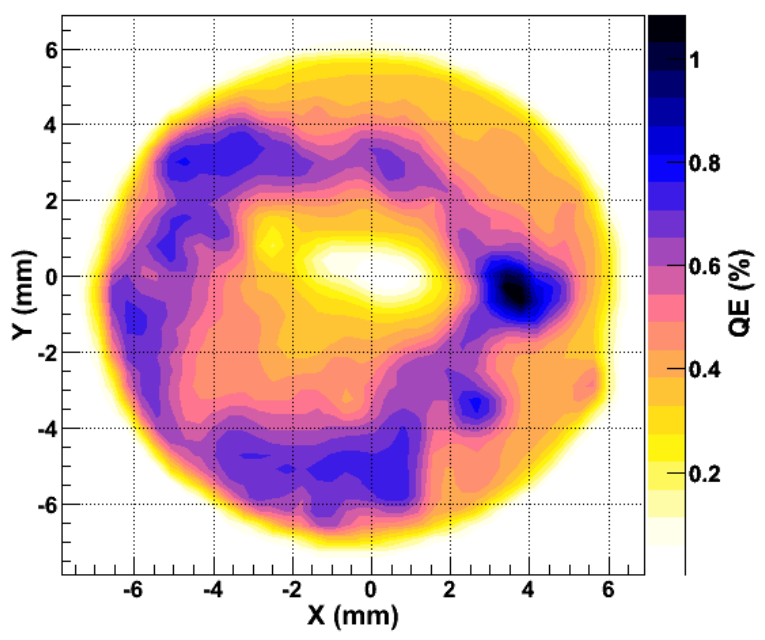

(b)

Figure 5.15: QE scans following the vacuum event during which greater than $1.5 \mathrm{~mA}$ of beam was dumped into a valve for an extended period of time: (a) $440 \mathrm{~nm}$ light and (b) $532 \mathrm{~nm}$ light. Absence of QE in the wafer center was an indication that photocathode material was sputtered away by ions produced by the extracted beam. The high QE spot in (b) at approximate location $\mathrm{x} / \mathrm{y}=4,0 \mathrm{~mm}$ was produced after extracting $3 \mathrm{~mA}$ using $440 \mathrm{~nm}$ light.

Following the beam strike/vacuum event, the charge lifetime of the photocathode at $440 \mathrm{~nm}$ was still very good, as it was before the event. QE increased increase during lengthy runs with currents up to $5 \mathrm{~mA}$, which was the maximum current for the available laser power. Because the center of the photocathode exhibited no QE, beam delivery for these runs was from the edge of the photocathode, 
CHAPTER 5: $\mathrm{K}_{2} \mathrm{CsSb}$ : A HIGH AVERAGE CURRENT PHOTOCATHODE

where efficient transport of the beam was problematic due to an astigmatic imperfection of the cathode/anode electrostatic optic. As a result, a small amount of beam halo intercepted beamline phosphorescent viewers along the beamline, which then could charge up and disturb the beam orbit. Eventually, the viewers would discharge, with the orbit subsequently recovering. This good/bad beam delivery behavior is evident in Figure 5.16a, in which frequent discontinuities in the QE curve are seen. Because QE was calculated by measuring the current observed at the beam dump, and not from the beam that left the photocathode, poor beam orbit, with less than full transport to the dump, caused the observed QE to be artificially low. The sharp QE drops seen in Figure 5.16a therefore correspond to mis-steered beam at the dump, which resulted from the charged viewers affecting the electron beam orbit.

After the surprisingly good lifetime results while extracting beam with $440 \mathrm{~nm}$ light, electron beam was again run at $532 \mathrm{~nm}$. During these second set of beam runs with $532 \mathrm{~nm}$ light, the charge lifetime of the photocathode was greatly improved (Figure 5.16b). The cause of the improvement in lifetime is unknown, but it is speculated that running the beam in poor vacuum conditions resulted in a scrubbing of the surface of the photocathode, favorably altering its chemistry to $\mathrm{K}_{2} \mathrm{CsSb}$ from the suspected $\mathrm{Cs}_{3} \mathrm{Sb}$ stoichiometry. Higher and higher currents were extracted from the photocathode using $532 \mathrm{~nm}$ light with a $500 \mu \mathrm{m}$ laser spot $(\mathrm{FWHM})$. The QE increased over time at currents up to $\sim 5 \mathrm{~mA}$. The QE was relatively stable with $10 \mathrm{~mA}$ beam current, while for current $>16 \mathrm{~mA}$ the $\mathrm{QE}$ decreased (Figure 5.17a). A larger laser spot $(850 \mu \mathrm{m}$ FWHM) was used to extract beam and the QE was observed to decay more slowly than with the smaller spot at beam currents between 15 and $20 \mathrm{~mA}$, corresponding to 26 and $35 \mathrm{~mA} / \mathrm{mm}^{2}$, respectively (Figure $5.17 \mathrm{~b}$ ). 


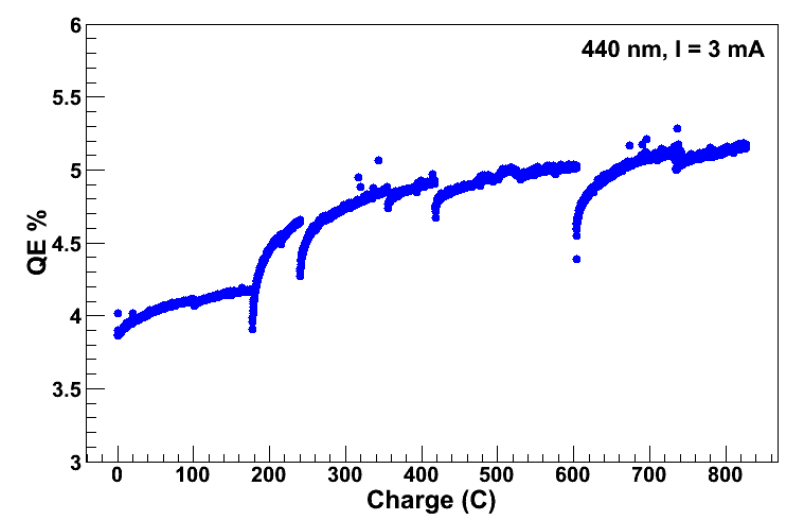

(a)

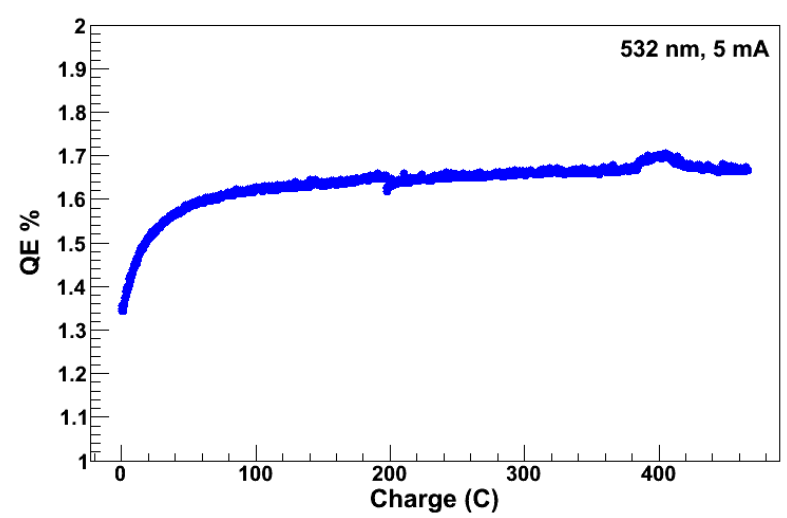

(b)

Figure 5.16: QE evolution during beam delivery from $\mathrm{K}_{2} \mathrm{CsSb}$ photocathode \#1: (a) using $440 \mathrm{~nm}$ light and (b) 532 $\mathrm{nm}$ light. Note $\mathrm{QE}$ increasing with time. The discontinuities are due to static charge build up on viewers and eventual discharges that move the beam at the Faraday cup.

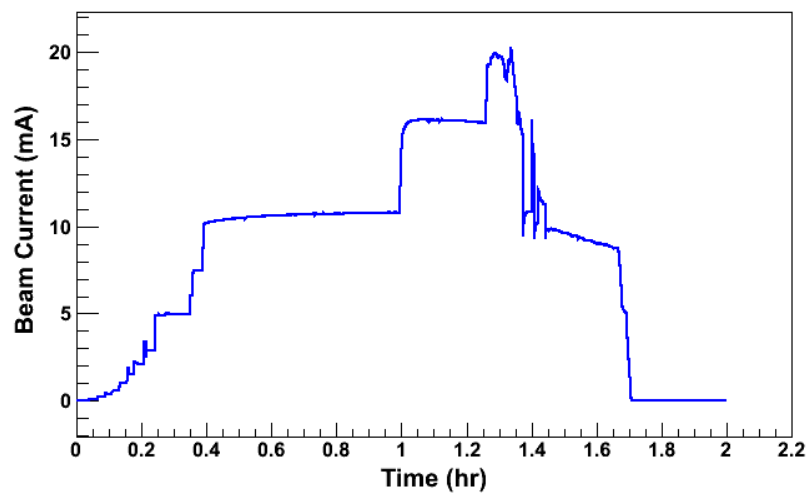

(a)

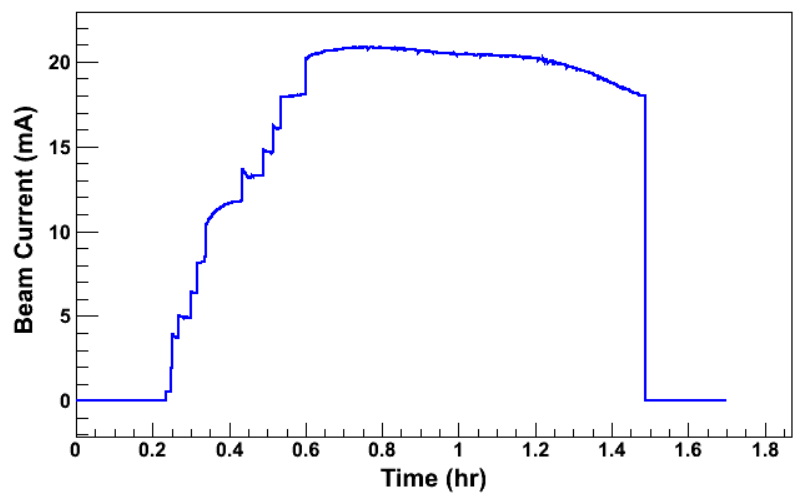

(b)

Figure 5.17: Beam current versus time using $\mathrm{K}_{2} \mathrm{CsSb}$ photocathode \#1 and $532 \mathrm{~nm}$ light with laser spot diameter: (a) $500 \mu \mathrm{m}$ FWHM and, (b) $850 \mu \mathrm{m}$ FWHM 
CHAPTER 5: $\mathrm{K}_{2} \mathrm{CsSb}$ : A HIGH AVERAGE CURRENT PHOTOCATHODE

\subsubsection{Photocathode \#1: Laser Heating}

In order to explain the $\mathrm{QE}$ evolution of $\mathrm{K}_{2} \mathrm{CsSb}$ photocathodes - in particular, the observed $\mathrm{QE}$ increase at low beam currents, the QE decrease at high current, and the charge lifetime enhancement while using a larger laser spot - it was hypothesized that some sort of heating effect brought about by the laser was influencing the QE. At low laser intensities, brought about by either the lower total laser power required at low currents or by using a larger spot size to reduce the intensity on the photocathode, the QE seemed to increase with time. It was thought that low heating brought about by the laser caused chemical changes on the photocathode surface, which possibly brought it closer to a true $\mathrm{K}_{2} \mathrm{CsSb}$ stoichiometry from the $\mathrm{Cs}_{3} \mathrm{Sb}$ form that was thought to have been deposited onto the surface during photocathode growth. At higher laser intensities, the localized heat on the photocathode would have likely been enough to disassociate the Cs from the surface, causing a lower QE over time.

To check the validity of the laser heating hypothesis, the QE of the photocathode was monitored while under the laser, but without extracting beam. By doing so, the influence of the laser, and its possibly heating effects, could be separated from effects due to Joule heating or ion bombardment. To this end, the photocathode was grounded while the drive laser illuminated the photocathode surface for a certain time. The QE of the photocathode was mapped before and after laser illumination duration of either $12 \mathrm{hr}$ or $2 \mathrm{hr}$, and then the two data sets were subtracted to create a QE difference map (Figure 5.18). There was a slight offset in $x / y$ coordinates that resulted in imperfect cancellation. At lower power for both laser wavelengths (200 mW at $440 \mathrm{~nm}$ for $12 \mathrm{hr}$, and $700 \mathrm{~mW}$ at $532 \mathrm{~nm}$ for $2 \mathrm{hr}$ ), the QE was observed to increase following sustained illumination, with the photocathode at ground potential (i.e., there was no extracted electron beam). Because the observed QE increase of the previous charge lifetime runs could be replicated with only the illumination of the laser, it was further thought that a heating effect brought about by the laser was the cause of the QE changes, in agreement with earlier indications. 


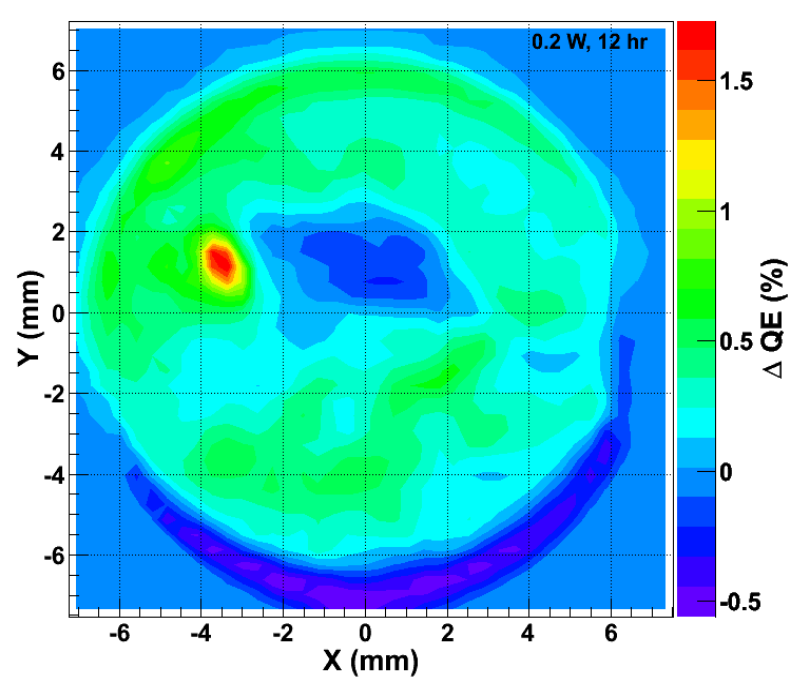

(a)

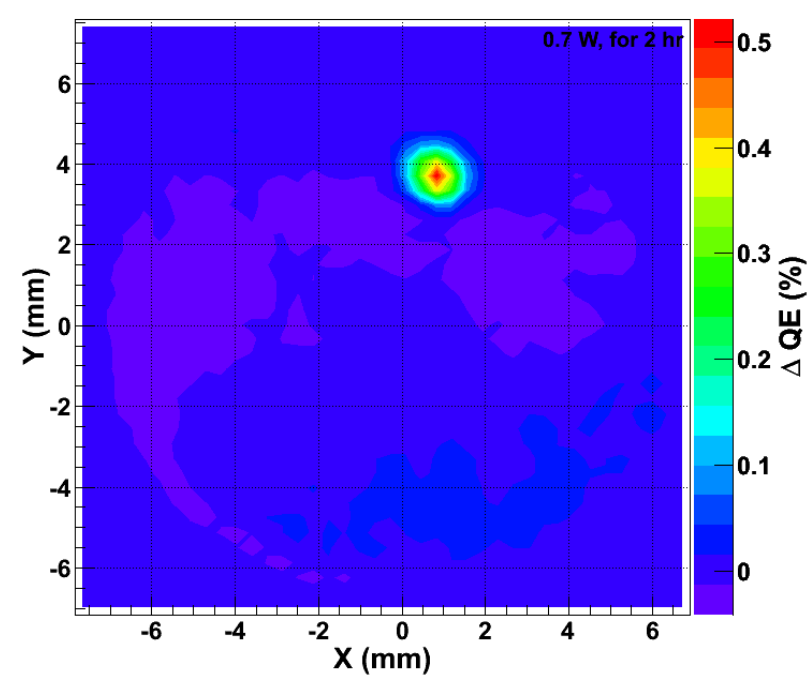

(b)

Figure 5.18: QE difference scans of $\mathrm{K}_{2} \mathrm{CsSb}$ photocathode \#1 using; (a) $440 \mathrm{~nm}$ laser at $200 \mathrm{~mW}$ for $12 \mathrm{hrs}$ and (b) $532 \mathrm{~nm}$ laser at $700 \mathrm{~mW}$ for $2 \mathrm{hrs}$. The photocathode was at ground potential, no beam was extracted while the laser was illuminating the photocathode for the indicated amount of time.

\subsubsection{Photocathode \#2: Ion Bombardment - Biased and Grounded Anode}

Photocathode \#2 was manufactured, without the K deposition problems experienced with the first photocathode, and the QE was satisfactorily high across the surface, with a value of $2.8 \%$ at $532 \mathrm{~nm}$ measured immediately after deposition. Approximately a week and a half after manufacture, the photocathode was transported to JLab, in the same manner as the first photocathode. Upon arrival at Jefferson Lab, QE had decreased by nearly a factor of three as referenced to the initial QE. It is not completely understood why the dark lifetime of this photocathode was so markedly different from photocathode \#1, although it is a possibility that the extended time between growth and transport while being stored in the original deposition system caused chemical changes on the surface of the photocathode. In addition, qualitatively worse road conditions during the second transport could have negatively impacted the suitcase vacuum, leading to the observed reduction in QE. 
After the testing of photocathode \#1 seemed to indicate that laser heating may by the dominant cause over ion-bombardment of the $\mathrm{QE}$ evolution of a $\mathrm{K}_{2} \mathrm{CsSb}$ photocathode, the main goal assigned to the beam tests with photocathode \#2 was to rigorously correlate observed QE evolution with some physical mechanism, in particular, to distinguish between ion-bombardment and any chemical changes that might occur due to laser heating, i.e. disassociation or evaporation of the bialkali material. To address this, $10 \mathrm{~mA}$ of beam was delivered to the dump using $532 \mathrm{~nm}$ laser light positioned at the electrostatic center of the photocathode, while iteratively biasing and grounding the anode electrode during $\sim 1$ hour-long intervals. Previous tests with GaAs (shown in Figure 5.19) indicated that photocathode charge lifetime could be enhanced significantly ( 20\% improvement) at the electrostatic center if ions generated by the beam downstream of the anode were repelled away from the cathode by applying a positive voltage to the electrically isolated anode [5.13]. As shown in Figure 5.20, the test with $\mathrm{K}_{2} \mathrm{CsSb}$ photocathode \#2 showed no lifetime dependence on anode voltage, as the QE decay was the same for both biased and grounded anodes. This behavior suggests that alkali-antimonide photocathodes do not suffer greatly as a result of ion-bombardment, at least in an UHV environment. This result is in slight disagreement with the earlier charge lifetime runs on photocathode \#1 (Figure 5.13), which seemed to indicate a small ion bombardment effect due to the influence of distance from electrostatic center on the lifetime. In addition, all $\mathrm{QE}$ at the center of photocathode \#1 was lost following the beam mis-steer and consequent vacuum event. However due to the vastly different vacuum conditions between running the beam into a flange and running the beam during the anode test, these observations are not necessarily contradictory; ion bombardment may hold influence in bad vacuum, and not be a dominant effect in good vacuum ( $10^{-12}$ Torr). Because of the difference in the charge lifetime of photocathode \#1 measured at $532 \mathrm{~nm}$ before and after the vacuum event, the initial tests on photocathode \#1 and the anode tests on photocathode \#2 cannot be strictly compared, which again results in no contradictory results. 
CHAPTER 5: $\mathrm{K}_{2} \mathrm{CsSb}$ : A HIGH AVERAGE CURRENT PHOTOCATHODE

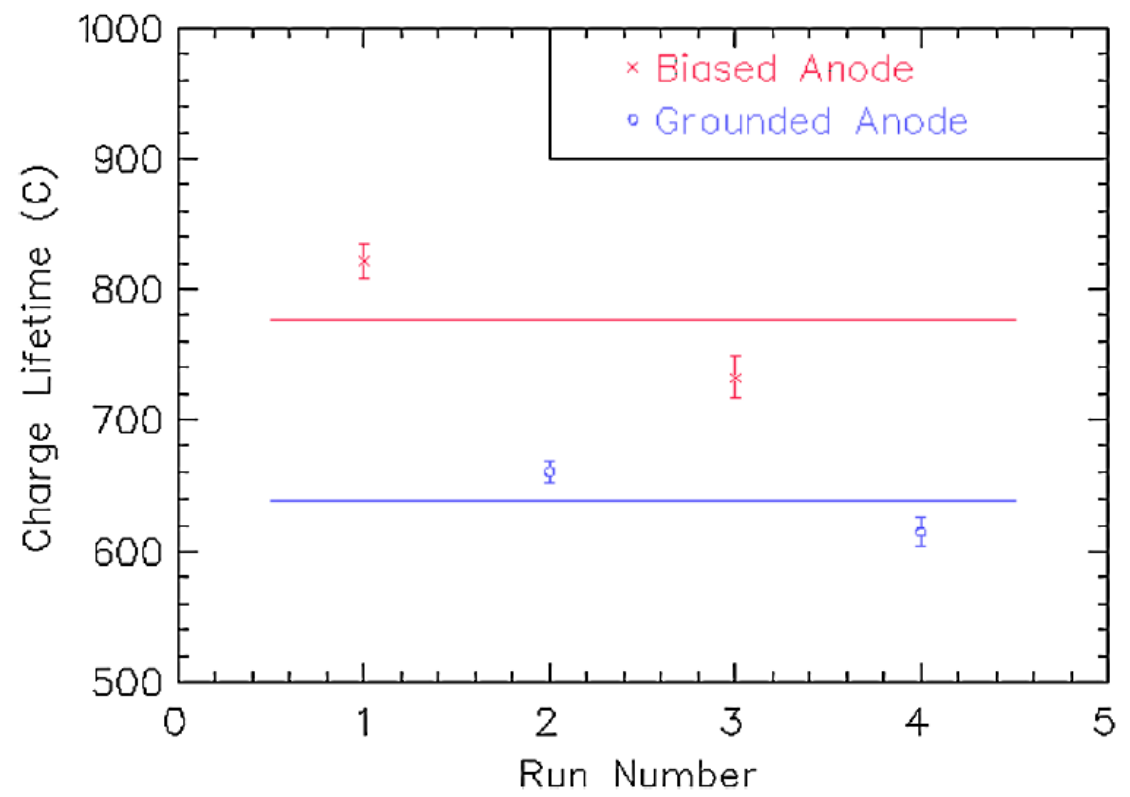

Figure 5.19: GaAs photocathode charge lifetime at $2 \mathrm{~mA}$ for alternately biased $(\mathrm{x})$ and grounded anode $(\bullet)$; the average for both the biased and grounded configurations is shown with horizontal bars. From Ref. [5.13].

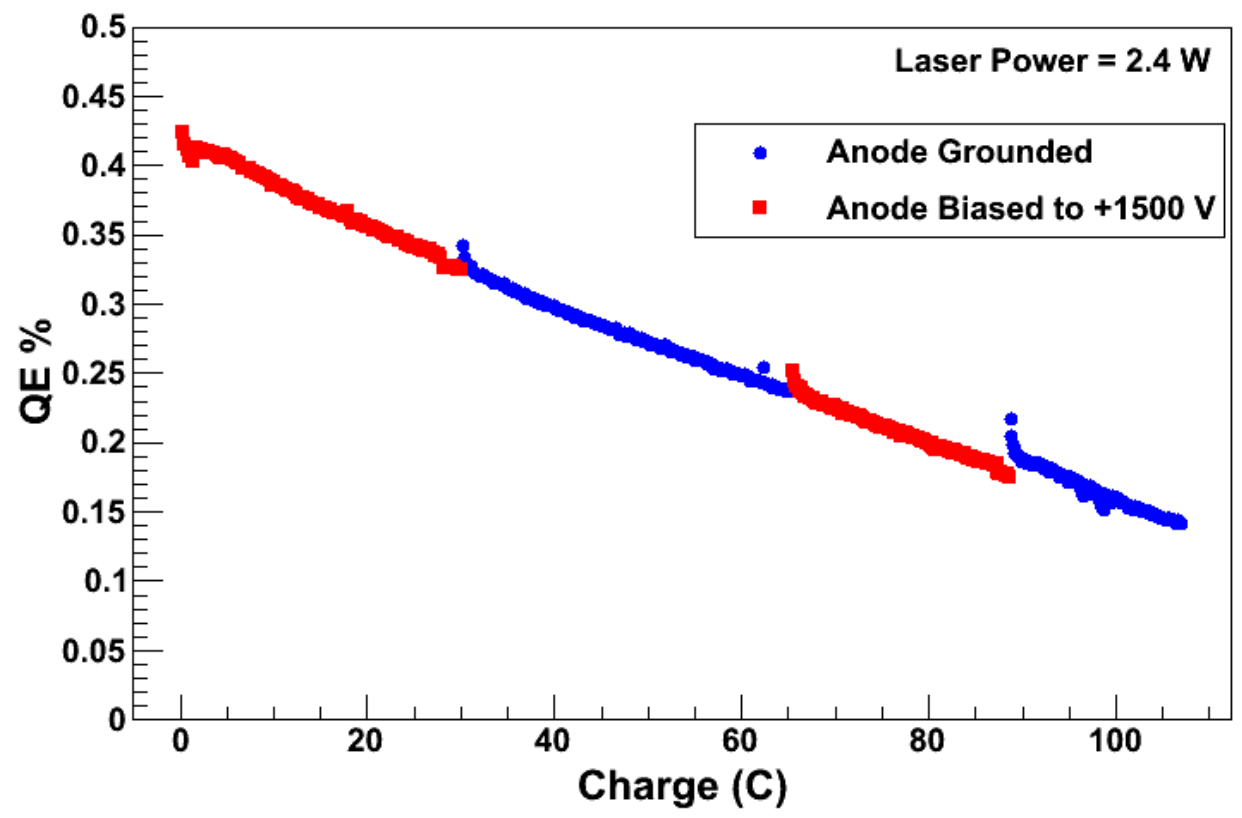

Figure 5.20: The $\mathrm{QE}$ at the electrostatic center of $\mathrm{K}_{2} \mathrm{CsSb}$ photocathode \#2 versus time at $10 \mathrm{~mA}$ average current and with the anode biased at $+1500 \mathrm{~V}(\boldsymbol{\bullet})$ and grounded $(\bullet)$ 


\subsubsection{Photocathode \#2: Laser Heating}

For the next test, another way to evaluate the effects of laser heating on the photocathode was devised that was different than the QE difference maps used on photocathode \#1. Beam was delivered to the dump using $532 \mathrm{~nm}$ light with a constant laser power of $0.2 \mathrm{~W}\left(1 \mathrm{~W} / \mathrm{mm}^{2}\right.$ (FWHM)), which produced initial beam current of approximately $1 \mathrm{~mA}$. Beam current was allowed to vary with changing QE, in contrast to the charge lifetime runs with photocathode \#1 in which the current was held constant. Over a sufficient time period, a clear QE evolution signature was observed. The QE quickly increased from 1.25 to $1.5 \%$ within minutes, and then continued to gradually increase to over $2 \%$ in 5 days (Figure $5.21 \mathrm{a})$. This run was then ended, the high voltage was turned off, and the photocathode was grounded. The laser beam was then moved to a fresh photocathode location $1.5 \mathrm{~mm}$ away. Laser light at the same power level as the previous run was re-applied to the photocathode and the QE was measured at a low-bias voltage $(300 \mathrm{~V})$ at $\sim 2$ hour time intervals. Each QE measurement at this stage took less than 5 minutes, and no beam was run between these measurements. The $\mathrm{QE}$ at low bias voltage was less than $\mathrm{QE}$ measured at high voltage due to the Schottky effect [5.28]. To aid in qualitative comparison between the high voltage field off/on condition, for both the charge lifetime and equivalent laser power heating runs, the normalized QE is plotted in Figure 5.21, which is simply the measured QE at each location over time compared to the original QE measured. Aside from this Schottky difference, both conditions resulted in similar QE evolution trends, including the rapid initial QE rise.

The laser beam was moved to another new location and beam was delivered to the dump, but with

a higher laser power, $\sim 2 \mathrm{~W}\left(10 \mathrm{~W} / \mathrm{mm}^{2}(\mathrm{FWHM})\right)$, which corresponded to $10 \mathrm{~mA}$ initial beam current. A slight increase in QE was observed during the first hour and then QE fell to a relatively small value over the course of ten hours (Figure 5.21b). The discontinuity in the graph at hour 5 was related to a slight mis-steering of the beam at the dump, likely due to one of the beamline viewers again accumulating charge from beam halo. The beam was re-steered to the dump without interrupting the beam delivery. 
After $\sim 10$ hours, beam delivery was halted, the photocathode was grounded, and the laser was moved to a fresh location. The QE was measured at one hour intervals under the same "no beam" conditions as before for the $1 \mathrm{~mA}$ equivalent run. Again, similar QE evolution signatures were observed between the trials with the beam actually extracted and with the beam not extracted from the photocathode. These measurements at high and low laser power/beam current serve as another indication that the QE of $\mathrm{K}_{2} \mathrm{CsSb}$ photocathodes at the location of beam extraction varies primarily due to chemical changes induced solely by the laser, and not due to ion bombardment, which seems to only definitively have affected the electrostatic center while beam was run in poor vacuum. In total, from all beam tests run on photocathode \#1, over $6000 \mathrm{C}$ were extracted from the photocathode.

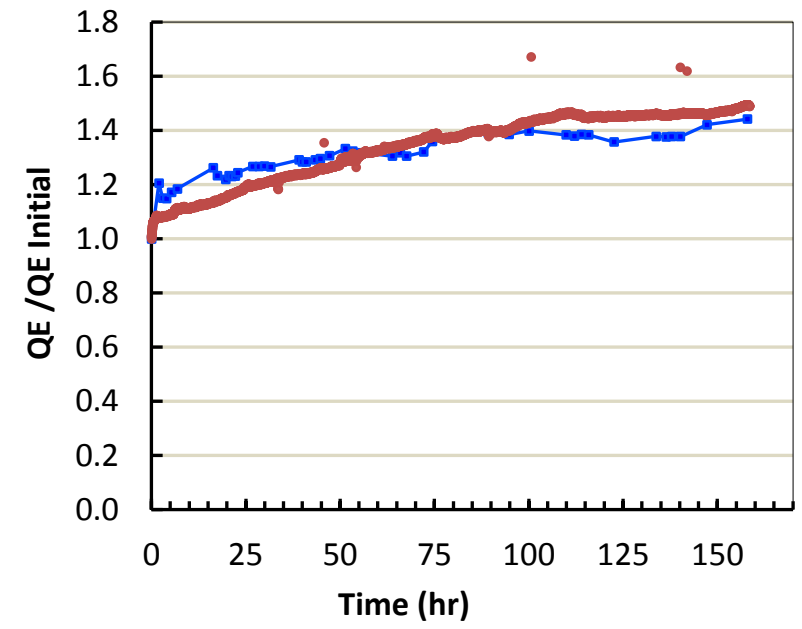

(a)

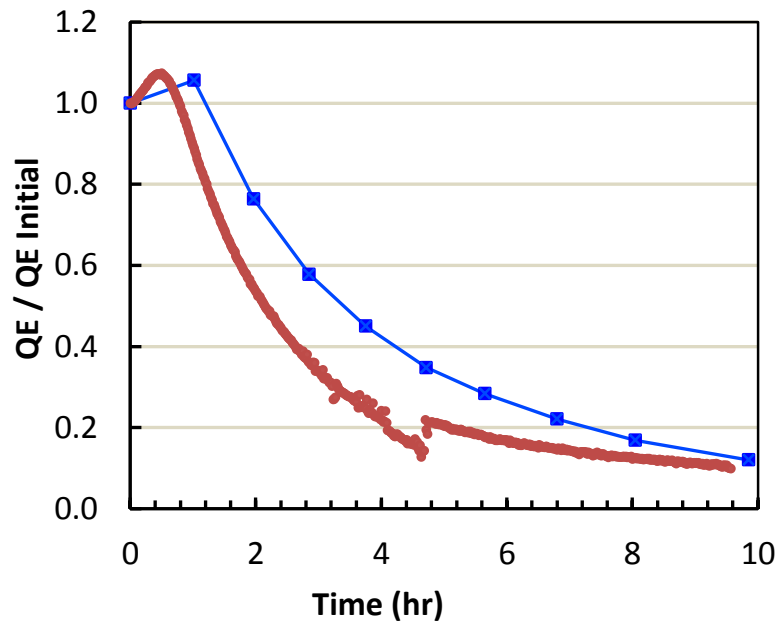

(b)

Figure 5.21: QE evolution at (a) $1 \mathrm{~mA}$ and (b) 10mA equivalent laser power. QE measured while delivering beam at $100 \mathrm{kV}(\boldsymbol{\square})$ and QE measured at low voltage during "laser heating" runs $(\bullet)$, with no high voltage and no beam delivered to the Faraday cup. Data points were connected to aid the eye.

\subsubsection{Photocathode \#2: Emittance}

While thermal emittance for a photoemission gun defines the highest brightness beam obtainable, the ITS was not equipped to measure thermal emittance, partly because its electron optic transfer matrices 
were not fully known from beam dump back to the photocathode due to rebuilds of gun chamber. In order to have some idea of the beam quality, the geometric emittance was measured. Geometric normalized root mean square (RMS) emittance was evaluated for beam produced from both photocathodes as a function of laser wavelength, laser beam size and photogun bias voltage using the previously mentioned solenoid scan technique [5.14]. In the manner describes in Section 5.1, the field strength of the last beamline solenoid in the ITS was varied in order to change the size of the electron beam, which was measured using a wire scanner located near the beam dump. Beam widths were extracted from Gaussian fits of the $\mathrm{x}, \mathrm{y}$, and $\mathrm{u}$ wire signals, with typically 10 scans made for each beam condition. A plot of beam width squared as function of solenoid current squared (Figure 5.22a) provides a measure of beam emittance.

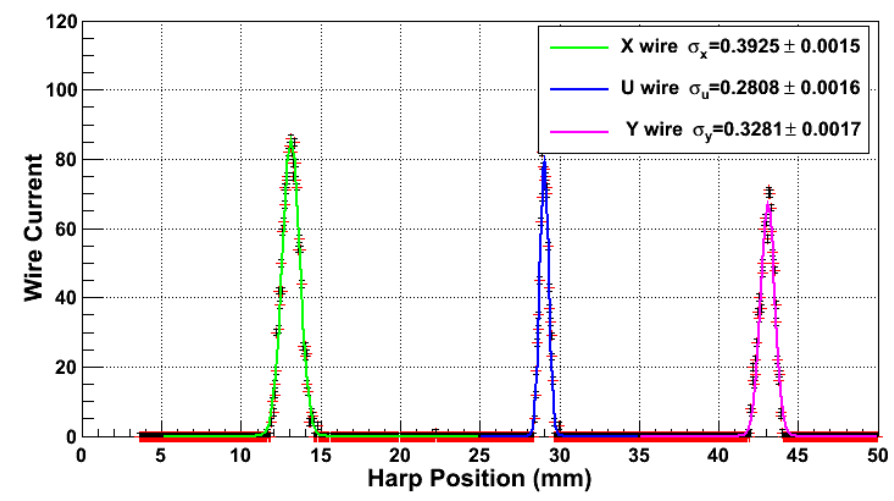

(a)

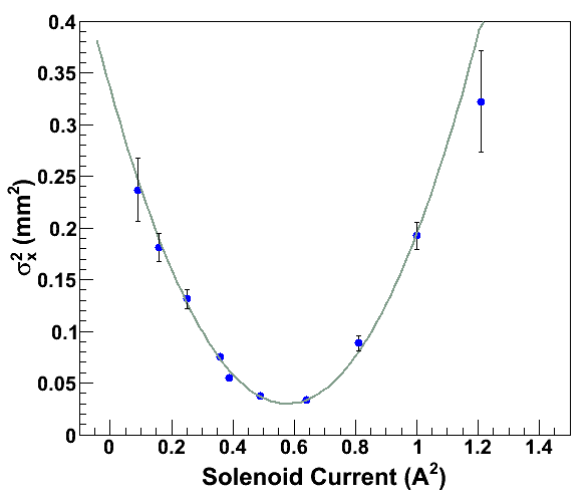

(b)

Figure 5.22: (a) An example of beam widths measured by a three-wire harp scanned across the beam path. (b) Gaussian beam widths in the $\mathrm{x}$ direction for different focusing solenoid field strengths, for beam extracted from the electrostatic center of photocathode \#2. The data were fit with a parabola to determine emittance, as described in the text.

To recap Section 5.1 in terms of the equipment used at the ITS, the geometric RMS emittance at any given point along the beamline is equal to $\varepsilon_{R M S}=\pi \sqrt{\operatorname{det} \sigma}=\pi \sqrt{\sigma_{11} \sigma_{22}-\sigma_{12}^{2}}$, where $\boldsymbol{\sigma}$ is the trace space beam matrix at that location. The sigma matrix at the harp, $\sigma_{\text {harp }}$, is defined by Eq. (5.14). Since 
CHAPTER 5: $\mathrm{K}_{2} \mathrm{CsSb}$ : A HIGH AVERAGE CURRENT PHOTOCATHODE

the beam radius at the harp, $\sigma_{x}$, for a given plane is equal to $\sqrt{\sigma_{11, \text { harp }}}$, one can fit the data in Figure 5.22a with a parabola of the given form in Eq. (5.6) to extract the beam pre-solenoid sigma matrix elements and therefore the geometric emittance. This process was repeated with different laser wavelengths, spot sizes, and beam energies. The effects due to laser beam size and beam energy were factored out using the equation $\epsilon_{n}=\beta \gamma \epsilon_{R M S} / w$, where $w$ is the RMS width of the laser, to calculate the normalized RMS emittance. Space charge effects were negligible as DC beam current was kept to $\sim 3 \mu \mathrm{A}$ for all measurements.

The emittance measurements using photocathode \#1 were performed after having completed extensive charge lifetime evaluation and after the vacuum event which severely reduced $\mathrm{QE}$ at the photocathode center. Measurements were made with the laser positioned approximately $2 \mathrm{~mm}$ from the electrostatic center, at 440 or $532 \mathrm{~nm}$ wavelengths, with two laser spot sizes, and with 100 or $200 \mathrm{kV}$ gun bias voltages. At $532 \mathrm{~nm}$, two laser spot sizes were used, $500 \mu \mathrm{m}$ and $1050 \mu \mathrm{m}$ FWHM, while at 4400 nm only a $1000 \mu \mathrm{m}$ FWHM laser spot was used. The measured average geometric normalized RMS emittance was $1.06 \pm 0.16 \mathrm{~mm} * \mathrm{mrad} / \mathrm{mm}$ at $440 \mathrm{~nm}$ and $0.91 \pm 0.08 \mathrm{~mm} * \mathrm{mrad} / \mathrm{mm}$ at $532 \mathrm{~nm}$.

For photocathode \#2, consideration was given to evaluating normalized RMS emittance before and after extracting significant charge, as it was possible that the emittance could change along with the QE change of the photocathode. In particular, the emittance was measured at the electrostatic center immediately upon installation of the photocathode within the photogun, as well as before and after an extended run at $1 \mathrm{~mA}(850 \mathrm{C}$ extracted), in order to determine if the geometric emittance degraded due to photocathode use. For each condition, the geometric emittance was the same, within errors. The average normalized RMS emittance was $0.72 \pm 0.07 \mathrm{~mm} * \mathrm{mrad} / \mathrm{mm}$ at $532 \mathrm{~nm}$. No attempt was made to relate measured geometric emittance to that at the photocathode (i.e., thermal emittance), as the electron optics of the ITS beamline are not entirely known. Even though the geometric beam emittance is inherently higher than the thermal emittance, the measured geometric normalized RMS emittance values are very similar to the thermal emittance values reported in reference [5.5]. 


\section{$5.4 \quad \mathrm{~K}_{2} \mathrm{CsSb}$ Surface Studies}

After the beam-based measurements of the two photocathodes that took place in the ITS, each photocathode was moved back into the vacuum transfer suitcase. The gate valve to the suitcase was closed, and the sealed vacuum chamber was moved to another building on site at TJNAF for evaluation of the surface morphology of the photocathodes using a scanning electron microscope (SEM) with an energy-dispersive x-ray spectroscopy (EDS) attachment. The suitcase was incompatible with the SEM, and as such the cathodes were not able to be moved into the SEM while in vacuum. To reduce the likelihood of photocathode oxidation, an argon-filled gloved bag was used to transfer the samples into the SEM. The transfer took approximately 2 minutes and once inside the SEM, samples were exposed to $\sim 10^{-6}$ Torr vacuum for the duration of measurements. In order to maximize the quality of the images, as well as the quality of the EDS spectra, an electron accelerating potential greater than $8 \mathrm{keV}$ was used for both photocathodes, which caused the electron penetration depth to be deeper than the thickness of the photocathode layer. The fluorescence strengths and detector efficiencies of the different elements were taken into account for all EDS composition measurements.

\subsubsection{Photocathode \#1}

After the charge lifetime and emittance measurements previously described were completed on the first photocathode, an attempt was made to quantify the $\mathrm{K}_{2} \mathrm{CsSb}$ lifetime as a function of vacuum level, as previous use [5.2] seemed to indicate a high lifetime, even in poor vacuum. The NEG pumps inside of the photogun chamber were heated to approximately $100{ }^{\circ} \mathrm{C}$ in order to liberate hydrogen in a relatively controlled manner; an inadvertent consequence was that the photocathode was heated as well. The QE rapidly dropped during this heating, and in an attempt to recover QE, a relatively small amount of Cs ( one monolayer) was applied to the entire surface. This attempt to recover QE failed, and these 
activities together reduced QE by a factor of three (Figure 5.23a). While the failed study likely had some impact on the chemical composition, surface science evaluation was still deemed worthwhile, especially as a means to further study the damage at the center of the cathode, which occurred as a result of running the beam into a flange for several hours.

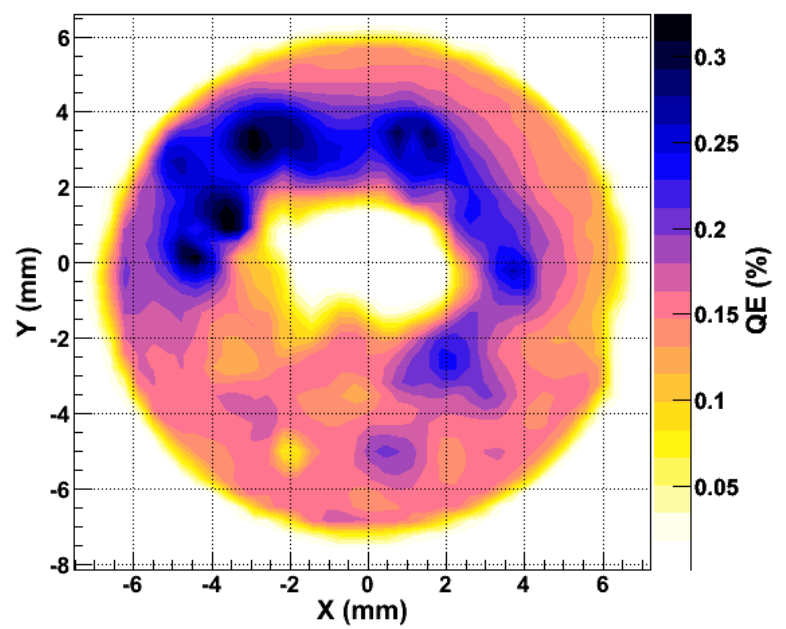

(a)

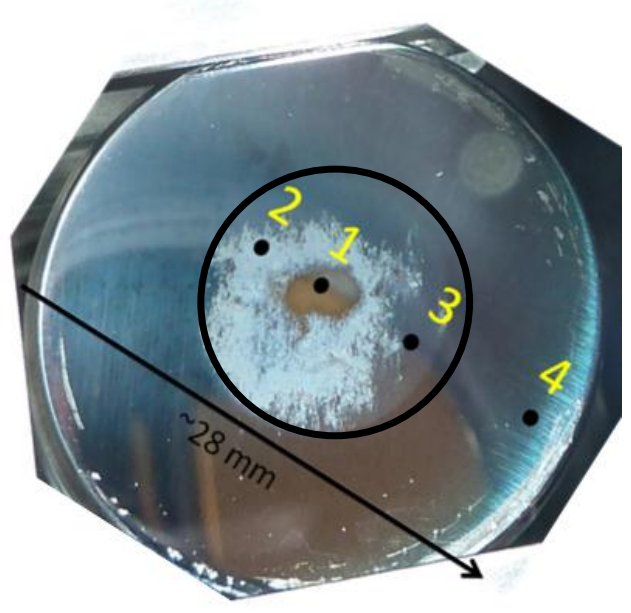

(b)

Figure 5.23: (a) QE scan of photocathode \#1 prior to transfer to the SEM and, (b) photograph of the entire photocathode showing the locations evaluated with SEM and EDS. Orientation between QE scan and photograph is approximately the same, with the QE scan taken inside the circled region in the photograph.

The surface topography and chemical composition were evaluated at four locations, as indicated in Figure 5.23b, using a $10 \mathrm{keV}$ electron beam. Figure 5.23b was taken after photocathode \#1 had been transferred into the suitcase, after all beam measurements were completed. These locations corresponded to the center of the photocathode (location \#1), which suffered nearly complete QE elimination during the vacuum event that occurred when beam was mis-steered into the beamline, to two locations representative of where most of the production beam delivery occurred (locations \#2 and \#3), and to a region that was protected from beam related effects, as it was located behind the cathode electrode (location \#4). An optical microscope allowed for closer visual inspection of the damaged center area, as well as the transition into other regions (Figure 5.24), note that these photographs were taken after removal from the SEM, and the slight coloring is due to oxidation in atmosphere. 


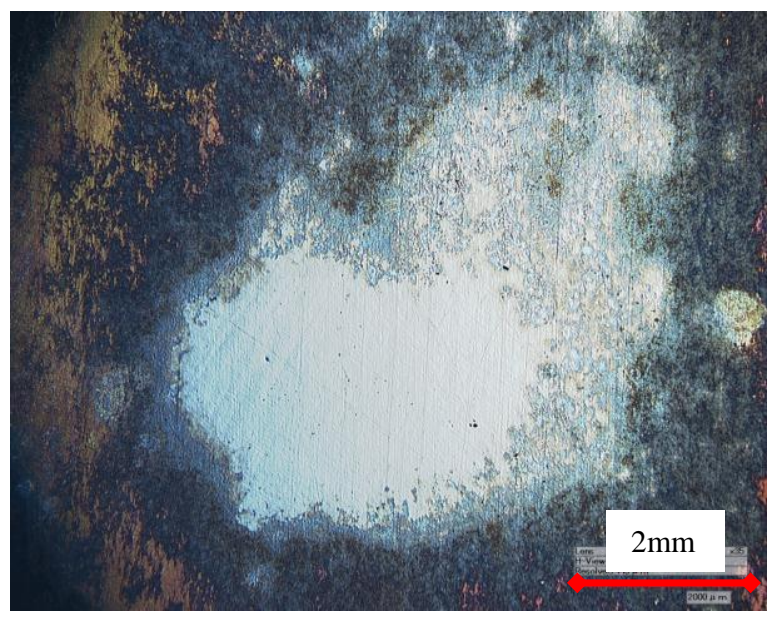

(a)

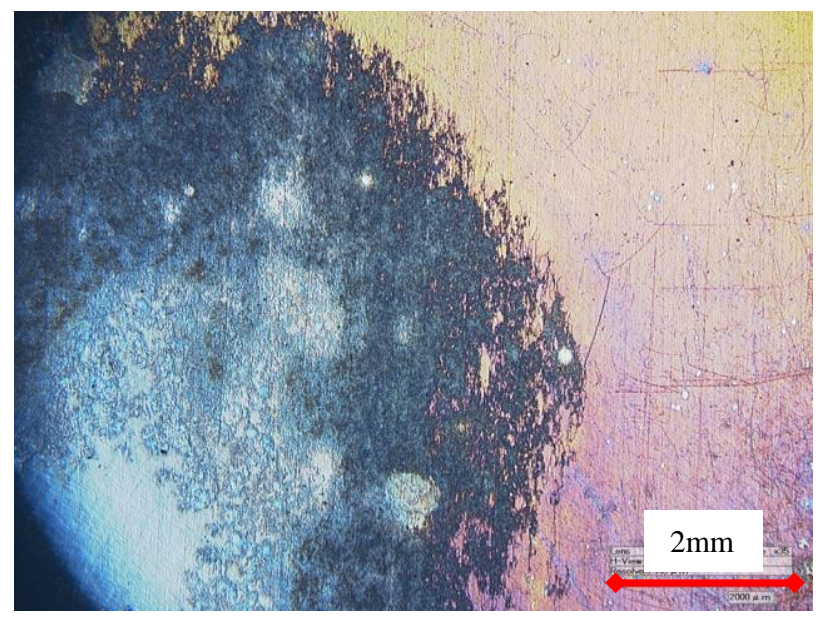

(b)

Figure 5.24: Photographs of photocathode \#1 after removal from the SEM, showing the central damaged area (a) and the transition away from the damage area $(b)$.

Figure 5.25 shows images of locations \#1, \#2, and \#3 at 400x magnification and locations \#2, \#3, and \#4 at 3000x magnification. Figure 5.26 shows the atom \% composition as determined by EDS x-ray data. From the EDS data, combined with the total lack of QE at location \#1 on the photocathode, it appears that the vacuum event was severe enough to sputter away the entire photocathode layer, leaving the stainless steel surface bare. Importantly, the rest of the cathode survived this major event, which would have completely destroyed the NEA surface condition of a GaAs photocathode and reduced the QE over the entire surface to zero. The 3000x image (Figure 5.25b) of location \#2 indicates islands of Cs, K, and $\mathrm{Sb}$ (the white flakes), with stainless steel and $\mathrm{Sb}$ in between the islands (the darker regions). Locations \#3 and \#4 show a more uniform photocathode layer, with some cracks exposing the stainless steel substrate underneath. Even though location \#2 appears to not be as uniform as location \#3, it still exhibits considerable QE, comparable even to that of location \#3. This behavior indicates that the QE of a $\mathrm{K}_{2} \mathrm{CsSb}$ photocathode is not overly sensitive to the uniformity of its surface. 
CHAPTER 5: $\mathrm{K}_{2} \mathrm{CsSb}$ : A HIGH AVERAGE CURRENT PHOTOCATHODE

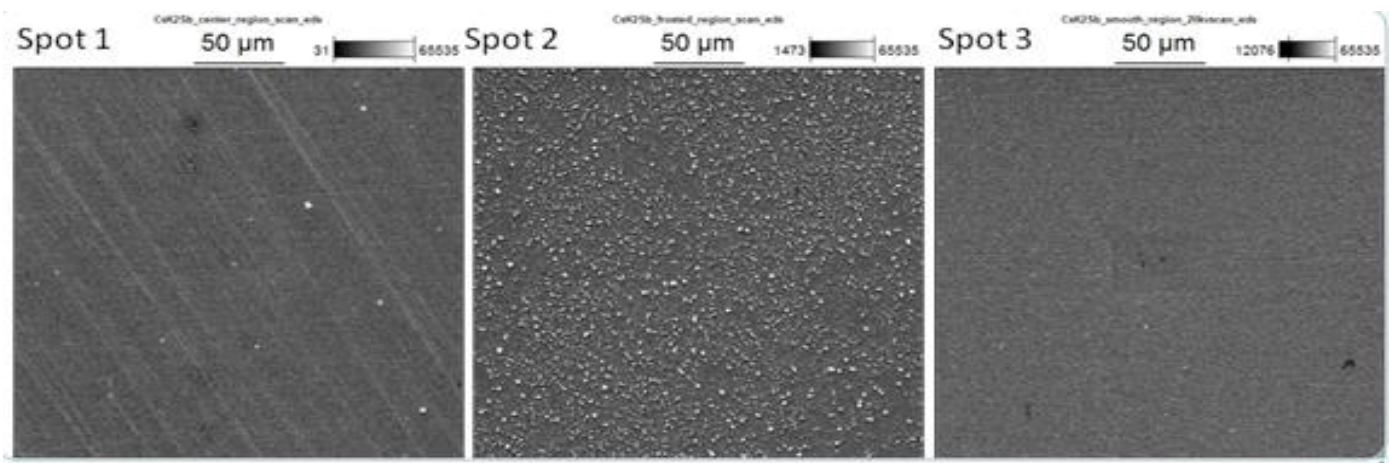

(a)
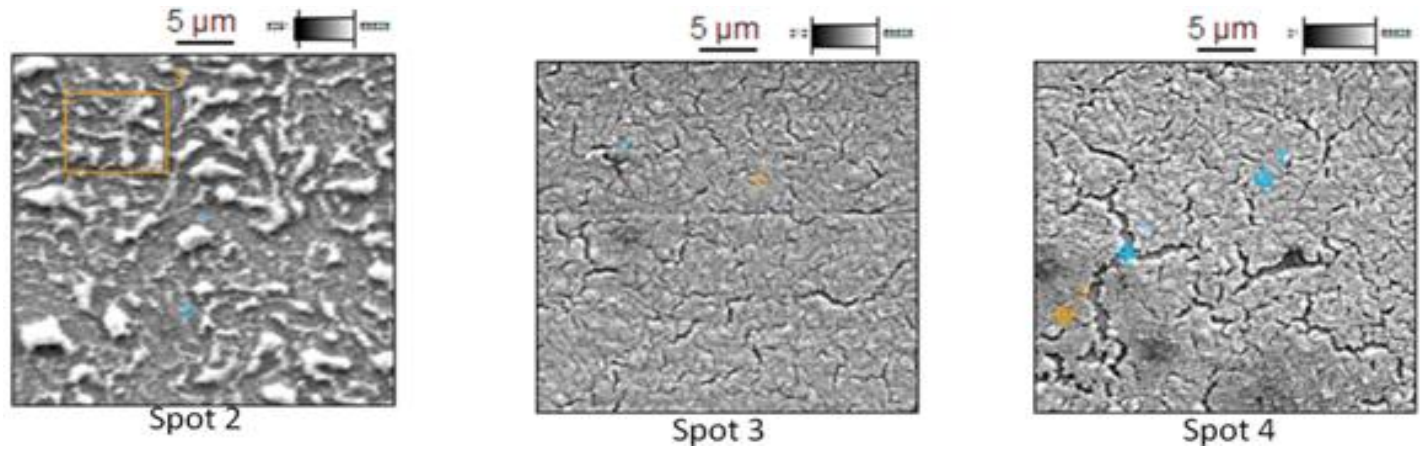

(b)

Figure 5.25: (a) Surface of photocathode \#1 at 400x magnification and (b) 3000x magnification.

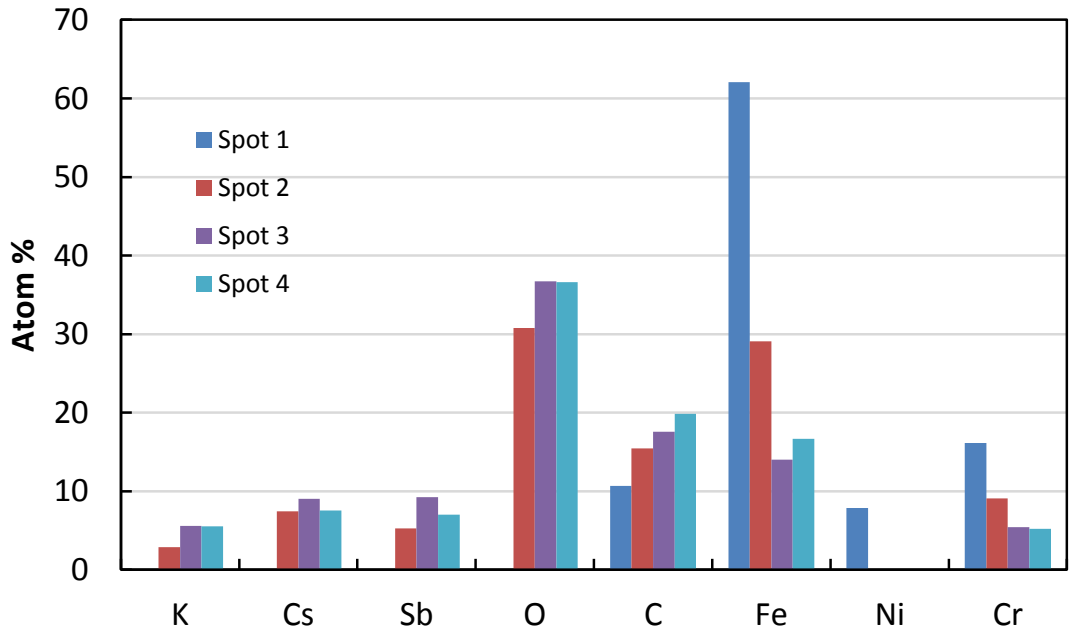

Figure 5.26: Chemical assay relative elemental species contribution by percent of total, as taken by EDS from SEM 400x images of locations 1-4 from photocathode \#1. 


\subsubsection{Photocathode \#2}

Photocathode \#2 was transferred into an SEM microscope in a similar manner to that of the first photocathode; it was moved into the vacuum suitcase, brought to another building, and then transferred inside an argon glove bag. Figure 5.27 shows a QE scan of photocathode \#2 (Figure 5.27a), as well as a photograph of the photocathode following all beam-based and laser-heating measurements inside the vacuum transfer suitcase (Figure 5.27). Visual inspection of the photocathode surface revealed distinct regions corresponding to both where beam was extracted and where the laser was used only to heat the sample, without extracting beam. These distinctive visual markers made it possible to study photocathode regions with known individual histories, which allowed the characteristics of the photocathode related to beam delivery and laser heating to be separated from nominally un-used locations.

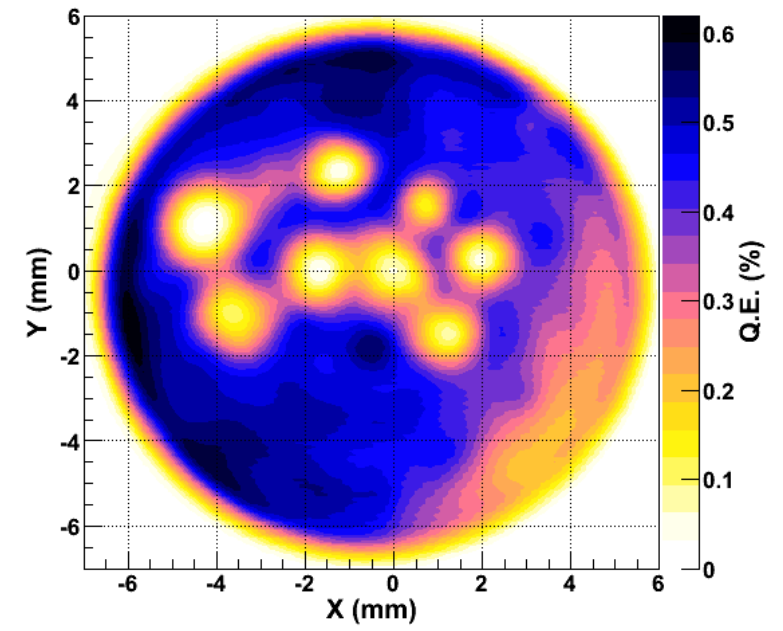

(a)

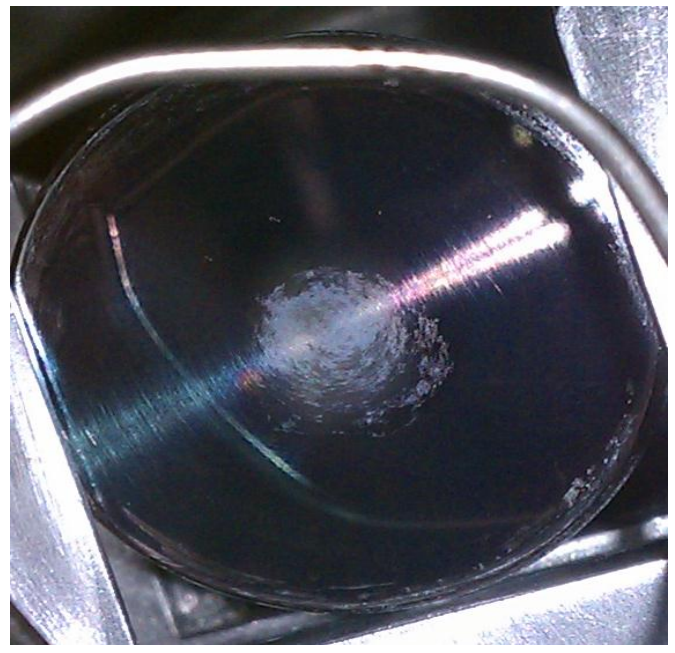

(b)

Figure 5.27: (a) QE scan and (b) a photograph of photocathode \#2 prior to transfer to the SEM. Orientation between QE scan and photograph are not related, with the QE scan taken inside the central region in the photograph.

After the photocathode puck was transferred into the SEM sample volume, problems in the apparatus required a vacuum vent with air. As a result, the photocathode surface became oxidized before any measurements could be made. Because this oxidation occurred over the entire surface, it still seemed 
CHAPTER 5: $\mathrm{K}_{2} \mathrm{CsSb}$ : A HIGH AVERAGE CURRENT PHOTOCATHODE

reasonable to comment on the relative $\mathrm{Cs}, \mathrm{K}$, and $\mathrm{Sb}$ content of the used and un-used locations, and as such, the SEM and EDS analyses were still undertaken. The influence of the laser on the surface can be seen in Figure 5.28, which is the 3,000x SEM image. Assuming that the initial photocathode surface was somewhat cracked, which follows from the method of film deposition combined with the observed quality of the surface of photocathode \#1 (Figure 5.25), it is apparent that the tighter focal spot in Figure 5.28b seems to have caused restructuring of the surface. While Figure 5.28a and b were taken while running/not running beam, respectively, because the $\mathrm{QE}$ evolutions as a function of time of photocathode \#2 were very similar under the running/not running beam conditions, this restructuring comparison between the SEM images can be made. In addition to the observed surface structure disparity between different size beams, a quantifiable change can be seen in the locations that were exposed to the laser to those that were not. EDS x-ray spectra were taken from 400x images centered at locations on the photocathode corresponding to either a beam run or laser heating location, or at nearby area that was not illuminated with the drive laser, in order to act as a control. Figure 5.29 shows the measured elemental composition from these scans. The amount of $\mathrm{Cs}$ and $\mathrm{K}$ is lower for those locations used for charge lifetime and laser heating experiments, whereas the amount of antimony is approximately the same for all four locations. Moreover, the decreased amount of oxygen at the laser illuminated sites is an indication that there is less $\mathrm{K}_{2} \mathrm{CsSb}$ to become oxidized at these sites. These measurements support the idea that QE degradation is associated with loss of photocathode material, in particular alkali material, and that this material loss was independent of beam generation. 


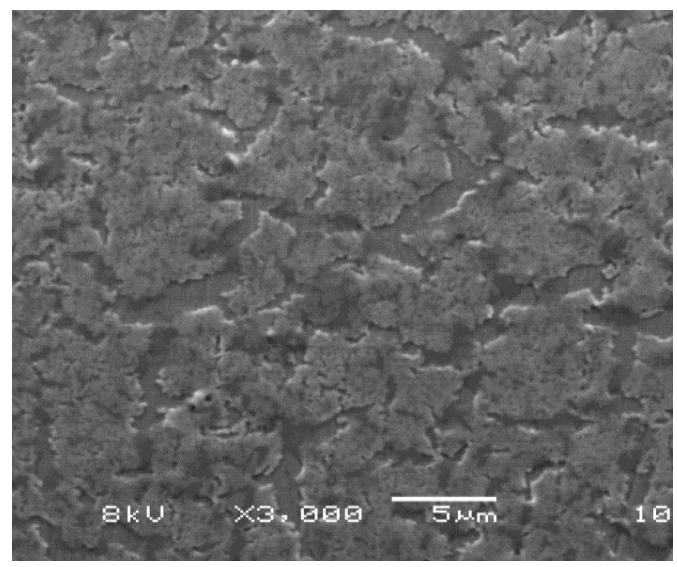

(a)

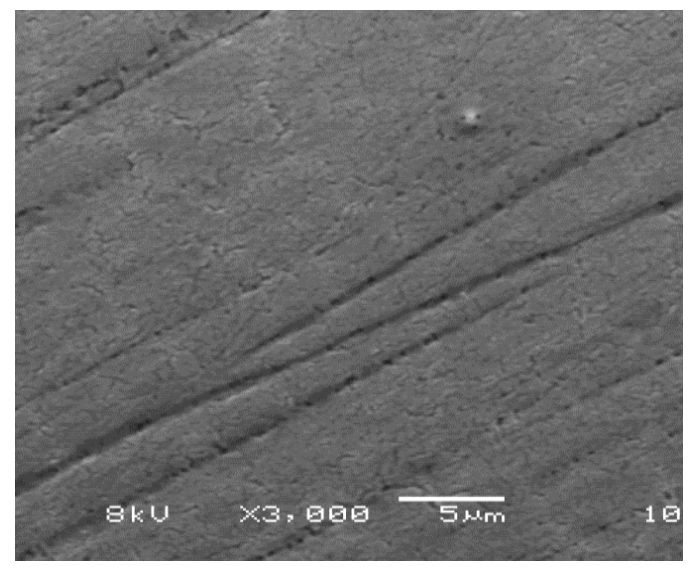

(b)

Figure 5.28: SEM images at 3000x magnification using an $8 \mathrm{keV}$ electron beam at photocathode locations that had been illuminated with $2 \mathrm{~W}$ laser light, in one case (a) while extracting beam at $10 \mathrm{~mA}$ using a laser spot $850 \mu \mathrm{m}$ FWHM and the other case, (b) heating the photocathode with a laser spot $500 \mu \mathrm{m}$ FWHM without generating beam.

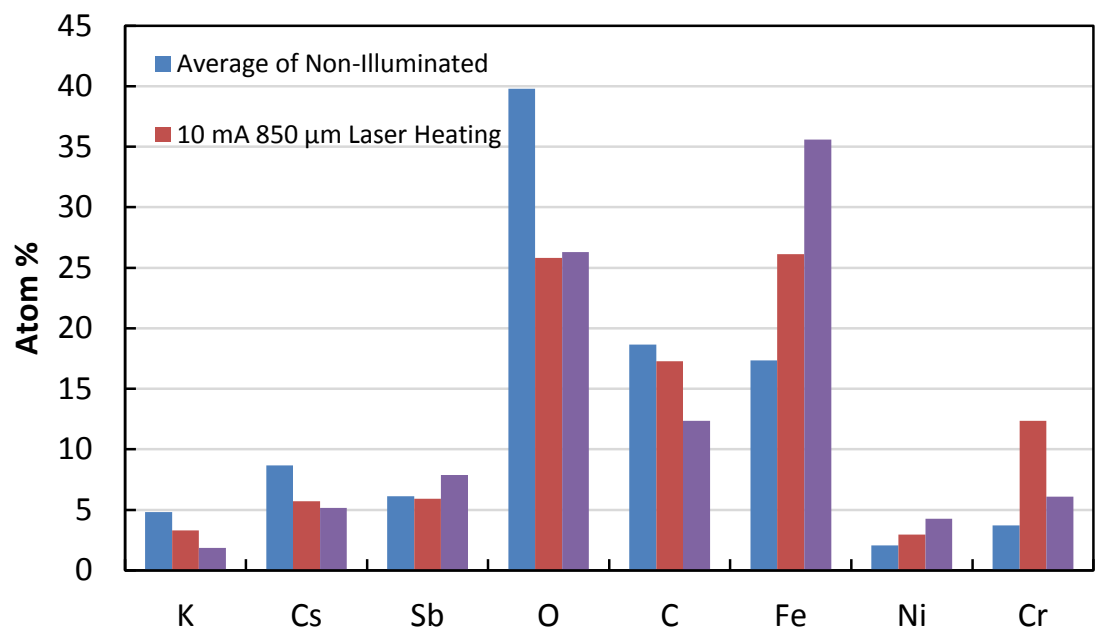

Figure 5.29: Chemical assay relative elemental species contribution by percent of total, as taken by EDS from SEM 400x from photocathode \#2. As noted, locations were illuminated with laser light while running/not running beam, or were un-used locations.

The SEM analysis of both photocathodes can be useful in finding an explanation for the different geometric RMS emittance of both photocathodes. While the emittance of photocathode \#1 was larger 
CHAPTER 5: $\mathrm{K}_{2} \mathrm{CsSb}$ : A HIGH AVERAGE CURRENT PHOTOCATHODE

than that of photocathode \#2, emittance \#1 was measured after the cathode was exposed to heavy use, as well as to relatively high vacuum conditions. Qualitatively, from Figure 5.25a and Figure 5.28a, the surface of the first photocathode rougher, which has previously been shown to lead to a higher geometric emittance [5.28][5.29].

\section{5 $\quad \mathrm{K}_{2} \mathrm{CsSb}$ Discussion and Thermal Simulation}

Both the beam and SEM based studies of $\mathrm{K}_{2} \mathrm{CsSb}$ seemed to indicate that the dominant influence on the QE evolution of the photocathode was due to the heating effect of the drive laser. The SEM showed that a high amount of laser intensity could restructure the surface; the EDS implied that the laser itself changed the chemical composition of the surface; and the beam studies indicated that at low laser intensity the local QE of the laser spot could be improved, while at high intensities the local QE decayed rapidly. In order to more quantifiably explain the behavior of the $\mathrm{QE}$ decay of the $\mathrm{K}_{2} \mathrm{CsSb}$ photocathodes, estimates of the photocathode temperature were made assuming $65 \%$ absorbed laser power (based on optical constants of $\mathrm{K}_{2} \mathrm{CsSb}$ and stainless steel from previous work [5.31],[5.32]) and using the commercial thermal modeling software ANSYS 14.0, as performed by R. Mammei and J. Feingold. The thermal analysis included a thin layer of stainless steel $(0.8 \mathrm{~mm})$ and the aluminum puck, which was in contact with the niobium cathode electrode and alumina inverted insulator. Between the stainless steel and aluminum puck were intervening layers of $\mathrm{Cu}(0.4 \mathrm{~mm})$ and $\mathrm{Ti}(0.4 \mathrm{~mm})$, which were required for the explosion bonding process that joined the stainless steel substrate to the rest of the puck. The $\mathrm{K}_{2} \mathrm{CsSb}$ itself was not included in the thermal model for several reasons. Firstly, calculations from the optical constants showed that the vast majority of laser power was absorbed by the stainless steel substrate due to the thinness, $50 \mathrm{~nm}$, of the $\mathrm{K}_{2} \mathrm{CsSb}$ layer. In addition, precise values of the thermal conductivity were unavailable for the structure of the thin film observed via SEM. Lastly, it was reasoned that because the surface area influenced by the laser was much larger than the thickness of the $\mathrm{K}_{2} \mathrm{CsSb}$, by 
a ratio of $7000: 1$, the heat conduction path would be through the stainless steel rather than spreading along the surface of the thin film. The model assumed excellent thermal contact between all of the components. The grounded end of the insulator, which was welded to a 10 inch stainless steel Conflat flange, was anchored at room temperature in the model. A diagram of the thermal model is shown in Figure 5.30a.

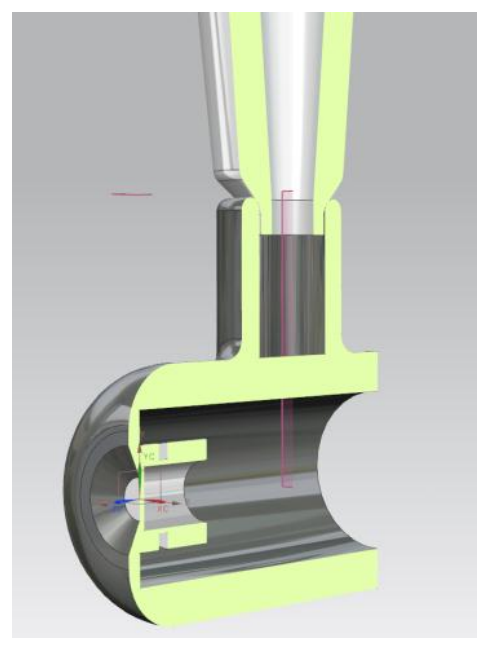

(a)

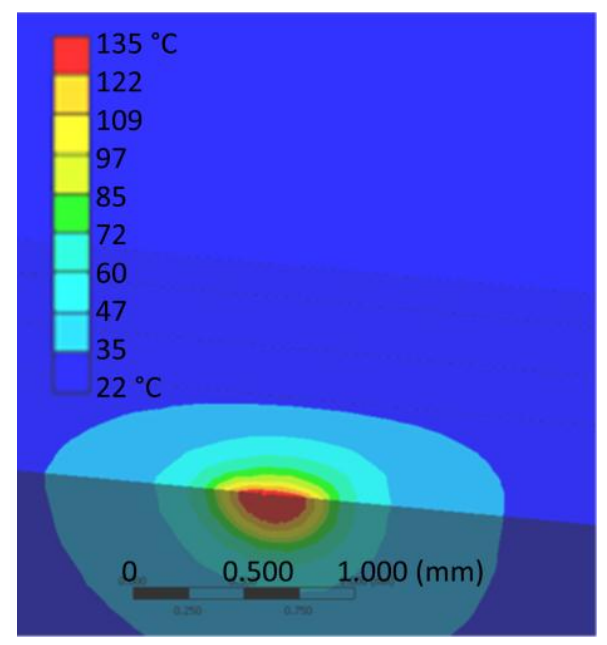

(b)

Figure 5.30: (a) Schematic view of the elements used in the ANSYS 14.0 thermal analysis. (b) Close-up cross sectional view of the photocathode and the stainless steel/aluminum puck with $1.3 \mathrm{~W}$ of laser light distributed over a $0.5 \mathrm{~mm}$ diameter region. The heat is primarily localized within the stainless steel, in which the temperature reaches $135^{\circ} \mathrm{C}$.

The thermal analysis indicates that most of the heat associated with the absorbed laser power stays within the thin photocathode and stainless steel layers, which is an effect due to the very poor thermal conductivity of stainless steel. Table 1 shows the maximum temperature reached for various incident laser powers with both 0.5 and $1 \mathrm{~mm}$ diameter laser spot sizes (FWHM). Photocathode heating experiments performed at BNL have shown that $\mathrm{K}_{2} \mathrm{CsSb}$ film is robust up to $\sim 100{ }^{\circ} \mathrm{C}$, but above this temperature the $\mathrm{QE}$ begins to drop, possibly because the film begins to disassociate. During photocathode growth, Cs is deposited with the substrate above $100{ }^{\circ} \mathrm{C}$, and while this may seem to be a contradiction, experiments on similar multi-alkali thin films indicate that dissociation temperatures will 
increase in the presence of a partial pressure of the alkali [5.33]. During a deposition, the relatively high partial pressure of Cs would increase the temperature required to dissociate the photocathode. For a 0.5 mm diameter laser spot, the thermal model suggests $100{ }^{\circ} \mathrm{C}$ is reached with only $1 \mathrm{~W}$ absorbed power (1.5 $\mathrm{W}$ incident power). This model seems to match the experimental results seen in the ITS. The observed charge lifetimes of the $\mathrm{K}_{2} \mathrm{CsSb}$ photocathodes were poor at currents $\sim 10 \mathrm{~mA}$ and higher, which required drive laser powers in excess of $1.5 \mathrm{~W}$; according to the model, this incident laser power would have been enough to damage the photocathode material, as $2 \mathrm{~W}$ of incident laser power at a $0.5 \mathrm{~mm}$ FWHM laser spot should drive the localized temperature to $135{ }^{\circ} \mathrm{C}$, far above the disassociate temperature. During the experimental $10 \mathrm{~mA}$ charge lifetime measurement on photocathode \#2, the QE dropped by $80 \%$ over 100 hours, but during the $1 \mathrm{~mA}$ run, which used $0.2 \mathrm{~W}$ of laser power, the QE showed no sign of decrease over 160 hours. The disparity in charge lifetime suggests that a temperature threshold between $40{ }^{\circ} \mathrm{C}$ and $130{ }^{\circ} \mathrm{C}$ exists, beyond which $\mathrm{K}_{2} \mathrm{CsSb}$ begins to decay. The thermal model and speculation that $\mathrm{K}_{2} \mathrm{CsSb}$ dissociates at elevated temperatures is also supported by the SEM/EDS measurements presented in the previous section, in which the concentrations of alkali elements at laser illuminated sites were found to be lower than those concentrations at un-used photocathode locations. While the SEM, charge lifetime, and laser heating experiments discussed here indicate a chemical disassociation brought about from the laser heating the stainless steel substrate, a dedicated heating experiment in which the chemical composition was monitored, with possibly an X-ray photoelectron spectrometer, would be required to more quantitatively define this thermal threshold.

In order to be a very high average current photocathode, $\mathrm{K}_{2} \mathrm{CsSb}$ will need to be robust under high laser powers, which is not a quality seen in these experimental results. The thermal model indicated that the stainless steel substrate was the cause of the high surface temperatures, and as a possibly fix, the same thermal analysis was performed using a molybdenum puck/substrate, which has a thermal conductivity $\sim 10$ times better than stainless steel. Molybdenum was chosen over copper or aluminum as a substrate to model, as $\mathrm{K}_{2} \mathrm{CsSb}$ grown on molybdenum has been shown to result in a higher $\mathrm{QE}$ 
CHAPTER 5: $\mathrm{K}_{2} \mathrm{CsSb}$ : A HIGH AVERAGE CURRENT PHOTOCATHODE

photocathode than $\mathrm{K}_{2} \mathrm{CsSb}$ /copper and $\mathrm{K}_{2} \mathrm{CsSb}$ /aluminum combinations [5.24]. This new analysis found that up to $7 \mathrm{~W}$ laser power could be directed at the photocathode while maintaining temperature below $100{ }^{\circ} \mathrm{C}$. According to the modeling, using the $1.3 \mathrm{~W}$ of laser power required for $10 \mathrm{~mA}$ of beam from a $\sim 1 \% \mathrm{QE} \mathrm{K}_{2} \mathrm{CsSb}$ photocathode would only raise the temperature to $40{ }^{\circ} \mathrm{C}$, which is a temperature that allows for stable $\mathrm{QE}$ operation. Thus, a $\mathrm{K}_{2} \mathrm{CsSb}$ photocathode with $12 \% \mathrm{QE}$ grown on a good thermal conductor, like molybdenum, could provide $100 \mathrm{~mA}$ beam current without suffering the QE decay associated with laser thermal-induced chemical decomposition.

Table 5.1: Maximum simulated temperature at the illuminated location for a given absorbed laser power for the used $\mathrm{K}_{2} \mathrm{CsSb}$, stainless steel, and aluminum puck as well as a $\mathrm{K}_{2} \mathrm{CsSb}$, molybdenum puck.

\section{Maximum Temperature: \\ Stainless steel substrate with aluminum puck \\ Maximum Temperature: \\ Molybdenum puck}

\begin{tabular}{|c|ccccc|ccc|}
\hline $\begin{array}{c}\text { Absorbed Laser } \\
\text { Power }\end{array}$ & $0.2 \mathrm{~W}$ & $1.0 \mathrm{~W}$ & $1.3 \mathrm{~W}$ & $2 \mathrm{~W}$ & $2.5 \mathrm{~W}$ & $1.3 \mathrm{~W}$ & $3 \mathrm{~W}$ & $5 \mathrm{~W}$ \\
$\begin{array}{c}\text { Laser Spot Size: } \\
0.5 \mathrm{~mm}\end{array}$ & $40{ }^{\circ} \mathrm{C}$ & $108^{\circ} \mathrm{C}$ & $135^{\circ} \mathrm{C}$ & $173{ }^{\circ} \mathrm{C}$ & & $42^{\circ} \mathrm{C}$ & $67{ }^{\circ} \mathrm{C}$ & $98{ }^{\circ} \mathrm{C}$ \\
\hline $\begin{array}{c}\text { Laser Spot Size: } \\
1 \mathrm{~mm}\end{array}$ & & & $78^{\circ} \mathrm{C}$ & & $129^{\circ} \mathrm{C}$ & $36^{\circ} \mathrm{C}$ & & \\
\hline
\end{tabular}

\section{$5.6 \quad \mathrm{~K}_{2} \mathrm{CsSb}$ Conclusions}

Two $\mathrm{K}_{2} \mathrm{CsSb}$ photocathodes were grown at Brookhaven National Lab and transported $\sim 450$ miles to Jefferson Lab inside a compact UHV apparatus, where they were installed inside a DC high voltage photogun previously used to evaluate GaAs photocathodes. Charge lifetime measurements were performed at currents up to $20 \mathrm{~mA}$ using both 532 and $440 \mathrm{~nm}$ laser light with bias voltages of both 100 and $200 \mathrm{kV}$. A total charge of approximately 6000 Coulombs was extracted from both photocathodes combined.

Photocathode \#1 initially exhibited poor charge lifetime at $532 \mathrm{~nm}$. However, charge lifetime at $440 \mathrm{~nm}$ was much better, with no observed QE decay at beam currents up to $5 \mathrm{~mA}$ over a 24 hour time 
CHAPTER 5: $\mathrm{K}_{2} \mathrm{CsSb}$ : A HIGH AVERAGE CURRENT PHOTOCATHODE

period. An inadvertent beam steering accident lasting 1.5 hours resulted in the complete removal of photocathode material at the electrostatic center, but left useable material at the periphery of the photocathode. Subsequent charge lifetime measurements at $532 \mathrm{~nm}$ showed completely different behavior to the poor results seen previously, with markedly improved charge lifetime at milliampere beam currents. It is unclear what aspect of the beam-strike condition resulted in improved photocathode performance from the remaining photocathode material.

Charge lifetime at $532 \mathrm{~nm}$ was very good at the outset for photocathode \#2 at $1 \mathrm{~mA}$ current, but poor at $10 \mathrm{~mA}$. Measurements taken to identify causes of the QE decay indicated that under normal beam delivery conditions, including UHV vacuum, ion bombardment did not play a significant role. The QE behavior was directly linked to chemical changes associated with laser heating, and a thermal model indicated that a photocathode grown atop a thin stainless steel substrate can reach high temperature (> $\left.100{ }^{\circ} \mathrm{C}\right)$ with modest levels $(\sim 1 \mathrm{~W})$ of laser power. With this knowledge, using a stainless steel substrate, although it has high initial QE, is a poor choice for high current $\mathrm{K}_{2} \mathrm{CsSb}$ operations.

The normalized geometric RMS emittance was measured using the solenoid scan technique at laser wavelengths of both 440 and $532 \mathrm{~nm}$ with both 100 and $200 \mathrm{kV}$ bias voltages. Measurements on photocathode \#1 were performed after an extensive beam measurement program. For photocathode \#2, the emittance was measured before any charge lifetime measurements, and the normalized geometric RMS emittance for photocathode \#2 was found to lower than that of photocathode \#1. Surface analysis of photocathode \#1 suggested that the increased emittance could be due to a rough surface and non-uniform photocathode composition. Emittance measurements of the beam generated from photocathode \#2 are in reasonable agreement with previously published values. In addition there was no change in the emittance from photocathode \#2 after extracting a significant amount of charge at milliamp currents.

The topographies of the two photocathodes following their tests inside the photogun were markedly different from each other, as well as different across each individual photocathode surface. For photocathode \#1, which was subjected to extreme conditions during the beam striking the vacuum flange, 
CHAPTER 5: $\mathrm{K}_{2} \mathrm{CsSb}$ : A HIGH AVERAGE CURRENT PHOTOCATHODE

even though no photocathode material remained at the center of the puck, the edges of the photocathode still surprisingly provided reasonable amounts of beam.

EDS data showed that the alkali content at the photocathode locations which had been illuminated with laser light, whether or not electron beam was extracted, was diminished compared to photocathode locations that had not been illuminated with laser light.

There still exist several interesting questions associated with the use of $\mathrm{K}_{2} \mathrm{CsSb}$ photocathodes for high current accelerator applications. The first question that could be addressed is a better definition of the heat threshold that beyond which, the QE rapidly decays. This question could possibly be answered by heating a virgin $\mathrm{K}_{2} \mathrm{CsSb}$ cathode directly, and observing crystalline structure and QE changes. Also, the exact influence of ion bombardment is of yet unclear. This experiment indicates that in vacuum environments $\sim 10^{-12}$ Torr, ion bombardment does not normally play a significant role in the QE evolution of $\mathrm{K}_{2} \mathrm{CsSb}$; however, at higher pressures, such as that created during the beam steering accident, ion bombardment sputtered the photocathode film completely away from the electrostatic center. In addition to investigating the effects of ion bombardment, photocathode surface morphology deserves further study. Even to the naked eye, the surface of both photocathodes was noticeably different following tests inside the photogun, and detailed SEM images clearly indicated significant morphological differences between unused regions, locations where beam was extracted, and locations that were merely illuminated with laser light. The factors that affect these changes are not well defined, and neither is the degree to which these morphological changes impact photocathode lifetime and beam quality. Future beam-based experiments will surely serve to improve the understanding of this important photocathode material. 


\section{Conclusion}

This dissertation presented several studies of two different types of photocathodes used at electron accelerators: GaAs and $\mathrm{K}_{2} \mathrm{CsSb}$. In order to study photocathodes offline of the main CEBAF machine at JLab, a new style of retarding field micro-Mott polarimeter was commissioned. The polarimeter had the benefits of rapid sample changes, ease of construction, and small size, with an operational range of 5 to $30 \mathrm{kV}$. Using a cross comparison to JLab's CEBAF \% MeV Mott polarimeter, the effective Sherman function, $S_{\text {eff }}$, was determined to be $0.201 \pm 0.004$ at $20 \mathrm{kV}$ and $\Delta \mathrm{E}=0$. While $S_{\text {eff }}$ is comparable to current other current polarimeters, the efficiency, and consequently the figure-of-merit, was significantly lower than comparable designs. Although the cause of this low efficiency is not entirely understood, the operational capabilities of this new style polarimeter are still high enough to allow for useful operations in prequalifying photocathode materials before insertion into the main CEBAF photogun and in conducting offline research programs.

For the first research program conducted with the polarimeter, the polarization of electrons emitted via two-photon absorption in GaAs was studied, as some earlier work had indicated that two-photon absorption could lead to polarization values $>50 \%$. While there had been past contradictory theoretical and experimental results on the expected degree of electron polarization, prior to this work no direct measurement had been made of the polarization of the electron beam photoemitted due to two-photon absorption. Using the linear relationship of QE to incident laser intensity, photocurrent was shown to be entirely due to two-photon absorption while the GaAs was illuminated with $1560 \mathrm{~nm}$ light. From bulk GaAs, the polarization at $1560 \mathrm{~nm}$ was approximately half the value at $780 \mathrm{~nm}(16.8 \pm 0.4 \%$ to $33.4 \pm 0.8 \%$, respectively.) Limiting the active thickness of the GaAs to thinner values, the one- and twophoton polarization converged to approximately the same value ( 43\% to $40 \%$, respectively.) This convergence indicates that the maximum polarization obtainable via two-photon absorption is close to 
CHAPTER 6: CONCLUSION

that from one-photon absorption, only 50\%. In addition to measuring polarization, two other properties of GaAs were experimentally determined. The two-photon absorption coefficient, $\beta$, was measured to be $1.6 \pm 0.5 \mathrm{~cm} / \mathrm{GW}$, with the spin relaxation length, $l_{s p}$, measured to be $2.810^{-5} \mathrm{~cm}$. Because the twophoton polarization is only $50 \%$ and the two-photon QE is a factor $10^{5}$ times smaller than the one-photon QE, the program concluded that two-photon absorption is not a viable means of production of highly spin polarized electron beams.

While CEBAF currently requires polarized electron beam, many other accelerators have no such constraint. Looking towards future projects, the CIS at JLab, and other groups, will require a photocathode that is capable of meeting the extremely high average beam current demands of proposed machines (light sources, energy recovery linacs and electron cooling machines). Because of past success, $\mathrm{K}_{2} \mathrm{CsSb}$ is a popular photocathode choice for high current demands, and it is currently being studied by many groups, including the CIS.

Using photocathodes grown at BNL, systematic charge lifetime measurements, at currents up to $20 \mathrm{~mA}$, were performed with $\mathrm{K}_{2} \mathrm{CsSb}$ using a DC high voltage photogun, which had previously evaluated GaAs photocathodes. While the first $\mathrm{K}_{2} \mathrm{CsSb}$ photocathode initially displayed poor charge lifetime with incident light at $532 \mathrm{~nm}$, the lifetime at $440 \mathrm{~nm}$ was better, and after a vacuum event, the lifetimes at both $440 \mathrm{~nm}$ and $532 \mathrm{~nm}$ were very high. For both the first and second photocathodes, a dependence of lifetime with the intensity of incident drive light was observed. Beam tests involving biasing the anode, turning high voltage off/on, and varying the laser spot size indicate that at a vacuum pressure of $2 \times 10^{-12}$ Torr, the dominate $\mathrm{QE}$ decay mechanism of $\mathrm{K}_{2} \mathrm{CsSb}$ is due to laser heating, as opposed to ion bombardment. Thermal modeling indicated that the stainless steel substrate used during photocathode growth reached high temperature $\left(>100{ }^{\circ} \mathrm{C}\right)$ with modest levels $(\sim 1 \mathrm{~W})$ of laser power and EDS data showed a decrease in alkali content from locations on the photocathode that had been illuminated. The loss of alkali material due to a heated substrate is the likely cause of the observed dependence of charge lifetime with laser intensity. For future use, the stainless steel substrate on which the $\mathrm{K}_{2} \mathrm{CsSb}$ was grown 
should be replaced with molybdenum or other material highly conductive to heat. Even with a change in substrate, high current operation of the photocathode may require the use of larger beam spots than the $850 \mu \mathrm{m}$ and smaller spot sizes used in these measurements, although future work is needed to understand the exact thermal limitations of this photocathode. In conclusion, although $\mathrm{K}_{2} \mathrm{CsSb}$ showed sensitivity to laser heating, the very long charge lifetimes obtained using lower laser intensities indicate a very bright future for its use in high average current, high brightness machines. 


\section{Bibliography}

[1.1] J. Kessler, Polarized Electrons (Springer-Verlag, Berlin 1985)

[1.2] W.E.Spicer and A. Herra-Gomez, SLAC-PUB 6306 (1993), presented at the 1993 SPIE International Symposium on Imaging and Instrumentation, San Diego

[1.3] T. Maruyama, et. al., Phys. Rev. B 46, 4261 (1992)

[1.4] H. Aoyagi, et. al., Phys. Lett. A 167, 415 (1992)

[1.5] Aulenbacher, et. al., Nucl. Instrum. and Meth. A 391, 498 (1997)

[1.6] W. Von Drachenfels, et. al., AIP Conf. Proc. 675, 1053 (2003)

[1.7] F. Zhou, et. al., AIP Conf. Proc. 1149, 992 (2009)

[1.8] M. Poelker AIP Conf. Proc. 1149, 174 (2009)

[1.9] T. Matsuyama, et. al., in $4^{\text {th }}$ Pacific Rim Conf. on Lasers and Electro-Optics 164 (2001)

[1.10] P. Hartmann, et. al. Nucl. Instr. Meth. A 379, 15 (1995)

[2.1] E.J. Montgomery Doctoral Thesis, University of Maryland, College Park (2010)

[2.2] K. Aulenbacher et al., SLAC Report No. 432 (1993)

[2.3] M.L. Stutzman and J. Grams, AIP Conf. Proc. 11491032 (2008)

[2.4] R.L. Bell and W.E. Spicer, Proc. Of the IEEE 58, 1788 (1970)

[2.5] J.S. Blakemore, et al., J. Appl. Phys. 53, R123 (1982)

[2.6] R.L. Bell, Negative Electron Affinity Devices, (Clarendon Press, Oxford 1973)

[2.7] S.M. Sze, Physics of Semiconductor Devices, (John Wiley \& Sons, New York 1981)

[2.8] P. Saez, Doctoral Thesis, Stanford University (1997)

[2.9] L.W. James, et al., J. Appl. Phys. 42, 4976 (1971)

[2.10] J.S. Blakemore, et al., J. Appl. Phys. 53, R123 (1982) 
CHAPTER 7: BIBLIOGRAPHY

[2.11] R.K. Willardson and A.C. Beer - Editors, Semiconductors and Semimetals, J.S. Escher, Ch. 3:NEA Semiconductor Photoemitter (Academic Press, New York 1981)

[2.12] A. Yariv, Optical Electronics (Holt, Rinehart and Winston, New York 1985)

[2.13] D.T. Pierce and F. Meier, Phys. Rev. B13 5484 (1976)

[2.14] F. Meier and B.P. Zakharchenya - Editors, Optical Orientation, G.E. Pikus and A.N. Titkov, Ch.3:Spin Relaxation under Optical Orientation in Semiconductors, (North-Holland 1984)

[2.15] G. Fishman and G. Lampel, Phys. Rev. B16 820 (1977)

[2.16] M. Erbudak and B. Reihl, Appl. Phys. Lett. 33584 (1978)

[3.1] N.F. Mott, Proc. Of the Roy. Soc. A 124, 425 (1929) and 135, 429 (1932)

[3.2] T.J. Gay, F.B. Dunning, et al., Rev. Sci. Instrum. 63, 114 (1992)

[3.3] T.J. Gay, F.B. Dunning, et al., Rev. Sci. Instrum. 63, 1635 (1992)

[3.4] SAES Alkali Metal Dispenser, Cs, from SAES Getters

[3.5] Physical Electronics Ti-Tan CV ion pumps and SAES ST707 getter material, WP750 configuration, removed from insulated housing and set in bottom of chamber

[3.6] Keithley 485 Autoranging Picoammeter

[3.7] SIMION charged particle trajectory modeling software

[3.8] LabVIEW Data Acquisition Software, from National Instruments.

[3.9] H.M. Al-Khateeb, et al., Rev. Sci. Instrum. 70, 3882 (1999)

[3.10] T.G. Anderson, et al., Rev. Sci. Instrum. 72, 2923 (2001)

[3.11] L.G. Gray, et al., Rev. Sci. Instrum. 55, 88 (1984)

[3.12] L.A. Hodge, et al., Rev. Sci. Instrum. 50, 5 (1979)

[3.13] Channel Electron Multiplier type KBL 18RS, from Dr. Sjuts Optotechnik GmbH. Goettingen, Germany

[3.14] Gold mesh grid, 80 wire/inch, 0.01152 spacing, 0.00098 " diameter, $85.0 \%$ transmission, from Precision E-forming LLC, Cortland, NY 
CHAPTER 7: BIBLIOGRAPHY

[3.15] Aerodag aerosol graphite coating, from Acheson Industries

[3.16] T.J. Gay, et al., Rev. Sci. Instrum. 63, 114 (1992)

[3.17] D.J. Huang, et al., Rev. Sci. Instrum. 73, 3778 (2002)

[3.18] F.B. Dunning, et al., Rev. Sci. Instrum. 58, 1706 (1987)

[3.19] F.C. Tang, et al. Rev. Sci. Instrum. 59, 504 (1988)

[3.20] G.C. Burnett, T.J. Monroe, and F.B. Dunning, Rev. Sci. Instrum. 65, 1893 (1994)

[3.21] D.D. Neufeld, H. Aliabadi, and F.B. Dunning, Rev. Sci. Instrum. 78, 025107 (2007)

[3.22] J. Kessler, Polarized Electrons 2nd ed. (Springer-Verlag, Berlin, Germany 1985) p. 242,243

[3.23] Biased with two Bertan 375x NIM crate-mounted high voltage supply

[3.24] Ortec VT120a preamplifier

[3.25] Phillips 6930 discriminator

[3.26] J. Hansknecht, et al. AIP Conf. Proc.1149, 1143 (2009)

[3.27] C.K. Sinclair, et al., Phys. Rev. ST Accel. Beams 10, 023501 (2007)

[3.28] M. Baylac, et al., Phys. Rev. ST Accel. Beams 8, 123501 (2005)

[3.29] C. Leemann, D. Douglas, and G. Krafft, Annu. Rev. Nucl. Part. Sci. 51, 413 (2001)

[3.30] T. Maruyama, et al., Appl. Phys. Lett. 85, 2640 (2004)

[3.31] J.M. Grames, et al., Phys. Rev. ST Accel. Beams 7, 042802 (2004)

[3.32] GaAs epi-ready single crystal, 100 orientation, $\mathrm{Zn}$ doped $5 \times 10^{18}$ carrier concentration from AXT Corporation, Fremont, CA

[3.33] T. Maruyama, et al., Phys. Rev. B 46, 4261 (1992)

[3.34] M. Uhrig, et al., Rev. Sci. Instrum. 60, 872 (1989)

[3.35] D.P. Pappas and H. Hopster, Rev. Sci. Instrum. 60, 3068 (1989)

[3.36] S. Qiao, et al., Rev. Sci. Instrum. 68, 4390 (1997)

[3.37] V.N. Petrov, M.S. Galaktionov, and A.S. Kamochkin, Rev. Sci. Instrum. 72, 3728 (2001)

[3.38] F. Ciccacci, S. De Rossi, and D.M. Campbell, Rev. Sci. Instrum. 66, 4161 (1995) 
CHAPTER 7: BIBLIOGRAPHY

[4.1] H. Montgomery, J. Phys.: Conf. Ser. 299, 011001 (2011)

[4.2] C. Y. Prescott et al., Phys. Lett. B 77, 347-52 (1978)

[4.3] D. T. Pierce and F. Meier, Phys. Rev. B 13, 5484-5500 (1976)

[4.4] J. Kessler Polarized Electrons (Springer, New York, $19852^{\text {nd }}$ edition)

[4.5] T. Maruyama et al., Phys. Rev. Lett. 66, 2376 (1991)

[4.6] T. Nakanishi et al., Physics Lett. A 158, 345-349, (1991)

[4.7] T. Maruyama et al., Phys. Rev. B 46, 4261 (1992)

[4.8] T. Nakanishi et al., AIP Conf. Proc. 421, 300-10 (1998)

[4.9] J. Jian et al., Appl. Phys. Lett. 85, 2640 (2004)

[4.10] H. Aoyagi et al., Phys. Lett. A 167, 415 (1992)

[4.11] A. Yariv Optical Electronics (Holt, Rinehart and Winston, New York, 1985)

[4.12] T. Matsuyama et al. in Proceedings of the $4^{\text {th }}$ Pacific Rim Conf. on Lasers and Electro-Optics $164-52001$

[4.13] T. Matsuyama et al., Jpn. J. Appl. Phys. 40, 555-7 (2001)

[4.14] R. D. R. Bhat. et al., Phys. Rev. B 71, 035209 (2005)

[4.15] M. I. Miah, J. Phys. Chem. B 113, 6800-2 (2009)

[4.16] S. Krishnamurthy et al., J. Appl. Phys. 109, 033102 (2011)

[4.17] P. Yu and M. Cardona. Fundamentals of Semiconductors: Physics and Materials Properties (Springer, Berlin 2005) Section 2.6

[4.18] R. Winkler Spin-Orbit Coupling Effects in Two-Dimensional Electron and Hole Systems (Springer-Verlag, Berlin, 2003)

[4.19] D.T. Pierce and F. Meier, Appl. Phys. Lett. 101, 23301 (2012)

[4.20] K. Seeger, Semiconductor Physics, (Springer, Berlin 2004), Ch.5

[4.21] J. L. McCarter et al., Nucl. Instrum.and Meth. A 618, 30-6 (2010)

[4.22] H. M. Al-Khateeb et al., Rev. Sci. Instrum. 70, 3882 (1999) 
CHAPTER 7: BIBLIOGRAPHY

[4.23] T. G. Anderson et al., Rev. Sci. Instrum. 72, 2923 (2001)

[4.24] J. Hansknecht and M. Poelker, PRSTAB 9, 063501 (2006)

[4.25] C. K. Sinclair et al., PRSTAB 10, 023501 (2007)

[4.26] M. Woods et al., J. Appl. Phys. 73, 8531-35 (1993)

[4.27] C. K. Sinclair, Nucl. Instrum. and Meth. A 557, 69-74 (2006)

[4.28] J. Nelson, The Physics of Solar Cells (Properties of Semiconductor Materials) (Imperial College Press 2003) p.202

[4.29] H.C. Casey, Jr., D.D. Sell, and K.W. Wecht, J. Appl. Phys. 46, 250 (1975)

[4.30] G. L. Bir, A. G. Aronov, and G. E. Pikus, Sov. Phys. JETP 42, 705 (1976)

[4.31] M. Erbudak and B. Reihl. Appl. Phys. Lett. 33, 584 (1978)

[4.32] P. J. Saez, Ph.D. thesis, Stanford University, 1997

[4.33] R.I. Dzhioev, et al., Phys. of the Sol. State 45, 2255 (2003)

[5.1] I.V. Bazarov, et al., Appl. Phys. 103, 054901 (2008)

[5.2] D. H. Dowell, S.Z. Behtel, and K.D. Friddell, Nucl. Instrum. Methods A 356, 167 (1995)

[5.3] T. Vecchione, et al., Appl. Phys. Lett. 99, 034103 (2011)

[5.4] L. Cultrera, et al., Proceedings of IPAC2012, 2137 (2012)

[5.5] I.V. Bazarov, et al. Appl. Phys. Lett. 98, 224101 (2011)

[5.6] K. Aulenbacher, et al. Nucl. Instrum. Methods A 391, 498 (1997)

[5.7] G.D. Cates, et al. Nucl. Instrum. Methods A 278, 293 (1989)

[5.8] M.J.J. van den Putte, et al., AIP Conf. Proc. 421, 260 (1997)

[5.9] W. Hillert, M. Gowin, and B. Neff, AIP Conf. Proc. 570, 961 (2001)

[5.10] C. Hernandez-Garcia, et al., AIP Conf. Proc. 1149, 1071 (2009)

[5.11] L. Cultrea, et al., Phys. Rev. STAB 14, 120101 (2011)

[5.12] B. Dunham, et al., IPAC 2012 Conf. Proc. MOOAA01, ISBN 978-3-95450-115-1

[5.13] J. Grames, et al., Phys. Rev. STAB 14, 043501 (2011) 
CHAPTER 7: BIBLIOGRAPHY

[5.14] B.M. Dunham, Ph.D thesis, University of Illinois at Urbana-Champaign (1993)

[5.15] D.C. Carey, The Optics of Charged Particle Beams. (Harwood Academic Publishers, 1987)

[5.16] M.C. Ross, et al., SLAC Report No. 4278 (1987)

[5.17] R.H. Helm, SLAC Report No. 4, (1962)

[5.18] H. Fischer, et al., Proceedings of the Workshop on Photocathodes for Polarized Electron Sources for Accelerators, SLAC Report No. 432 (1994) p.249

[5.19] C. K. Sinclair, et al., Phys. Rev. STAB 10, 023501 (2007)

[5.20] C.K. Sinclair, Proceedings of PAC1999, (1999)

[5.21] K. Aulenbacher, et al., Proceedings of the Workshop on Photocathodes for Polarized Electron Sources for Accelerators, SLAC Report No. 432 (1994) p.1

[5.22] J. Grames, et al., Proceedings of PAC2005, (2005) p. 2875

[5.23] Alvasources Alkali Metal Dispenser, Cs or K, from Alvatec

[5.24] J. Smedley, T. Rao, and E. Wang, AIP Conf. Proc. 1149, 1062 (2009)

[5.25] P.A. Adderley, et al., Proceedings of PAC2011, (2011)

[5.26] M. BastaniNejad, et al., Proceedings of PAC2011, (2011)

[5.27] SAES WP1250-ST707 NEG Pump, from SAES Getters

[5.28] T. Vechhione, et al., Proceedings of IPAC2012, 655 (2012)

[5.29] S. Karkare and I. Bazarov, Appl. Phys. Lett. 98, 094104 (2011)

[5.30] M. Cardona and L. Ley, Photoemission in Solids I. General Principles, Topics in Applied Physics Vol. 26 (Springer, Berlin, 1978), Chap. 1

[5.31] D. Motta and S. Schönert, Nucl. Instrum. Methods A 539, 217 (2005)

[5.32] B. Karlsson and C. G. Ribbing, J. Appl. Phys. 53, 6340 (1982)

[5.33] P. Dolizy and F. Groliere, J. Phys. D: Appl. Phys. 19 (1986) 687 